\title{
Secure Information Flow for Concurrent Programs Under Total Store Order Supplemental technical material
}

\author{
Jeffrey A. Vaughan Todd Millstein \\ UCLA Compter Science Department \\ Technical Report \#120007 \\ May 9, 2012
}

\begin{abstract}
Modern multicore hardware and multithreaded programming languages expose weak memory models to programmers, which relax the intuitive sequential consistency (SC) memory model in order to support a variety of hardware and compiler optimizations. However, to our knowledge all prior work on secure information flow in a concurrent setting has assumed SC semantics. This paper investigates the impact of the Total Store Order (TSO) memory model, which is used by Intel x86 and Sun SPARC processors, on secure information flow, focusing on the natural security condition known as possibilistic noninterference. We show that possibilistic noninterference under SC and TSO are incomparable notions; neither property implies the other one. We define a simple type system for possibilistic noninterference under SC and demonstrate that it is not sound under TSO. We then provide two variants of this type system that are sound under TSO: one that requires only a small change to the original type system but is overly restrictive, and another that incorporates a form of flow sensitivity to safely retain desired expressiveness. Finally, we show that the original type system is in fact sound under TSO for programs that are free of data races. This report is a companion to "Secure Information Flow for Concurrent Programs Under Total Store Order" (Vaughan and Millstein, 2012).
\end{abstract}

\section{Overview}

This document presents the proofs and full definitions for "Secure Information Flow for Concurrent Programs Under Total Store Order" (Vaughan and Millstein, 2012).

The conference paper's type system only accepts well-structured source programs. For the proofs, it is convenient to present an expanded type system that accepts intermediate program states and to track the sourceness and well-structuredness properties separately. Additionally, results are numbered differently in the two documents. The chart below shows how these correspond.

\begin{tabular}{|l|l|}
\hline Conference paper & This document \\
\hline Theorem 3 & Corollary 10 \\
Theorem 4 & Corollary 3 \\
Theorem 6 & Corollary 7 \\
Theorem 7 & Lemma 111 \\
Theorem 8 & Lemma 112 \\
Theorem 15 & Theorem 2 \\
\hline
\end{tabular}

\section{Language definition}

\subsection{Language syntax and main semantic sets}

This syntax of the concurrent imperative language, and definitions of the main semantic objects are as follows. 


\begin{tabular}{|c|c|c|c|c|}
\hline Local variables & $x, y, z$ & $\epsilon$ & LocalVar & \\
\hline Shared variables & $X, Y, Z$ & $\in$ & HeapVar & \\
\hline Variables, Var & $v$ & $::=$ & LocalVar $\cup$ HeapVar & \\
\hline Locks & $\ell$ & $\in$ & Lock & A finite set \\
\hline Integer literals & $i$ & $\in$ & $\mathbb{Z}$ & \\
\hline Boolean literals & $\beta$ & $\in$ & $\mathbb{B}=\{$ true, false $\}$ & \\
\hline Arithmetic Exp. & $a$ & $\stackrel{:=}{\mid}$ & $\begin{array}{l}x \\
i \\
a \oplus a|(b ? a: a)| \ldots\end{array}$ & $\begin{array}{l}\text { Local var } \\
\text { Integer literal } \\
\text { Arithmetic ops. }\end{array}$ \\
\hline Boolean Exp. & $b$ & $\stackrel{:=}{\mid}$ & $\begin{array}{l}\beta \\
\text { isZero } a \\
b \otimes b\end{array}$ & $\begin{array}{l}\text { Boolean literal } \\
\text { Zero test } \\
\text { Boolean ops. }\end{array}$ \\
\hline Command & $c, d$ & $\begin{array}{l}:= \\
\mid \\
\mid \\
\mid \\
\mid \\
\mid \\
\mid\end{array}$ & $\begin{array}{l}x:=X \\
X:=x \\
x:=a \\
\text { sync } \ell \text { do } c \\
\text { holding } \ell \text { do } c \\
\text { fence } \\
\text { fork } c \\
c_{1} ; c_{2} \mid \text { if } b \text { do } c_{1} \text { else } c_{2} \\
\text { while } b \text { do } c \mid \text { skip }\end{array}$ & $\begin{array}{l}\text { Load } \\
\text { Store } \\
\text { Expression evaluation } \\
\text { Lock acquire } \\
\text { Lock held (forbidden in source programs) } \\
\text { Memory barrier } \\
\text { Thread creation } \\
\text { While language commands }\end{array}$ \\
\hline Write buffer, WriteBuf & $W$ & $\stackrel{:=}{\mid}$ & $\begin{array}{l}n i l \\
(X:=i):: W\end{array}$ & $\begin{array}{l}\text { Empty } \\
\text { Write pending (ready to commit at head, } \\
\text { newer writes in tail) }\end{array}$ \\
\hline Local state & $L$ & $\epsilon$ & $\begin{array}{l}(\text { LocalVar } \rightarrow \mathbb{Z}) \times \mathcal{P}(\text { Lock }) \\
\quad \times \text { WriteBuff }\end{array}$ & \\
\hline Thread IDs & $\iota$ & $\epsilon$ & TID & A finite set \\
\hline Thread & $s, t$ & $::=$ & $\langle L, c\rangle_{\iota}$ & \\
\hline Process soup & $P, Q$ & $\stackrel{:=}{\mid}$ & $\begin{array}{l}\mathcal{o} \\
t \| P\end{array}$ & $\begin{array}{l}\text { Nil process } \\
\text { Parallel composition }\end{array}$ \\
\hline Global state & $G, H$ & $\epsilon$ & $($ HeapVar $\rightarrow \mathbb{Z}) \times \mathcal{P}($ Lock $)$ & \\
\hline Configuration, Config & $\chi$ & $::=$ & $(G, P)$ & \\
\hline Operation & $o p$ & $::=$ & eval $\mid$ commit & \\
\hline Action, Action & $\alpha$ & $::=$ & $o p(i)$ & \\
\hline Action Set & $A$ & $\in$ & $\mathcal{P}($ Action $)$ & \\
\hline
\end{tabular}




\subsection{Notation}

Suppose local state $L=(M, \lambda, W)$. We write L.mem, L.locks, and $L$.wb for $M, \lambda$, and $W$ respectively. If $x \in$ LocalVar we write $L[x \mapsto i]$ for $(M[x \mapsto i], \lambda, W)$ and $L(x)$ for $M(x)$. Likewise we use $L \cup \kappa$ and $L \cap \kappa$ and $L \backslash \kappa$ and $\ell \in L$ for $(M, \lambda \cup \kappa)$ and $(M, \lambda \cap \kappa)$ and $(M, \lambda \backslash \kappa)$ and $\ell \in \lambda$. Finally we use $L+(X:=i)$ for $(M, \lambda, W+(X:=i):: n i l)$ and $(X:=i):: L$ for $(M, \lambda,(X:=i):: W)$.

Symbol $L_{\oslash}$ represents the "empty" local state $((\lambda x .0), \emptyset, n i l)$.

Suppose $G=(S, \lambda)$. We write G.mem for $S$ and $G$.locks for $\lambda$.

Suppose $t=\langle L, c\rangle$. Then t.cmd, t.ls, t.locks, t.wb, and t.mem mean $c, L, L . l o c k s, L . w b$, and L.mem respectively.

Metavariable $S$, for Store, ranges over global heaps and metavariable $M$, for Memory, ranges over local memories.

Thread ids are only important for the argument in Section 4 and are often suppressed to avoid clutter.

We will sometimes write singleton process $t \|$ o simply as $t$. Additionally we define $Q+P$ to be the process soup formed by appending $Q$ and $P$; that is $\mathrm{o}+P=P$ and $(t \| Q)+P=t \|(Q+P)$. We abuse notation and write $t \in P$ when $P \equiv t \| Q$ for some $Q$ as well as $P \| Q$ for $P+Q$. Finally we write nonempty $P$ when has form $t \| P_{0}$ and for such a nonempty process soup, $h d(P)$ is $t$.

\subsection{Single Step Operations}

$$
(S ; W)[X] \Downarrow i
$$

$$
\overline{(S ; W+(X:=i))[X] \Downarrow i} \quad \frac{X \neq Y \quad(S ; W)[X] \Downarrow i}{\left(S ; W+\left(Y:=i_{0}\right)\right)[X] \Downarrow i} \quad \overline{(S ; n i l)[X] \Downarrow S(X)}
$$

\section{$L[a] \Downarrow i$}

$$
\begin{gathered}
\overline{L[x] \Downarrow L(x)} \quad \frac{L\left[a_{1}\right] \Downarrow i_{1} \quad L\left[a_{2}\right] \Downarrow i_{2} \quad i=i_{1} \llbracket \oplus \rrbracket i_{2}}{L[i] \Downarrow i} \quad \frac{L[b] \Downarrow \text { true } \quad L\left[a_{1}\right] \Downarrow i}{L\left[\left(b ? a_{1}: a_{2}\right)\right] \Downarrow i} \\
\frac{L[b] \Downarrow \text { false } \quad L\left[a_{2}\right] \Downarrow i}{L\left[\left(b ? a_{1}: a_{2}\right)\right] \Downarrow i}
\end{gathered}
$$

\section{$L[b] \Downarrow \beta$}

$\frac{L[a] \Downarrow 0}{L[\beta] \Downarrow \beta} \quad \frac{L[a] \Downarrow i \quad i \neq 0}{L[\text { isZero } a] \Downarrow \text { true }} \quad \frac{L\left[b_{1}\right] \Downarrow \beta_{1}}{L[\text { isZero } a] \Downarrow \text { false }} \quad \frac{L\left[b_{2}\right] \Downarrow \beta_{2} \quad \beta=\beta_{1} \llbracket \oslash \rrbracket \beta_{2}}{L\left[b_{1} \oslash b_{2}\right] \Downarrow \beta}$

\footnotetext{
$(G, t) \longrightarrow^{\text {commit }}\left(G^{\prime}, P\right)$
}

$$
\overline{(G,\langle(X:=i):: L, c\rangle) \longrightarrow^{\text {commit }}(G[X \mapsto i],\langle L, c\rangle)}
$$




$$
(G, t) \longrightarrow^{\text {eval }}\left(G^{\prime}, P\right)
$$

$$
\begin{aligned}
& {\overline{(G,\langle L, X:=x\rangle) \longrightarrow^{\text {eval }}(G,\langle L+(X:=L(x)), \text { skip }\rangle)}}^{\text {EC-STore }} \\
& \frac{(\text { G.mem } ; \text { L.wb })[X] \Downarrow i}{(G,\langle L, x:=X\rangle) \longrightarrow^{\text {eval }}(G,\langle L[x \mapsto i], \text { skip }\rangle)} \text { EC-LOAD } \\
& \frac{L[a] \Downarrow i}{(G,\langle L, x:=a\rangle) \longrightarrow^{e v a l}(G,\langle L[x \mapsto i], \mathbf{s k i p}\rangle)} \text { EC-EvALEXP } \\
& \frac{\ell \in G \quad L . w b=n i l}{(G,\langle L, \text { sync } \ell \text { do } c\rangle) \longrightarrow^{e v a l}(G \backslash\{\ell\},\langle L \cup\{\ell\}, \text { holding } \ell \text { do } c\rangle)} \text { EC-SyncAcQuire } \\
& \frac{\ell \in L}{(G,\langle L, \text { sync } \ell \text { do } c\rangle) \longrightarrow^{\text {eval }}(G,\langle L, \text { fence } ;(c ; \text { fence })\rangle)} \text { EC-SyncReEnter } \\
& \frac{\ell \in L \quad\left(G,\langle L, c\rangle_{\iota}\right) \longrightarrow^{e v a l}\left(G^{\prime},\left\langle L^{\prime}, c^{\prime}\right\rangle_{\iota} \| P\right)}{\left(G,\langle L, \text { holding } \ell \text { do } c\rangle_{\iota}\right) \longrightarrow^{\text {eval }}\left(G^{\prime},\left\langle L^{\prime}, \text { holding } \ell \text { do } c^{\prime}\right\rangle_{\iota} \| P\right)} \text { EC-HoldSteP } \\
& \frac{\ell \in L \quad L . w b=n i l}{(G,\langle L, \text { holding } \ell \text { do skip }\rangle) \longrightarrow^{\text {eval }}(G \cup\{\ell\},\langle L \backslash\{\ell\}, \text { skip }\rangle)} \text { EC-HOLdRELEASE } \\
& \frac{L . w b=n i l}{(G,\langle L, \text { fence }\rangle) \longrightarrow^{e v a l}(G,\langle L, \text { skip }\rangle)} \text { EC-FENCE } \\
& \frac{L . w b=\text { nil } \quad \iota^{\prime} \text { fresh }}{\left(G,\langle L, \text { fork } c\rangle_{\iota}\right) \longrightarrow^{\text {eval }}\left(G,\langle L, \text { skip }\rangle_{\iota} \|\left\langle L_{\oslash}, c\right\rangle_{\iota^{\prime}}\right)} \text { EC-FORK } \\
& \frac{\left(G,\left\langle L, c_{1}\right\rangle_{\iota}\right) \longrightarrow^{e v a l}\left(G^{\prime},\left\langle L^{\prime}, c_{1}^{\prime}\right\rangle_{\iota} \| P\right)}{\left(G,\left\langle L, c_{1} ; c_{2}\right\rangle_{\iota}\right) \longrightarrow^{e v a l}\left(G^{\prime},\left\langle L^{\prime}, c_{1}^{\prime} ; c_{2}\right\rangle_{\iota} \| P\right)} \text { EC-SeQStruCt } \\
& \overline{\left(G,\langle L, \text { skip } ; c\rangle_{\iota}\right) \longrightarrow^{\text {eval }}\left(G,\langle L, c\rangle_{\iota}\right)} \text { EC-SEQSKIP } \\
& \frac{L[b] \Downarrow \text { true }}{\left(G,\left\langle L, \text { if } b \text { do } c_{1} \text { else } c_{2}\right\rangle\right) \longrightarrow^{\text {eval }}\left(G,\left\langle L, c_{1}\right\rangle\right)} \text { EC-IFTrue } \\
& \frac{L[b] \Downarrow \text { false }}{\left(G,\left\langle L, \text { if } b \text { do } c_{1} \text { else } c_{2}\right\rangle\right) \longrightarrow{ }^{\text {eval }}\left(G,\left\langle L, c_{2}\right\rangle\right)} \text { EC-IFFALSE } \\
& \frac{L[b] \Downarrow \text { true }}{\left(G,\langle L, \text { while } b \text { do } c\rangle_{\iota}\right) \longrightarrow^{\text {eval }}\left(G,\langle L, c ; \text { while } b \text { do } c\rangle_{\iota}\right)} \text { EC-WhILETrue } \\
& \frac{L[b] \Downarrow \text { false }}{\left(G,\langle L, \text { while } b \text { do } c\rangle_{\iota}\right) \longrightarrow^{\text {eval }}\left(G,\langle L, \text { skip }\rangle_{\iota}\right)} \text { EC-WhILEFALSE } \quad \frac{L . w b=n i l \quad L . l o c k s=\emptyset}{(G,\langle L, \text { skip }\rangle) \longrightarrow^{\text {eval }}(G, \mathbf{o})} \text { EC-REAP }
\end{aligned}
$$




\subsection{Possibilistic evaluation}

$$
o p(i) \in \operatorname{Ready}\left(G, t_{1} \cdots t_{i} \cdots t_{n}\right) \text { iff exists } \chi \text { such that }\left(G, t_{i}\right) \longrightarrow^{o p} \chi
$$

$$
\operatorname{ReadySC}(\chi)= \begin{cases}\text { commits } & \text { commits } \neq \emptyset \\ \operatorname{Ready}(\chi) & \text { otherwise }\end{cases}
$$

$$
\text { where commits }=\{\operatorname{commit}(i) \mid \operatorname{commit}(i) \in \operatorname{Ready}(\chi)\}
$$

$$
(G, P) \Longrightarrow{ }^{\mathrm{sc}}\left(G^{\prime}, P^{\prime}\right)
$$

$$
\begin{gathered}
P=t_{1} \ldots t_{i-1}\left\|t_{i}\right\| t_{i+1} \ldots t_{n} \quad \begin{array}{c}
o p(i) \in \operatorname{ReadySC}(\chi) \\
\left(G, t_{i}\right) \longrightarrow^{o p}\left(G^{\prime}, Q\right)
\end{array} \quad P^{\prime}=t_{1} \ldots t_{i-1}\|Q\| t_{i+1} \ldots t_{n} \\
\hline(G, P) \Longrightarrow^{\mathrm{sc}}\left(G^{\prime}, P^{\prime}\right)
\end{gathered}
$$

$(G, P) \Longrightarrow^{\text {tso }}\left(G^{\prime}, P^{\prime}\right)$

$$
\begin{array}{ll}
P=t_{1} \ldots t_{i-1}\left\|t_{i}\right\| t_{i+1} \ldots t_{n} \quad\left(G, t_{i}\right) \longrightarrow^{o p}\left(G^{\prime}, Q\right) \quad P^{\prime}=t_{1} \ldots t_{i-1}\|Q\| t_{i+1} \ldots t_{n} \\
(G, P) \Longrightarrow^{\text {tso }}\left(G^{\prime}, P^{\prime}\right)
\end{array}
$$

Define $\Longrightarrow^{\mathrm{mm} *}$ as the reflexive, transitive closure of the $\Longrightarrow^{\mathrm{mm}}$ relation where $\mathrm{mm}$ is either sc or tso.

\section{$3 \quad$ A simple type system for possibilistic flows}

This is a minimal delta from (Smith and Volpano, 1998). Typing uses the following syntactic classes.

$$
\begin{array}{llll}
\text { Syntactic Security Level } & \tau & :=\text { low } \mid \text { high } \\
\text { Static Security Context } & \Gamma & :=\text { HeapVar } \cup \text { LocalVar } \cup \text { Lock } \rightarrow \tau
\end{array}
$$

We define lattice operators for syntactic security levels: least upper bound $\sqcup$, greatest lower bound $\sqcap$, and partial order $\sqsubseteq$. These respect the ordering low $\sqsubseteq$ high.

\subsection{Types and basic properties}

$$
\Gamma \vdash a: \tau
$$$$
\frac{\Gamma(x) \sqsubseteq \tau}{\Gamma \vdash x: \tau} \quad \frac{\Gamma \vdash a_{1}: \tau \quad \Gamma \vdash a_{2}: \tau}{\Gamma \vdash a_{1} \oplus a_{2}: \tau} \quad \frac{\Gamma \vdash b: \tau \quad \Gamma \vdash a_{1}: \tau \quad \Gamma \vdash a_{2}: \tau}{\Gamma \vdash\left(b ? a_{1}: a_{2}\right): \tau}
$$

$\Gamma \vdash b: \tau$

$$
\overline{\Gamma \vdash \beta: \tau} \quad \frac{\Gamma \vdash a: \tau}{\Gamma \vdash \text { isZero } a: \tau} \quad \frac{\Gamma \vdash b_{1}: \tau \quad \Gamma \vdash b_{2}: \tau}{\Gamma \vdash b_{1} \otimes b_{2}: \tau}
$$




$$
p c ; \Gamma \vdash^{\text {tso }} c
$$$$
\frac{p c \sqcup \Gamma(Y) \sqsubseteq \Gamma(x)}{p c ; \Gamma \vdash^{\text {tso }} x:=Y} \text { TSO-LoAD } \quad \frac{p c \sqcup \Gamma(y) \sqsubseteq \Gamma(X)}{p c ; \Gamma \vdash^{\text {tso }} X:=y} \text { Tso-Store } \quad \frac{\Gamma \vdash a: \tau \quad p c \sqcup \tau \sqsubseteq \Gamma(x)}{p c ; \Gamma \vdash^{\text {tso }} x:=a} \text { TSO-EvAL }
$$

$$
\frac{\Gamma(\ell) ; \Gamma \vdash^{\text {tso }} c}{l o w ; \Gamma \vdash^{\text {tso }} \text { sync } \ell \text { do } c} \text { TSO-SynC } \quad \frac{\Gamma(\ell) ; \Gamma \vdash^{\text {tso }} c}{l o w ; \Gamma \vdash^{\text {tso }} \text { holding } \ell \text { do } c} \text { TsO-HoLD }
$$

$$
\frac{p c ; \Gamma \vdash^{\mathrm{tso}} c}{l o w ; \Gamma \vdash^{\mathrm{tso}} \text { fence }} \text { TSO-Fence } \quad \frac{p c ; \Gamma \vdash^{\mathrm{tso}} c_{1} p c ; \Gamma \vdash^{\mathrm{tso}} c_{2}}{p c ; \Gamma \vdash^{\mathrm{tso}} c_{1} ; c_{2}} \text { TSO-SEQ }
$$

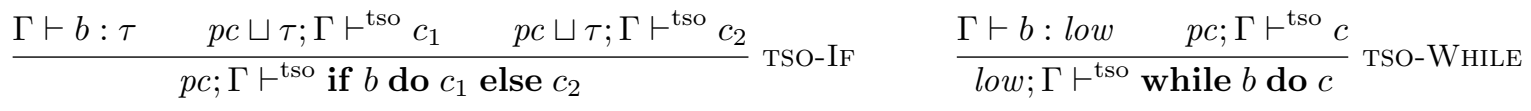

$$
\overline{p c ; \Gamma \vdash^{\mathrm{tso}} \text { skip }} \text { TSO-SKIP }
$$

$p c ; \Gamma \vdash^{\text {tso }} \lambda$

$$
\overline{p c ; \Gamma \vdash^{\mathrm{tso}}\{\}} \quad \overline{l o w ; \Gamma \vdash^{\mathrm{tso}} \lambda}
$$

$p c ; \Gamma \vdash^{\text {tso }} W$

$$
\overline{p c ; \Gamma \vdash^{\mathrm{tso}} n i l} \quad \frac{p c \sqsubseteq \Gamma(X) \quad p c ; \Gamma \vdash^{\mathrm{tso}} W}{p c ; \Gamma \vdash^{\mathrm{tso}}(X:=i):: W}
$$

$p c ; \Gamma \vdash^{\text {tso }} t$

$$
\frac{p c ; \Gamma \vdash^{\text {tso }} \lambda \quad p c ; \Gamma \vdash^{\text {tso }} W \quad p c ; \Gamma \vdash^{\text {tso }} c}{p c ; \Gamma \vdash^{\text {tso }}\langle(M, \lambda, W), c\rangle_{\iota}}
$$

$\overline{p c} ; \Gamma \vdash^{\text {tso }} P$

$$
\frac{p c ; \Gamma \vdash^{\text {tso }} t \quad \overline{p c} ; \Gamma \vdash^{\text {tso }} P}{p c, \overline{p c} ; \Gamma \vdash^{\text {tso }} t \| P}
$$

$p c \sqsubseteq \overline{p c}$

$$
\overline{p c \sqsubseteq .} \quad \frac{p c \sqsubseteq p c_{0} \quad p c \sqsubseteq \overline{p c}}{p c \sqsubseteq p c_{0}, \overline{p c}}
$$




\subsection{Properties of syntax and evaluation}

Definition 1 (size).

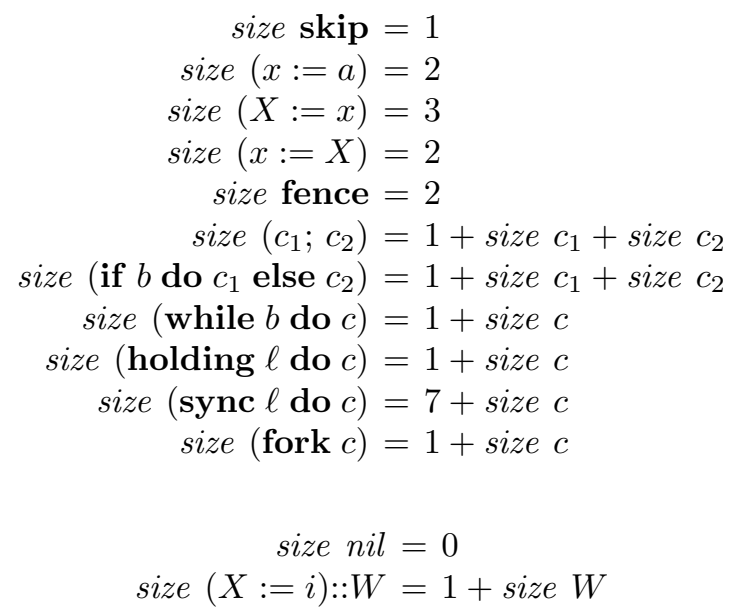

\subsection{Evaluation contexts}

$$
\begin{array}{ll}
\text { Command Context } & \mathcal{C}::=[\cdot]|\mathcal{C} ; c| \text { holding } \ell \text { do } \mathcal{C} \\
\text { Evaluation Context } & \mathcal{E}=(\lambda, \mathcal{C})
\end{array}
$$

Notation $\mathcal{C}[c]$ has the usual meaning and when $\mathcal{E}=(\lambda, \mathcal{C})$ write $\mathcal{E}\left[W \mid\langle L, c\rangle_{\iota}\right]$ for $\langle W+L \cup \lambda, \mathcal{C}[c]\rangle_{\iota}$. Notation $\mathcal{E}_{\emptyset}$ means $([\cdot], \emptyset)$.

We define $\mathcal{C}$.locks as follows:

$$
\begin{aligned}
{[\cdot] \text { locks } } & =\emptyset \\
(\mathcal{C} ; c) . \text { locks } & =\mathcal{C} \text {.locks } \\
\text { (holding } \ell \text { do } \mathcal{C}) . \text { locks } & =\{\ell\} \cup \mathcal{C} \text {.locks. }
\end{aligned}
$$

Definition 2 (Active evaluation context). Evaluation context $(\lambda, \mathcal{C})$ is active, written active $(\lambda, \mathcal{C})$, when C.locks $\subseteq \lambda$.

Lemma 1. If $(G, \mathcal{E}[W \mid t]) \longrightarrow^{\text {eval }}\left(G^{\prime}, P^{\prime}\right)$ then active $\mathcal{E}$.

Proof. Assume for a contradiction that $\mathcal{E}=(\lambda, \mathcal{C})$ is not active. Then there is a stuck holding command in $\mathcal{C}$ which contracts the assumption that $\mathcal{E}[W \mid t]$ takes an eval-step.

Definition 3 (canEval). If there exist $G, G^{\prime}$, and $P^{\prime}$ such that $(G, t) \longrightarrow{ }^{\text {eval }}\left(G^{\prime}, P^{\prime}\right)$, then we say canEval $t$.

Lemma 2. Suppose canEval $(\mathcal{E}[t])$. Then there exists $\mathcal{E}_{0}$ such that active $\mathcal{E}_{0}$ and $\mathcal{E}_{0}[t]=\mathcal{E}[t]$.

Proof. Let $\mathcal{E}=(\lambda, \mathcal{C})$ and define $\mathcal{E}_{0}=(\lambda \cup \mathcal{C}$.locks, $\mathcal{C})$. From canEval $\mathcal{E}[t]$ we know that for some $G$, $G^{\prime}$, and $P^{\prime}$ it is the case that $(G, t) \longrightarrow^{\text {eval }}\left(G^{\prime}, P^{\prime}\right)$. To show $\mathcal{E}_{0}[t]=\mathcal{E}[t]$ if suffices to show that $\mathcal{C}$.locks $\subseteq$ t.ls.locks, which follows from a simple induction on the $\longrightarrow{ }^{\text {eval }}$ relation.

Lemma 3. If $(G, P) \Longrightarrow^{\text {tso* }}\left(G^{\prime}, P^{\prime}\right)$, then $\left(G, P \| P_{0}\right) \Longrightarrow^{\text {tso* }}\left(G^{\prime}, P^{\prime} \| P_{0}\right)$.

Definition 4 (hasEmptyWBs $(P))$. We say $P$ has empty write buffers, written hasEmptyWBs $(P)$, if for all $t$ such that $P=P_{1}\|t\| P_{2}$, it is the case that $t . w b=$ nil.

Lemma 4. If $(G, P) \Longrightarrow^{\mathrm{sc} *}\left(G^{\prime}, P^{\prime}\right)$, and hasEmptyWBs $\left(P_{0}\right)$ then $\left(G, P \| P_{0}\right) \Longrightarrow^{\mathrm{sc} *}\left(G^{\prime}, P^{\prime} \| P_{0}\right)$.

Definition 5. Source programs, and well structured contexts and commands

$\operatorname{src} c$

$\begin{array}{cccccc}\frac{\operatorname{src}(x:=X)}{\operatorname{src}(X:=x)} & \frac{\operatorname{src} c}{\operatorname{src}(x:=X)} & \frac{\operatorname{src}(\text { sync } \ell \text { do } c)}{\operatorname{src}} & \frac{\operatorname{src} c}{\operatorname{sence} \text { fork } c} \\ \frac{\operatorname{src} c_{1} \quad \operatorname{src} c_{2}}{\operatorname{src} c_{1} ; c_{2}} & \frac{\operatorname{src} c_{1} \quad \operatorname{src} c_{2}}{\operatorname{src} \text { if } b \text { do } c_{1} \text { else } c_{2}} & \frac{\operatorname{src} \text { while } b \text { do } c}{\operatorname{src} \text { skip }}\end{array}$


wellStruct $\mathcal{C}$

$\frac{\text { wellStruct }[\cdot]_{\text {wellStruct } \mathcal{C} \quad \text { src } c}^{\text {wellStruct } \mathcal{C} ; c}}{\frac{\text { wellStruct } \mathcal{C} \quad \ell \notin \mathcal{C} . \text { locks }}{\text { wellStruct holding } \ell \mathbf{d o} \mathcal{C}}}$

wellStruct $c$

$\frac{\text { wellStruct } \mathcal{C} \quad \text { src } c}{\text { wellStruct } \mathcal{C}[c]}$

wellStruct $t$

$$
\frac{\text { wellStruct } c}{\text { wellStruct }\langle L, c\rangle}
$$

wellStruct $P$

$$
\overline{\text { wellStruct } \mathrm{o}} \quad \frac{\text { wellStruct } t \quad \text { wellStruct } P}{\text { wellStruct } t \| P}
$$

There is one interesting difference between the abridged definitions give in the conference submission and the full versions in this this document. The type systems presented in the paper only apply to source commands. In this document we extend typing to include threads, locksets, write buffers, and non-source commands that occur during evaluation. Thus the premises of some theorems in this document contain additional hypotheses stating that $c$ is either a source command or is well structured, but these hypotheses are not needed in the submission as they are implied by typing.

Lemma 5. Suppose $(G, P) \Longrightarrow{ }^{\text {to* }}\left(G^{\prime}, P^{\prime}\right)$. If wellStruct $P$ then wellStruct $P^{\prime}$.

Proof. by nested induction on the length of the $\Longrightarrow{ }^{\text {tso* }}$ derivation then induction on each $\longrightarrow^{\text {eval }}$ derivation or trivial consideration of $\longrightarrow^{\text {commit }} \mathrm{s}$.

Lemma 6. Suppose wellStruct $c$ and $c=\mathcal{C}\left[c_{0}\right]$, then wellStruct $c_{0}$ and wellStruct $\mathcal{C}$.

Proof. by structural induction on $c$.

Definition 6 (c.locks).

$$
\begin{aligned}
\text { (holding } \ell \text { do } c) . \text { locks } & =\{\ell\} \cup \text { c.locks } \\
(\text { sync } \ell \text { do } c) . \text { locks } & =\{\ell\} \cup \text { c.locks } \\
\left(c_{1} ; c_{2}\right) . \text { locks } & =c_{1} . \text { locks } \cup c_{2} . \text { locks } \\
(\text { skip). locks } & =\emptyset
\end{aligned}
$$

Lemma 7. Suppose $\mathcal{E}=(\lambda$, holding $\ell$ do $[\cdot])$ and wellStruct $\mathcal{E}[t]$. If $(G, \mathcal{E}[t]) \longrightarrow{ }^{\text {eval }}\left(G^{\prime}, \mathcal{E}\left[t^{\prime}\right] \| P^{\prime}\right)$ then $\ell \in t^{\prime}$.

Proof. Use induction on the wellStruct derivation to show that $t$ does not contain redexes of the form holding $\ell$ do _, then continue by induction on the $\longrightarrow^{\text {eval }}$ derivation.

Lemma 8. Suppose wellStruct $c$ and c.locks $=\emptyset$. Then for any $\ell$ it is the case that wellStruct holding $\ell$ do $c$. 
Proof. by induction on the derivation of wellStruct $c$.

Lemma 9. Suppose $\left(G, t_{1}\left\|\ldots t_{n}\right\| \mathrm{o}\right) \Longrightarrow^{\mathrm{mm} *}\left(G^{\prime}, t_{1}^{\prime}\left\|\ldots t_{m}^{\prime}\right\| \mathrm{o}\right)$. Then

$$
\text { G.locks } \cup t_{1} \text {.ls.locks } \cup \ldots \cup t_{n} \text {.ls.locks }=G^{\prime} \text {.locks } \cup t_{1}^{\prime} \text {.ls.locks } \cup \ldots \cup t_{m}^{\prime} \text {.ls.locks. }
$$

Proof. by easy induction.

\subsection{Typing properties}

Definition 7 (tailOf $\left.\left(W_{0}, W\right)\right)$. Write buffer $W_{0}$ is the tail of $W$, written tailOf $\left(W_{0}, W\right)$, when $W=$ $(X:=i):: W_{0}$ for some $X$ and $i$.

Lemma 10 (Step preservation). Suppose $p c ; \Gamma \vdash^{\text {tso }} t$ and $(G, \mathcal{E}[W \mid t]) \longrightarrow^{o p}\left(G^{\prime}, \mathcal{E}\left[W^{\prime} \mid t^{\prime}\right] \| P^{\prime}\right)$. Further suppose either $W=W^{\prime}$, or both op $=$ commit and tailOf $\left(W^{\prime}, W\right)$. Then $p c ; \Gamma \vdash^{\text {tso }} t^{\prime}$ and $\overline{p c} ; \Gamma \vdash^{\text {tso }} P^{\prime}$ with $p c \sqsubseteq \overline{p c}$.

Proof. By induction on the typing relation.

Lemma 11 (Subtyping). Suppose $\tau_{2} \sqsubseteq \tau_{1}$. The following implications hold:

- $\Gamma \vdash a: \tau_{2}$ implies $\Gamma \vdash a: \tau_{1}$

- $\Gamma \vdash b: \tau_{2}$ implies $\Gamma \vdash b: \tau_{1}$

- $\tau_{1} ; \Gamma \vdash^{\text {tso }} c$ implies $\tau_{2} ; \Gamma \vdash^{\text {tso }} c$

- $\tau_{1} ; \Gamma \vdash^{\text {tso }} \lambda$ implies $\tau_{2} ; \Gamma \vdash^{\text {tso }} \lambda$

- $\tau_{1} ; \Gamma \vdash^{\text {tso }} W$ implies $\tau_{2} ; \Gamma \vdash^{\text {tso }} W$

- $\tau_{1} ; \Gamma \vdash^{\text {tso }} t$ implies $\tau_{2} ; \Gamma \vdash^{\text {tso }} t$

Proof. By induction.

Lemma 12 (Eval preservation). Suppose $\overline{p c} ; \Gamma \vdash^{\text {tso }} P$ where $p c \sqsubseteq \overline{p c}$. If $(G, P) \Longrightarrow \Longrightarrow^{m *}\left(G^{\prime}, P^{\prime}\right)$ then $\overline{p c}^{\prime} ; \Gamma \vdash^{\text {tso }} P^{\prime}$ where $p c \sqsubseteq \overline{p c}^{\prime}$.

Proof. by induction on the number of evaluation steps. The lemma holds trivially when there are zero steps. Instead suppose the trace contains $n+1$ steps. We have

$$
(G, P)=\left(G, P_{1}\|t\| P_{2}\right) \Longrightarrow^{m}\left(H, P_{1}\|Q\| P_{2}\right) \Longrightarrow^{m *}\left(G^{\prime}, P^{\prime}\right)
$$

where $(G, t) \longrightarrow^{o p}(H, Q)$. Using the induction hypothesis and the definition of $\vdash^{\text {tso }}$, it suffices to show that $\overline{p c}_{Q} ; \Gamma \vdash^{\text {tso }} Q$ for some $\overline{p c}_{Q}$ where $p c \sqsubseteq \overline{p c}_{Q}$. If $Q=\mathrm{o}$ this is immediate. If instead $Q=t^{\prime} \| Q_{0}$ then for $\mathcal{E}=(\emptyset,[\cdot])$ we have $(G, \mathcal{E}[$ nil $\mid t]) \longrightarrow^{o p}\left(H, \mathcal{E}\left[n i l \mid t^{\prime}\right] \| Q_{0}\right)$. Inverting the definition of $\vdash^{\text {tso }}$ yields $p c_{t} ; \Gamma \vdash^{\text {tso }} t$ where $p c \sqsubseteq p c_{t}$. By Lemma 10 , both $p c_{t} ; \Gamma \vdash^{\text {tso }} t^{\prime}$ and $\overline{p c}_{Q_{0}} ; \Gamma \vdash^{\text {tso }} Q_{0}$ where $p c_{t} \sqsubseteq \overline{p c}{ }_{Q_{0}}$. Conclude using the definition of $\vdash^{\text {tso }}$ and the transitivity of $\sqsubseteq$.

Lemma 13 (Total write-buffer typing). For all $W$, low; $\Gamma \vdash^{\text {tso }} W$.

Proof. By inspection. 


\subsection{Trace properties}

Definition 8 (Front-Reap-Freedom, Commit-Freedom and Simple Traces). Let $\mathcal{T}$ range over non-empty sequences of of configurations.

Write $\mathcal{T}::\left(G_{1}, P_{1}\right) \Longrightarrow^{\text {tso* }}\left(G_{n}, P_{n}\right)$ when $\mathcal{T}$ has form $\left(G_{1}, P_{1}\right),\left(G_{2}, P_{2}\right) \ldots\left(G_{n}, P_{n}\right)$ and for each $i \in\{1 \ldots n-1\}$ it is the case that $\left(G_{i}, P_{i}\right) \Longrightarrow{ }^{\text {tso }}\left(G_{i+1}, P_{i+1}\right)$.

$W e$ say FrontReapFree $\mathcal{T}$ when for each such $i$, there exist thread pools $P, Q, R$ and thread $t$ such that

$$
\begin{aligned}
P_{i} & =P\|t\| Q \\
P_{i+1} & =P\|R\| Q
\end{aligned}
$$

where either $P$ is non-empty or $\left(G_{i}, t\right) \longrightarrow^{o p}\left(G_{i+1}, R\right)$ by a rule other than EC-REAP.

We say FrontCommitFree $\mathcal{T}$ when for each $i$,

$$
\begin{aligned}
P_{i} & =P\|t\| Q \\
P_{i+1} & =P\|R\| Q
\end{aligned}
$$

either $P$ is non-empty or $\left(G_{i}, t\right) \longrightarrow{ }^{o p}\left(G_{i+1}, R\right)$ by a rule other than EC-Commit.

Finally Simple $\mathcal{T}$ when both FrontReapFree $\mathcal{T}$ and FrontCommitFree $\mathcal{T}$.

Lemma 14. Suppose high; $\Gamma \vdash^{\text {tso }} t$ and active $\mathcal{E}$ then $\mathcal{T}::(G, \mathcal{E}[W \mid t] \| \mathcal{o}) \Longrightarrow^{\text {tso* }}\left(G^{\prime}, \mathcal{E}\left[W \mid\left\langle L^{\prime}, \text { skip }\right\rangle_{\iota}\right] \| \mathcal{o}\right)$ for some $G^{\prime}, L^{\prime}$ and Simple $\mathcal{T}$.

Proof. Let $\langle L, c\rangle=t$ and proceed by an easy strong induction on size $c$. Because $c$ is high-typed it does not contain any occurrences of while, so if it takes a step other than EC-REAP, EC-COMMIT, or EC-FORK the size of $c$ decreases and we can conclude by the induction hypothesis.

If $c=$ skip we're done. Otherwise, observe that typing ensures $c$ contains no occurrences of fork, fence, sync, or holding. Therefore $c$ is not stuck and can take an eval-step that is not EC-REAP or EC-FORK. Conclude noting that $c$ steps to a smaller command.

Lemma 15 (Contextual compatibility for eval steps). Suppose active $\mathcal{E}$ and c.locks $\cap \lambda \subseteq$ L.locks where $\mathcal{E}=$ $(\lambda, \mathcal{C})$. Also assume that for all $\ell \in \lambda$ it is the case that wellStruct holding $\ell$ do $c$. If $\left(G, \mathcal{E}_{\emptyset}[W \mid\langle L, c\rangle]\right) \longrightarrow^{\text {eval }}$ $\left(G^{\prime}, \mathcal{E}_{\emptyset}\left[W \mid t^{\prime}\right] \| P^{\prime}\right)$ then $(G, \mathcal{E}[W \mid\langle L, c\rangle]) \longrightarrow^{\text {eval }}\left(G^{\prime}, \mathcal{E}\left[W \mid t^{\prime}\right] \| P^{\prime}\right)$ by a step rule other than EC-REAP.

Proof. by induction.

Lemma 16 (Contextual compatibility for arbitrary steps). Suppose active $\mathcal{E}$ and c.locks $\cap \lambda \subseteq$ L.locks where $\mathcal{E}=(\lambda, \mathcal{C})$. Also assume that for all $\ell \in \lambda$ it is the case that wellStruct holding $\ell$ do $c$. If $\left(G,\langle L, c\rangle_{\iota}\right) \longrightarrow^{o p}$ $\left(G^{\prime},\left\langle L^{\prime}, c^{\prime}\right\rangle_{\iota} \| P^{\prime}\right)$, then it is the case that $\left(G, \mathcal{E}\left[n i l \mid\langle L, c\rangle_{\iota}\right]\right) \longrightarrow^{o p}\left(G^{\prime}, \mathcal{E}\left[n i l \mid\left\langle L^{\prime}, c^{\prime}\right\rangle_{\iota}\right] \| P^{\prime}\right)$ by a step rule other than EC-REAP.

Proof. Commit-steps are trivial; use Lemma 15 for eval-steps.

Lemma 17 (Contextual compatibility for evaluation). Suppose $\mathcal{T}::\left(G,\langle L, c\rangle_{\iota} \| P\right) \Longrightarrow{ }^{\text {tso* }}\left(G^{\prime},\left\langle L^{\prime}, c^{\prime}\right\rangle_{\iota} \| P^{\prime}\right)$ and FrontReapFree $\mathcal{T}$. Assume for $\mathcal{E}=(\lambda, \mathcal{C})$ and both active $\mathcal{E}$ and c.locks $\cap \lambda \subseteq$ L.locks. Also assume that for all $\ell \in \lambda$ it is the case that wellStruct holding $\ell \mathbf{d o} c$. Then it is the case that $\mathcal{T}^{\prime}::$ $\left(G, \mathcal{E}\left[n i l \mid\langle L, c\rangle_{\iota}\right] \| P\right) \Longrightarrow{ }^{\text {tso* }}\left(G^{\prime}, \mathcal{E}\left[n i l \mid\left\langle L^{\prime}, c^{\prime}\right\rangle_{\iota}\right] \| P^{\prime}\right)$ where FrontReapFree $\mathcal{T}^{\prime}$

Proof. By an easy induction on the size of $\mathcal{T}$, using Lemma 16.

\subsection{Equivalences}

We define several forms of low equivalence. The various $\sim$ relations are used with all three systems - tso, sc, and $\mathrm{wb}$-introduced in this document, while the $\sim^{\text {tso }}$ relations are specialized for the Smith-Volpano-style system.

Definition $9\left(\sim_{\Gamma}\right)$.

1. $M_{1} \sim_{\Gamma} M_{2}$ iff for all $x$ such that $\Gamma(x)=$ low it is the case that $M_{1}(x)=M_{2}(x)$.

2. $W_{1} \sim_{\Gamma} W_{2}$ is defined as the least fixed point of the following implications. 
(a) nil $\sim_{\Gamma}$ nil

(b) $(X:=i):: W_{1} \sim_{\Gamma}(X:=i):: W_{2}$ when $W_{1} \sim_{\Gamma} W_{2}$

(c) $(X:=i):: W_{1} \sim_{\Gamma} W_{2}$ when $W_{1} \sim_{\Gamma} W_{2}$ and $\Gamma(X)=$ high

(d) $W_{1} \sim_{\Gamma}(X:=i):: W_{2}$ when $W_{1} \sim_{\Gamma} W_{2}$ and $\Gamma(X)=$ high

3. $S_{1} \sim_{\Gamma} S_{2}$ iff for all $X$ such that $\Gamma(X)=$ low it is the case that $S_{1}(X)=S_{2}(X)$.

Definition $10\left(\sim \sim_{\Gamma}^{\text {tso }}\right)$.

1. $L_{1} \sim_{\Gamma}^{\text {tso }} L_{2}$ iff each of the following holds:

(a) $L_{1} . w b \sim_{\Gamma} L_{2} . w b$

(b) $L_{1}$. mem $\sim_{\Gamma} L_{2}$. mem

(c) $L_{1}$. locks $=L_{2}$. locks

2. $t_{1} \sim_{\Gamma}^{\text {tso }} t_{2}$ is defined by the following introduction rules

(a) $\left\langle L_{1}, c\right\rangle_{\iota_{1}} \sim_{\Gamma}^{\text {tso }}\left\langle L_{2}, c\right\rangle_{\iota_{2}}$ when $L_{1} \sim_{\Gamma}^{\text {tso }} L_{2}$

(b) $\mathcal{E}\left[W_{1} \mid\left\langle L_{1}, c_{1}\right\rangle_{\iota_{1}}\right] \sim_{\Gamma}^{\text {tso }} \mathcal{E}\left[W_{2} \mid\left\langle L_{2}, c_{2}\right\rangle_{\iota_{2}}\right]$ when $L_{1} \sim_{\Gamma}^{\text {tso }} L_{2}$ and $W_{1} \sim_{\Gamma} W_{2}$ and both high; $\Gamma \vdash^{\text {tso }}$ $\left\langle L_{1}, c_{1}\right\rangle_{\iota_{1}}$ and high; $\Gamma \vdash^{\text {tso }}\left\langle L_{2}, c_{2}\right\rangle_{\iota_{2}}$.

3. $G_{1} \sim_{\Gamma}^{\text {tso }} G_{2}$ iff $G_{1}$. mem $\sim_{\Gamma} G_{2}$. mem and $G_{1}$.locks $=G_{2}$.locks.

4. $P_{1} \sim_{\Gamma}^{\text {tso }} P_{2}$ is defined by the least fixed point of the following implications.

(a) $\mathcal{O} \sim_{\Gamma}^{\text {tso }} \mathcal{o}$, always

(b) $t \| P_{1} \sim_{\Gamma}^{\text {tso }} P_{2}$ when high; $\Gamma \vdash^{\text {tso }} t$ and $P_{1} \sim_{\Gamma}^{\text {tso }} P_{2}$

(c) $P_{1} \sim_{\Gamma}^{\text {tso }} t \| P_{2}$ when high; $\Gamma \vdash^{\text {tso }} t$ and $P_{1} \sim_{\Gamma}^{\text {tso }} P_{2}$

(d) $t_{1}\left\|P_{1} \sim_{\Gamma}^{\text {tso }} t_{2}\right\| P_{2}$ when $t_{1} \sim_{\Gamma}^{\text {tso }} t_{2}$ and $P_{1} \sim_{\Gamma}^{\text {tso }} P_{2}$

5. $\left(G_{1}, P_{1}\right) \sim \sim_{\Gamma}^{\text {tso }}\left(G_{2}, P_{2}\right)$ when $G_{1} \sim_{\Gamma}^{\text {tso }} G_{2}$ and $P_{1} \underset{\Gamma}{\sim} \sim_{\Gamma}^{\text {tso }} P_{2}$.

Lemma 18. Each $\sim_{\Gamma}$ and $\sim^{\text {tso }}$ relation is an equivalence relation.

Proof. By inspection.

Lemma 19. If $P_{11}\left\|t_{1}\right\| P_{12} \sim_{\Gamma} P_{2}$ then $P_{2}=P_{21}\left\|P_{2}^{*}\right\| P_{22}$ where the following hold:

$$
\begin{aligned}
P_{21} & \sim_{\Gamma} P_{11} \\
P_{2}^{*} & \sim_{\Gamma} t_{1} \\
P_{22} & \sim_{\Gamma} P_{12} \\
P_{2}^{*} & \in\left\{\mathfrak{o}, t_{2} \| \text { o }\right\} \text { for some } t_{2}
\end{aligned}
$$

Lemma 20. Suppose $G_{1} \underset{\Gamma}{\sim \text { tso }} G_{2}$. Then $G_{1} \cup\{\ell\} \underset{\Gamma}{\sim_{\Gamma}^{\text {tso }}} G_{2} \cup\{\ell\}$.

Lemma 21. Suppose $L_{1} \sim_{\Gamma}^{\text {tso }} L_{2}$. Then $L_{1} \cup \lambda \sim_{\Gamma}^{\text {tso }} L_{2} \cup \lambda$.

Lemma 22. Suppose $L_{1} \sim_{\Gamma}^{\text {tso }} L_{2}$. Then $L_{1}+(X:=i) \sim_{\Gamma}^{\text {tso }} L_{2}+(X:=i)$.

Lemma 23. Suppose $L_{1} \sim_{\Gamma}^{\text {tso }} L_{2}$ and $\Gamma(X)=$ high. Then $L_{1}+(X:=i) \sim_{\Gamma}^{\text {tso }} L_{2}+(X:=j)$.

Lemma 24. Suppose $L_{1} \sim_{\Gamma}^{\text {tso }} L_{2}$. Then $L_{1}[x \mapsto i] \sim_{\Gamma}^{\text {tso }} L_{2}[x \mapsto i]$.

Lemma 25. Suppose $L_{1} \sim_{\Gamma}^{\text {tso }} L_{2}$ and $\Gamma(x)=$ high. Then $L_{1}\left[x \mapsto i_{1}\right] \sim_{\Gamma}^{\text {tso }} L_{2}\left[x \mapsto i_{2}\right]$.

Lemma 26. Suppose $G_{1}$. mem $\sim_{\Gamma} G_{2}$.mem and $L_{1} . w b \sim_{\Gamma} L_{2}$.wb and $\Gamma(X)=$ low. If $\left(G_{1} . m e m ; L_{1} . w b\right)[X] \Downarrow$ $i_{1}$ and $\left(G_{2}\right.$. mem; $\left.L_{2} . w b\right)[X] \Downarrow i_{2}$ then $i_{1}=i_{2}$.

Lemma 27. $G_{1} \sim \sim_{\Gamma}^{\text {tso }} G_{2}$ implies $G_{1}[X \mapsto i] \sim_{\Gamma}^{\text {tso }} G_{2}[X \mapsto i]$

Lemma 28. If $t_{1}\left\|\mathcal{o} \sim_{\Gamma}^{\text {tso }} t_{2}\right\| \mathcal{o}$ and $W_{1} \sim_{\Gamma} W_{2}$ then $\mathcal{E}\left[W_{1} \mid t_{1}\right]\left\|\mathcal{o} \sim_{\Gamma}^{\text {tso }} \mathcal{E}\left[W_{2} \mid t_{2}\right]\right\| \mathcal{o}$.

Lemma 29. $\left\langle L_{1}, c_{1}\right\rangle \sim_{\Gamma}^{\text {tso }}\left\langle L_{2}, c_{2}\right\rangle$ implies $L_{1} . w b \sim_{\Gamma}^{\text {tso }} L_{2}$.wb. 
Proof. Suppose we have $L_{1} \sim_{\Gamma}^{\text {tso }} L_{2}$, then we're done by definition. Otherwise $L_{1}$ and $L_{2}$ have write buffers with equivalent prefixes and suffixes. (The suffixes are both high-typed). These are also equivalent.

Lemma 30. Suppose $\left\langle L_{1}, c_{1}\right\rangle_{\iota_{1}} \sim_{\Gamma}^{\text {tso }}\left\langle L_{2}, c_{2}\right\rangle_{\iota_{2}}$ and $L_{1}^{*} \sim_{\Gamma}^{\text {tso }} L_{1}$. Then $\left\langle L_{1}^{*}, c_{1}\right\rangle_{\iota_{1}} \sim_{\Gamma}^{\text {tso }}\left\langle L_{2}, c_{2}\right\rangle_{\iota_{2}}$.

Proof. We proceed by considering two cases. Suppose that $c_{1}=c_{2}$ and $L_{1} \sim_{\Gamma}^{\text {tso }} L_{2}$; then we conclude using Lemma 18.

Suppose instead that

$$
\begin{aligned}
\left\langle L_{1}, c_{1}\right\rangle_{\iota_{1}} & =\mathcal{E}\left[W_{1} \mid\left\langle L_{10}, c_{10}\right\rangle_{\iota_{1}}\right] \\
\left\langle L_{2}, c_{2}\right\rangle_{\iota_{2}} & =\mathcal{E}\left[W_{2} \mid\left\langle L_{20}, c_{20}\right\rangle_{\iota_{2}}\right]
\end{aligned}
$$

with high; $\Gamma \vdash^{\text {tso }}\left\langle L_{10}, c_{10}\right\rangle_{\iota_{1}}$ and high; $\Gamma \vdash^{\text {tso }}\left\langle L_{20}, c_{20}\right\rangle_{\iota_{2}}$ and both $L_{10} \sim_{\Gamma}^{\text {tso }} L_{20}$ and $W_{1} \sim_{\Gamma} W_{2}$. Let $(\lambda, \mathcal{C})=\mathcal{E}$ and $W_{1}^{*}=L_{1}^{*}$. wb and define $L_{10}^{*}=\left(L_{1}^{*}\right.$.mem, $L_{1}^{*}$.locks $\left.\backslash \lambda, n i l\right)$. We want to find

$$
\left\langle L_{1}^{*}, c_{1}\right\rangle_{\iota_{1}}=\mathcal{E}\left[W_{1}^{*} \mid\left\langle L_{10}^{*}, c_{10}\right\rangle_{\iota_{1}}\right] \sim_{\Gamma}^{\text {tso }} \mathcal{E}\left[W_{2} \mid\left\langle L_{20}, c_{20}\right\rangle_{\iota_{2}}\right],
$$

which follows from three interesting properties.

- To establish high; $\Gamma \vdash^{\text {tso }}\left\langle L_{10}^{*}, c_{10}\right\rangle_{\iota_{1}}$, it is necessary to show $L_{10}^{*} \cdot$ locks $=\emptyset$ :

$$
\begin{aligned}
L_{10}^{*} \text {. locks } & =L_{1}^{*} \backslash \lambda & & \\
& =L_{1} \backslash \lambda & & \text { by defn. of } \sim_{\Gamma}^{\text {tso }} \\
& =L_{10} \backslash \lambda & & \text { by defn of } \mathcal{E} \text {-substitution } \\
& =\emptyset \backslash \lambda & & \text { by typing }
\end{aligned}
$$

- To show $W_{1}^{*} \sim_{\Gamma} W_{2}$ we use Lemma 29 to find $W_{1}^{*}=L_{1}^{*} \cdot w b \sim_{\Gamma} L_{1} \cdot w b \sim_{\Gamma} L_{2} . w b=W_{2}+L_{20} . w b$. Because $L_{20}$ has high type, it follows that $W_{1}^{*} \sim_{\Gamma} W_{2}+L_{20} . w b \sim_{\Gamma} W_{2}$.

- And $L_{10}^{*} \sim_{\Gamma}^{\text {tso }} L_{20}$ follows from Lemma 18 and the definition of $\sim_{\Gamma}^{\text {tso }}$.

Lemma 31. If $\left\langle(X:=i)+L_{1}, c_{1}\right\rangle_{\iota_{1}} \sim_{\Gamma}^{\text {tso }}\left\langle(X:=i)+L_{2}, c_{2}\right\rangle_{\iota_{2}}$ then $\left\langle L_{1}, c_{1}\right\rangle_{\iota_{1}} \sim_{\Gamma}^{\text {tso }}\left\langle L_{2}, c_{2}\right\rangle_{\iota_{2}}$.

Lemma 32. Suppose $L_{1} \sim_{\Gamma}^{\text {tso }} L_{2}$ and both high; $\Gamma \vdash^{\text {tso }} c_{1}$ and high; $\Gamma \vdash^{\text {tso }} c_{2}$. Then $\left\langle L_{1}, c_{1}\right\rangle_{\iota_{1}} \sim_{\Gamma}^{\text {tso }}\left\langle L_{2}, c_{2}\right\rangle_{\iota_{2}}$

Proof. Let $L_{1}^{\prime}=\left(L_{1} . m e m, \emptyset, n i l\right)$ and $L_{2}^{\prime}=\left(L_{2} . m e m, \emptyset, n i l\right)$. From $L_{1} \sim_{\Gamma}^{\text {tso }} L_{2}$, it follows that $W_{1}=$ $L_{1} . w b \sim_{\Gamma} L_{2} . w b=W_{2}$ and there is some $\lambda=L_{1}$.locks $=L_{2}$.locks. Conclude by defining $\mathcal{E}=(\lambda,[\cdot])$ and observing $\left\langle L_{1}, c_{1}\right\rangle_{\iota_{1}}=\mathcal{E}\left[W_{1} \mid\left\langle L_{1}^{\prime}, c_{1}\right\rangle_{\iota_{1}}\right] \sim_{\Gamma}^{\text {tso }} \mathcal{E}\left[W_{2} \mid\left\langle L_{2}^{\prime}, c_{2}\right\rangle_{\iota_{2}}\right]=\left\langle L_{2}, c_{2}\right\rangle_{\iota_{2}}$.

\subsection{Possibilistic Noninterference}

Definition 11 (Possibilistic noninterference). We say that command $c$ is possibilistically noninterfering (or possibilistically secure) under memory model $\mathrm{mm}$ and policy $\Gamma$ if for all $S_{1}, S_{2}$ such that $S_{1} \sim_{\Gamma} S_{2}$, if $\left(\left(S_{1}\right.\right.$, Lock $\left.),\left\langle L_{\oslash}, c\right\rangle\right) \Longrightarrow^{\mathrm{mm} *}\left(G_{1}^{\prime}, \mathrm{o}\right)$ then there exists $G_{2}^{\prime}$ such that $\left(\left(S_{2}\right.\right.$, Lock $\left.),\left\langle L_{\oslash}, c\right\rangle\right) \Longrightarrow^{\mathrm{mm} *}\left(G_{2}^{\prime}, \mathrm{o}\right)$ and $G_{1}^{\prime}$.mem $\sim_{\Gamma} G_{2}^{\prime}$. mem.

\subsection{Security}

Lemma 33. If $\mathcal{E}=(\lambda, \mathcal{C})$ and $(G, \mathcal{E}[W \mid\langle L, c\rangle]) \longrightarrow^{\text {eval }}\left(G^{\prime}, P^{\prime}\right)$ and L.locks $\supseteq \lambda \cap$ c.locks, then either $c=$ skip or both $P^{\prime}=\mathcal{E}\left[W \mid\left\langle L^{\prime}, c^{\prime}\right\rangle\right] \| P_{0}^{\prime}$ and $\left(G, \mathcal{E}_{\emptyset}[W \mid\langle L, c\rangle]\right) \longrightarrow^{\text {eval }}\left(G^{\prime}, \mathcal{E}_{\emptyset}\left[W \mid\left\langle L^{\prime}, c^{\prime}\right\rangle\right] \| P_{0}^{\prime}\right)$.

Proof. by simple induction. The interesting cases occur when $c$ has form sync or holding; these cases work because we know that if $c$ references a lock in $\mathcal{E}$, that lock also occurs in $L$.

Lemma 34. Whenever high; $\Gamma \vdash^{\text {tso }} c$ it is the case that c.locks $=\emptyset$.

Proof. by induction.

Lemma 35 (Global confinement). Suppose high; $\Gamma \vdash^{\text {tso }} t$ and $(G, \mathcal{E}[W \mid t]) \longrightarrow^{o p}\left(G^{\prime}, P^{\prime}\right)$. If $P^{\prime}=\mathrm{o}$ or $P^{\prime}=\mathcal{E}\left[W \mid t^{\prime}\right] \| P_{0}^{\prime}$ then $(G, \mathcal{E}[W \mid t] \| \mathcal{o}) \sim \sim_{\Gamma}^{\text {tso }}\left(G^{\prime}, P^{\prime}\right)$. 
Proof. Assume $P^{\prime}=\mathrm{o}$ or $P^{\prime}=\mathcal{E}\left[W \mid t^{\prime}\right] \| P_{0}^{\prime}$. Observe that either op $\neq$ commit or $W=$ nil.

First we demonstrate $G \sim_{\Gamma}^{\text {tso }} G^{\prime}$. It's necessary to show $G$.locks $=G^{\prime}$. locks, which follows from inverting the typing relation finitely many times and observing that $\longrightarrow^{o p}$ cannot contain a (nested) use of ECSyncACquire or EC-HoldRelease. Consider an arbitrary global variable $X$; it remains to show that $G(X)=G^{\prime}(X)$ whenever $\Gamma(X)=$ low. Suppose that op $\neq$ commit; then G.mem $=G^{\prime}$.mem and we're done. Instead suppose that $o p=$ commit and, consequently, $W=$ nil. Then $G^{\prime}=G[Y \mapsto i]$ where $t . w b=(Y:=i):: W_{0}$. Inverting the typing of $t . w b$ yields $h i g h \sqsubseteq \Gamma(Y)$ so $X \neq Y$ and $G(X)=G^{\prime}(X)$.

Second we must show $\mathcal{E}[W \mid t] \| \mathcal{o} \sim_{\Gamma}^{\text {tso }} P^{\prime}$. If $P^{\prime}=\mathrm{o}$ then the process stepped by EC-REAP, so $\mathcal{E}=(\emptyset,[\cdot])$ and $\mathcal{E}[W \mid t]=t$. Thus we can conclude by noting high; $\Gamma \vdash^{\text {tso }} t$ implies $\mathcal{E}[W \mid t]\|\mathcal{o}=t\| \mathcal{o} \sim_{\Gamma}^{\text {tso }} \mathcal{o}=P^{\prime}$. If instead $P^{\prime}=\mathcal{E}\left[W \mid t^{\prime}\right] \| P_{0}^{\prime}$ then it suffices to show high; $\Gamma \vdash^{\text {tso }} t^{\prime}$ and $\overline{p c} ; \Gamma \vdash^{\text {tso }} P_{0}^{\prime}$ for high $\sqsubseteq \overline{p c}$, which follow from Lemma 10.

Lemma 36 (Local Confinement). Suppose high; $\Gamma \vdash^{\text {tso }} t$ and $(G, \mathcal{E}[W \mid t]) \longrightarrow{ }^{o p}\left(G^{\prime}, \mathcal{E}\left[W \mid t^{\prime}\right] \| P^{\prime}\right)$. Then $\mathcal{E}[W \mid t] \sim_{\Gamma}^{\text {tso }} \mathcal{E}\left[W \mid t^{\prime}\right]$

Proof. Let $t=\langle L, c\rangle$ and $t^{\prime}=\left\langle L^{\prime}, c^{\prime}\right\rangle$. As in the proof of Lemma 35, a low write, a low commit, a lock, or an unlock would contradict $c^{\prime}$ 's high type. Therefore $L \sim_{\Gamma}^{\text {tso }} L^{\prime}$. It remains remains to show that $h i g h ; \Gamma \vdash^{\text {tso }}\left\langle L^{\prime}, c^{\prime}\right\rangle$ and $\overline{p c} ; \Gamma \vdash^{\text {tso }} P^{\prime}$ for high $\sqsubseteq \overline{p c}$, which follow from Lemma 10.

Lemma 37 (Contextual confinement, fork free). Suppose $\mathcal{T}::(G, \mathcal{E}[W \mid t] \| \mathcal{o}) \Longrightarrow{ }^{\text {tso* }}\left(G^{\prime}, \mathcal{E}\left[W^{\prime} \mid s^{\prime}\right] \| \mathcal{o}\right)$ and Simple $\mathcal{T}$. Further suppose high; $\Gamma \vdash^{\text {tso }} t$. Then there is some $t^{\prime}$ such that following hold:

$$
\begin{aligned}
& \mathcal{E}\left[W \mid t^{\prime}\right]=\mathcal{E}\left[W^{\prime} \mid s^{\prime}\right] \\
& \mathcal{E}[W \mid t] \sim \Gamma \text { tso } \\
& G\left[W \mid t^{\prime}\right] \\
& G \sim \sim_{\Gamma}^{\text {tso }} \\
& G^{\prime}
\end{aligned}
$$

Proof. By induction on the length of $\mathcal{T}$. If $\mathcal{T}$ contains a single element then $G^{\prime}=G, \mathcal{E}[W \mid t]=\mathcal{E}\left[W^{\prime} \mid s^{\prime}\right]$ and we conclude using that $\sim_{\Gamma}^{\text {tso }}$ is an equivalence relation.

Suppose $\mathcal{T}$ contains $n>1$ elements. Because high commands cannot contain forks, $\mathcal{T}$ witnesses an evaluation sequence of the following form:

$$
(G, \mathcal{E}[W \mid t]) \Longrightarrow{ }^{\text {tso }}\left(G_{1}, \mathcal{E}\left[W_{1} \mid s_{1}\right]\right) \Longrightarrow^{\text {tso* }}\left(G^{\prime}, \mathcal{E}\left[W^{\prime} \mid s^{\prime}\right]\right)
$$

Because Simple $\mathcal{T}$ the first step is not a commit, so $W_{1}+s . w b=W+t . w b$ or-if the step is by a write$W_{1}+s_{1} \cdot w b=W+t . w b+(X:=i):: n i l$. In either case we can define $W_{t_{1}}$ such that $W_{1}+s_{1} \cdot w b=W+W_{t_{1}}$. Define $t_{1}=\left\langle\left(s_{1}\right.\right.$.mem, $s_{1}$.locks, $\left.\left.W_{t_{1}}\right), s_{1} . c m d\right\rangle$ and observe that $\mathcal{T}$ also witnesses the following:

$$
(G, \mathcal{E}[W \mid t]) \Longrightarrow^{\text {tso }}\left(G_{1}, \mathcal{E}\left[W \mid t_{1}\right]\right)=\left(G_{1}, \mathcal{E}\left[W_{1} \mid s_{1}\right]\right) \Longrightarrow^{\text {tso* }}\left(G^{\prime}, \mathcal{E}\left[W^{\prime} \mid s^{\prime}\right]\right)
$$

By the induction hypothesis $\left(G^{\prime}, \mathcal{E}\left[W^{\prime} \mid s^{\prime}\right]\right)=\left(G^{\prime}, \mathcal{E}\left[W \mid t^{\prime}\right]\right)$ for some $t^{\prime}$. Additionally $G_{1} \sim_{\Gamma}^{\text {tso }} G^{\prime}$ and $\mathcal{E}\left[W \mid t_{1}\right] \sim_{\Gamma}^{\text {tso }} \mathcal{E}\left[W \mid t^{\prime}\right]=\mathcal{E}\left[W^{\prime} \mid s^{\prime}\right]$. Using the transitivity of $\sim_{\Gamma}^{\text {tso }}$, it remains to show $G \sim_{\Gamma}^{\text {tso }} G_{1}$ and $\mathcal{E}[W \mid t] \sim_{\Gamma}^{\text {tso }} \mathcal{E}\left[W \mid t_{1}\right]$. These are consequences of Lemmas 35 and 36, respectively.

Lemma 38 (SV Expression Confinement). Suppose $L_{1} \sim_{\Gamma}^{\text {tso }} L_{2}$. Then low; $\Gamma \vdash^{\text {tso }}$ a implies $i_{1}=i_{2}$ when $L_{1}[a] \Downarrow i_{1}$ and $L_{2}[a] \Downarrow i_{2}$. Likewise, low; $\Gamma \vdash^{\text {tso }} b$ implies $\beta_{1}=\beta_{2}$ when $L_{1}[b] \Downarrow \beta_{1}$ and $L_{2}[b] \Downarrow \beta_{2}$.

Proof. by induction

Lemma 39 (SV Commit step security). Suppose $\left(G_{1},\left\langle L_{1}, c_{1}\right\rangle_{\iota_{1}}\right) \longrightarrow^{\text {commit }}\left(G_{1}^{\prime}, P_{1}^{\prime}\right)$ and pc; $\Gamma \vdash^{\text {tso }}\left\langle L_{1}, c_{1}\right\rangle_{\iota_{1}}$. Further assume both $\left\langle L_{1}, c_{1}\right\rangle_{\iota_{1}} \sim_{\Gamma}^{\text {tso }} t_{2}$ and $G_{1} \sim_{\Gamma}^{\text {tso }} G_{2}$. Then there exist $L_{1}^{\prime}$, $G_{2}^{\prime}$, and $t_{2}^{\prime}$ such that $P_{1}^{\prime}=$ $\left\langle L_{1}^{\prime}, c_{1}\right\rangle_{\iota_{1}} \| \mathcal{o}$ and $G_{1}^{\prime} \sim_{\Gamma}^{\text {tso }} G_{2}^{\prime}$ and $\left\langle L_{1}^{\prime}, c_{1}\right\rangle_{\iota_{1}} \sim_{\Gamma}^{\text {tso }} t_{2}^{\prime}$ and $\left(G_{2}, t_{2} \| \mathcal{o}\right) \Longrightarrow^{\text {tso* }}\left(G_{2}^{\prime}, t_{2}^{\prime} \| \mathcal{o}\right)$.

Proof. Let $\left\langle L_{2}, c_{2}\right\rangle_{\iota_{2}}=t_{2}$. Proof is by induction on the structure of $L_{2}$.wb. Inverting the evaluation relation shows for some $X, i$, and $L_{10}$, and that

$$
\begin{aligned}
L_{1} & =(X:=i)+L_{10} \\
G_{1}^{\prime} & =G_{1}[X \mapsto i] \\
P_{1}^{\prime} & =\left\langle L_{10}, c_{1}\right\rangle \| \mathcal{o} .
\end{aligned}
$$

Let $L_{10}$ be the witness to $L_{1}^{\prime}$. 
If $\Gamma(X)=$ high then by definition $G_{1}^{\prime} \sim_{\Gamma}^{\text {tso }} G_{1} \sim_{\Gamma}^{\text {tso }} G_{2}$ and $L_{1} \sim_{\Gamma}^{\text {tso }} L_{10}$. So taking $G_{2}^{\prime}=G_{2}$ and $t_{2}^{\prime}=t_{2}$, it suffices to show $\left\langle L_{10}, c_{1}\right\rangle_{\iota_{1}} \sim_{\Gamma}^{\text {tso }}\left\langle L_{2}, c_{2}\right\rangle_{\iota_{2}}$, a consequence of Lemma 30.

Suppose instead that $\Gamma(X)=$ low. Were $L_{2} . w b$ empty this would contradict the assumption that $t_{1} \sim_{\Gamma}^{\text {tso }}$ $t_{2}$, making the conclusion trivial. If $L_{2} . w b=(X:=j)+L_{20}$ then, by the definition of $\sim_{\Gamma}, i=j$ and $L_{10} \cdot w b \sim_{\Gamma} L_{20} \cdot w b$. Because $\left(G_{2}, t_{2}\right) \longrightarrow^{\text {commit }}\left(G_{2}[X \mapsto i],\left\langle L_{20}, c_{2}\right\rangle_{\iota_{2}}\right)$ it suffices to show $G_{1}[X \mapsto i] \sim_{\Gamma}^{\text {tso }}$ $G_{2}[X \mapsto i]$, which follows from by Lemma 27 , and $\left\langle L_{10}, c_{1}\right\rangle \sim_{\Gamma}^{\text {tso }}\left\langle L_{20}, c_{2}\right\rangle_{\iota_{2}}$, which follows from Lemma 31 .

Finally if $L_{2} . w b=(Y:=j)+L_{20}$ with $Y \neq X$ the definition of $\sim_{\Gamma}$ requires that $\Gamma(Y)=$ high. So $\left(G_{2}, t_{2}\right) \Longrightarrow{ }^{\text {tso }}\left(G_{2}[Y \mapsto j],\left\langle L_{20}, c_{2}\right\rangle\right)$ where $G_{1} \sim_{\Gamma}^{\text {tso }} G_{2}[Y \mapsto j]$ and $\left\langle L_{1}, c_{1}\right\rangle \sim_{\Gamma}^{\text {tso }}\left\langle L_{20}, c_{2}\right\rangle$. The induction hypothesis yields

$$
\left(G_{2}[Y \mapsto j],\left\langle L_{20}, c_{2}\right\rangle\right) \Longrightarrow^{\mathrm{tso} *}\left(G_{2}^{\prime}, t_{2}^{\prime} \| \mathrm{o}\right)
$$

where $G_{1}^{\prime} \sim_{\Gamma}^{\text {tso }} G_{2}^{\prime}$ and $\left\langle L_{10}, c_{1}\right\rangle_{\iota_{1}} \sim_{\Gamma}^{\text {tso }} t_{2}^{\prime}$. Conclude by deriving that $\left(G_{2}, t_{2}\right) \Longrightarrow^{\text {tso* }}\left(G_{2}^{\prime}, t_{2}^{\prime} \| \mathrm{o}\right)$.

Lemma 40 (SV Eval step security). Suppose $\left(G_{1}, t_{1}\right) \longrightarrow^{\text {eval }}\left(G_{1}^{\prime}, P_{1}^{\prime}\right)$ and $p c ; \Gamma \vdash^{\text {tso }} t_{1}$ and wellStruct $t_{1}$. Further assume both $t_{1} \sim_{\Gamma}^{\text {tso }} t_{2}$ and $G_{1} \sim_{\Gamma}^{\text {tso }} G_{2}$. Then there exist $G_{2}^{\prime}$ and $P_{2}^{\prime}$ such that $\left(G_{2}, t_{2}\right) \Longrightarrow{ }^{\text {tso* }}\left(G_{2}^{\prime}, P_{2}^{\prime}\right)$ and $\left(G_{1}^{\prime}, P_{1}^{\prime}\right) \sim_{\Gamma}^{\text {tso }}\left(G_{2}^{\prime}, P_{2}^{\prime}\right)$.

Proof. Let $t_{1}=\left\langle L_{1}, c_{1}\right\rangle_{\iota}$. We strengthen the induction hypothesis by also requiring $P_{2}^{\prime}$ satisfy the following property: When $P_{1}^{\prime}=t_{10}^{\prime} \| P_{10}^{\prime}$ then $P_{2}^{\prime}=t_{20}^{\prime} \| P_{20}^{\prime}$ and FrontReapFree $\mathcal{T}$ where $\mathcal{T}::\left(G_{2}, t_{2}\right) \Longrightarrow^{\text {tso* }}\left(G_{2}^{\prime}, P_{2}^{\prime}\right)$, and both $t_{10}^{\prime} \sim_{\Gamma}^{\text {tso }} t_{20}^{\prime}$ and $P_{10}^{\prime} \sim_{\Gamma}^{\text {tso }} P_{20}^{\prime}$. Proceed by induction on the structure of $c_{1}$, grouping cases in a non-standard way.

To avoid pointless textual copying we will let several cases refer to other parts of the argument. There is no circularity and the dependencies are as follows. In Case 2, subcases EC-HoldSTEP and EC-SEQStruCT refer to Case 1 and no other (sub)cases. Case 1 refers to Case 2, subcases EC-HoldRelease, EC-Reap, EC-SEQSKIP, and no other (sub)cases.

Consider the two principle cases arising from $t_{1} \underset{\Gamma}{\sim \text { tso }} t_{2}$.

- Case 1: $t_{1}=\mathcal{E}\left[W_{1} \mid\left\langle L_{10}, c_{10}\right\rangle\right]$ and $t_{2}=\mathcal{E}\left[W_{2} \mid\left\langle L_{20}, c_{20}\right\rangle\right]$ where high; $\Gamma \vdash^{\text {tso }}\left\langle L_{10}, c_{10}\right\rangle$ and $h i g h ; \Gamma \vdash^{\text {tso }}$ $\left\langle L_{20}, c_{20}\right\rangle$ and both $L_{10} \sim_{\Gamma}^{\text {tso }} L_{20}$ and $W_{1} \sim_{\Gamma} W_{2}$. Lemmas 33 and 34 show we must examine two situations:

- Suppose $c_{10}=$ skip. We will show that $t_{2}$ evaluates to a state such that we can finish by copying reasoning from the EC-HoldSteP, EC-SEQSKIP, or EC-REAP cases below. Lemma 14 gives a simple derivation of

$$
\left(G_{2}, \mathcal{E}_{2}\left[W_{1} \mid\left\langle L_{20}, c_{20}\right\rangle\right]\right) \Longrightarrow{ }^{\text {tso* }}\left(G_{20}^{\prime}, \mathcal{E}\left[W_{2} \mid\left\langle L_{20}^{\prime}, \text { skip }\right\rangle\right] \| \text { o }\right) .
$$

(Note that active $\mathcal{E}$ because $t_{1}$ steps and $L_{10}$.locks $=\emptyset$.) It now suffices to find witnesses $G_{2}^{\prime}$ and $P_{2}^{\prime}$ such that $\left(G_{20}^{\prime}, \mathcal{E}\left[W_{2} \mid\left\langle L_{20}^{\prime}\right.\right.\right.$, skip $\left.\rangle\right] \|$ o $) \Longrightarrow{ }^{\text {tso* }}\left(G_{2}^{\prime}, P_{2}^{\prime}\right)$ and $G_{1}^{\prime} \sim_{\Gamma}^{\text {tso }} G_{2}^{\prime}$ and $P_{2}^{\prime}$ satisfies the appropriate properties (above). Copying from case 2 can provide such witnesses, but requires we first demonstrate two equivalences. $G_{1} \sim_{\Gamma}^{\text {tso }} G_{2} \sim_{\Gamma}^{\text {tso }} G_{20}^{\prime}$ and $t_{1} \sim_{\Gamma}^{\text {tso }} \mathcal{E}\left[W_{2} \mid\left\langle L_{20}, c_{20}\right\rangle\right] \sim_{\Gamma}^{\text {tso }}$ $\mathcal{E}\left[W_{2} \mid\left\langle L_{20}^{\prime}, c_{20}^{\prime}\right\rangle\right]$ follow from Lemma 37 and the transitivity of $\sim_{\Gamma}^{\text {tso }}$.

- Suppose instead that, for some $t_{10}^{\prime}$, it is the case that $P_{1}^{\prime}=\mathcal{E}\left[W \mid t_{10}^{\prime}\right] \| P_{10}^{\prime}$ and $\left(G_{1}, \mathcal{E}_{\emptyset}\left[W_{1} \mid\left\langle L_{10}, c_{10}\right\rangle\right]\right) \longrightarrow{ }^{\text {eval }}$ $\left(G_{1}^{\prime}, \mathcal{E}_{\emptyset}\left[W_{1} \mid t_{10}^{\prime}\right] \| P_{10}^{\prime}\right)$. Take witnesses $G_{2}^{\prime}$ and $P_{2}^{\prime}$ to be $G_{2}$ and $P_{2}$. It suffices to show that $G_{1} \sim \sim_{\Gamma}^{\text {tso }} G_{1}^{\prime}$ and $t_{1} \| \mathcal{o} \sim \sim_{\Gamma}^{\text {tso }} P_{1}^{\prime}$. The former is a consequence of Lemma 35 . Toward the latter we use Lemma 12 to establish that $\overline{p c} ; \Gamma \vdash^{\text {tso }} P_{1}^{\prime}$ where high $\sqsubseteq \overline{p c}$ so $P_{10}^{\prime} \sim_{\Gamma}^{\text {tso }}$ o. It remains to show that $t_{1} \sim_{\Gamma}^{\text {tso }} \mathcal{E}\left[W \mid t_{10}^{\prime}\right]$, which follows from Lemmas $1,6,8,15,34$, and 36 . .

- Case 2: $t_{2}=\left\langle c_{2}, L_{2}\right\rangle$ where $c_{1}=c_{2}$ and $L_{1} \sim_{\Gamma}^{\text {tso }} L_{2}$. Continue by inverting the $\longrightarrow{ }^{\text {eval }}$ relation.

EC-Store: Here $c_{1}=X:=x$. If $\Gamma(x)=$ low, the definition of $\sim_{\Gamma}^{\text {tso }}$ shows $L_{1}(x)=L_{2}(x)$ and we conclude using Lemma 22. Otherwise $\Gamma(x)=h i g h$, inverting typing rule TSO-STORE gives $\Gamma(X)=h i g h$, and the result follows from Lemma 23 .

EC-LOAD: Here $c_{1}=x:=Y$. If $\Gamma(Y)=$ low then, by Lemma 26, $\left(G_{1} . m e m ; L_{1} . w b\right)[Y] \Downarrow i$ and $\left(G_{2} . m e m ; L_{2} . w b\right)[Y] \Downarrow i$ for some $i$. Conclude using Lemma 24. Suppose instead $\Gamma(Y)=h i g h$. Inverting typing rule TSO-LOAD shows $\Gamma(x)=h i g h$ and we conclude via Lemma 25 .

EC-EvalExp: Here $c_{1}=x:=a$. Suppose low $\vdash \Gamma: a$ then by Lemma 38 there exists a unique $i$ such that $L_{1}[a] \Downarrow i$ and $L_{2}[a] \Downarrow i$, and we can conclude via Lemma 24. Suppose instead high $\vdash \Gamma: a$, then by inverting typing TSO-EvalExP we find $\Gamma(x)=$ high. Conclude via Lemma 25. 
EC-SYNCACQUIRE: Here $c_{1}=\operatorname{sync} \ell$ do $c$ with $\ell \in G_{1}$ and $L_{1} . w b=n i l$. As $L_{1} \sim_{\Gamma}^{\text {tso }} L_{2}$, any store $(X:=i)$ in $L_{2} . w b$ must have $\Gamma(X)=h i g h$. As in the $o p=$ commit case use an inner induction on the structure of $L_{2}$.wb to find $G_{20}$ and $L_{20}$ where $\left(G_{2}, t_{2} \| \mathcal{o}\right) \Longrightarrow{ }^{\text {tso* }}\left(G_{20},\left\langle L_{20}\right.\right.$, sync $\ell$ do $\left.\left.c\right\rangle \| \mathcal{o}\right)$ and both $G_{20} \sim_{\Gamma}^{\text {tso }} G_{2} \sim_{\Gamma}^{\text {tso }} G_{1}$ and $L_{20} \sim_{\Gamma}^{\text {tso }} L_{2} \sim_{\Gamma}^{\text {tso }} L_{1}$. Via the first equivalence, $\ell \in G_{20}$ so using EC-SYNCACQUIRE we find $\left(G_{2}, t_{2} \| \mathrm{o}\right) \Longrightarrow^{\text {tso* }}\left(G_{20},\left\langle L_{20}\right.\right.$, holding $\ell$ do $\left.\left.c\right\rangle \| \mathcal{o}\right)$.

EC-HoldRelease, ec-Fence, ec-Fork: Similar to the EC-SyncAcquire case.

EC-SyncReEnter: Trivial after noting $L_{1} \sim_{\Gamma}^{\text {tso }} L_{2}$ gives $L_{1}$.locks $=L_{2}$.locks.

EC-HoldSteP: Here, $c_{1}=$ holding $\ell$ do $c$ and $P_{1}^{\prime}=\left\langle L_{1}^{\prime} \text {, holding } \ell \text { do } c_{10}^{\prime}\right\rangle_{\iota} \| P_{10}^{\prime}$.

Inversion and the induction hypothesis give

$$
\begin{gathered}
\ell \in L_{1} \\
\left(G_{1},\left\langle L_{1}, c\right\rangle_{\iota}\right) \longrightarrow^{\text {eval }}\left(G_{1}^{\prime},\left\langle L_{1}^{\prime}, c_{10}^{\prime}\right\rangle_{\iota} \| P_{10}^{\prime}\right) \\
\mathcal{T}::\left(G_{2},\left\langle L_{2}, c\right\rangle_{\iota}\right) \Longrightarrow^{\text {tso* }}\left(G_{2}^{\prime},\left\langle L_{2}^{\prime}, c_{20}^{\prime}\right\rangle_{\iota} \| P_{20}^{\prime}\right) .
\end{gathered}
$$

FrontReapFree $\mathcal{T}$

From $L_{1} \sim_{\Gamma}^{\text {tso }} L_{2}$ it follows that $\ell \in L_{2}$ and we can define $\mathcal{E}=(\{\ell\}$, holding $\ell$ do [.] $)$ where active $\mathcal{E}$ and $t_{2}=\mathcal{E}\left[n i l \mid\left\langle L_{2}, c\right\rangle_{\iota}\right]$. By Lemma 17,

$$
\begin{array}{cl}
\mathcal{T}^{\prime}::\left(G_{2}, t_{2} \| \mathcal{O}\right) & = \\
\Longrightarrow & \left(G_{2}, \mathcal{E}\left[n i l \mid\left\langle L_{2}, c\right\rangle_{\iota}\right]\right) \\
& \left(G_{2}^{\prime}, \mathcal{E}\left[n i l \mid\left\langle L_{2}^{\prime}, c_{20}^{\prime}\right\rangle_{\iota}\right] \| P_{20}^{\prime}\right)
\end{array}
$$

where FrontReapFree $\mathcal{T}^{\prime}$.

It is necessary to show the following:

$$
\begin{aligned}
& G_{1}^{\prime} \sim_{\Gamma}^{\text {tso }} G_{2}^{\prime}
\end{aligned}
$$

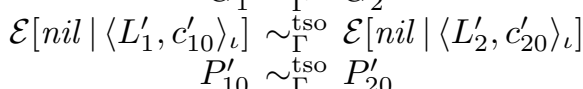

These are implied by the above use of induction hypothesis and Lemma 28.

eC-SeqStruct: Similar to eC-HoldStep.

EC-SEQSKIP: Immediate.

EC-IFTRuE: Here $c_{1}=c_{2}=$ if $b$ do $c_{t}$ else $c_{f}$ and inversion shows that $L_{1}[b] \Downarrow$ true and $P_{1}^{\prime}=\left\langle L_{1}, c_{t}\right\rangle$.

Suppose that it's not the case that $\Gamma \vdash b$ : low. Then inverting the typing relation shows both $h i g h ; \Gamma \vdash^{\text {tso }} c_{t}$ and $h i g h ; \Gamma \vdash^{\text {tso }} c_{f}$. Thread $t_{2}$ could potentially step to configuration $\left(G_{2},\left\langle L_{2}, c_{t}\right\rangle \| \mathcal{o}\right)$ or to configuration $\left(G_{2},\left\langle L_{2}, c_{f}\right\rangle \| \mathcal{o}\right)$. Without loss of generality assume the latter. Use Lemma 32 to establish $\left\langle L_{1}, c_{t}\right\rangle \sim_{\Gamma}^{\text {tso }}\left\langle L_{2}, c_{f}\right\rangle$. All other goals are immediate.

Suppose instead that $\Gamma \vdash b$ : low. Then Lemma 38 shows $L_{2}[b] \Downarrow$ true and we have a reap-free trace showing $\left(G_{2}, t_{2}\right) \Longrightarrow^{\text {tso* }}\left(G_{2},\left\langle L_{2}, c_{t}\right\rangle \| \mathcal{o}\right)$.

ec-IfFalse, ec-WhileTrue, ec-WhileFalse: Similar to, or simpler than, the EC-IfTrue case.

EC-REAP: Immediate, noting that the trace we construct need not be FrontReapFree.

Theorem 1 (SV Security). Suppose $\left(G_{1}, P_{1}\right) \sim_{\Gamma}^{\text {tso }}\left(G_{2}, P_{2}\right)$ and $\overline{p c} ; \Gamma \vdash^{\text {tso }} P_{1}$ and wellStruct $P_{1}$. Suppose also that $\left(G_{1}, P_{1}\right) \Longrightarrow{ }^{\text {tso }}\left(G_{1}^{\prime}, P_{1}^{\prime}\right)$. Then there exists $G_{2}^{\prime}, P_{2}^{\prime}$ such that $\left(G_{1}^{\prime}, P_{1}^{\prime}\right) \sim_{\Gamma}^{\text {tso }}\left(G_{2}^{\prime}, P_{2}^{\prime}\right)$ and $\left(G_{2}, P_{2}\right) \Longrightarrow^{\text {tso* }}\left(G_{2}^{\prime}, P_{2}^{\prime}\right)$.

Proof. Inverting the tso-evaluation relation and appealing to Lemma 19 gives

$$
\begin{aligned}
& P_{1}=P_{11}\left\|t_{1}\right\| P_{12} \\
& P_{2}=P_{21}\left\|P_{2}^{*}\right\| P_{22} \\
& P_{1}^{\prime}=P_{11}\left\|Q_{1}\right\| P_{12}
\end{aligned}
$$

where $P_{2}^{*}$ contains at most one thread (i.e., $P_{2}^{*} \in\left\{\mathfrak{o}, t_{2} \| \mathfrak{o}\right\}$ for some $t_{2}$ ) and the following hold:

$$
\left(G, t_{1}\right) \longrightarrow^{o p}\left(G_{1}^{\prime}, Q_{1}\right)
$$




$$
\begin{aligned}
& P_{11} \sim_{\Gamma}^{\text {tso }} P_{21} \\
& t \| \mathcal{o} \sim_{\Gamma}^{\text {tso }} P_{2}^{*} \\
& P_{12} \sim_{\Gamma}^{\text {tso }} P_{22}
\end{aligned}
$$

It suffices to show that there exists $G_{2}^{\prime}$ and $Q_{2}$ such that $\left(G_{1}^{\prime}, Q_{1}\right) \sim_{\Gamma}^{\text {tso }}\left(G_{2}^{\prime}, Q_{2}\right)$ and $\left(G_{2}, P_{2}^{*}\right) \Longrightarrow^{\text {tso* }}\left(G_{2}^{\prime}, Q_{2}\right)$. (Observe that while we could rename threads in $Q_{2}$, we do not need to; thread names are only really relevant for the data-race freedom argument.) Inspecting the definition of $\sim_{\Gamma}^{\text {tso }}$ shows there are only three ways in which to find $t \| \mathcal{O} \sim_{\Gamma}^{\text {tso }} P_{2}^{*}$. Proceed by case analysis.

First suppose that that the equivalence arises from Definition 10, clause 4b. Here high; $\Gamma \vdash^{\text {tso }} t_{1}$ and via Lemma $35,\left(G_{1}, t \|\right.$ o $) \sim_{\Gamma}^{\text {tso }}\left(G_{1}^{\prime}, Q_{1}\right)$. Note that to apply Lemma 35 we take $\mathcal{E}=(\emptyset,[\cdot])$ and $W=$ nil. Conclude using Lemma 18 and taking $G_{2}$ and $P_{2}^{*}$ as existential witnesses $G_{2}^{\prime}$ and $Q_{2}$.

Second suppose that that the equivalence arises from Definition 10, clause 4c. Here $P_{2}^{*}=t_{2} \| \mathcal{o}$ where high; $\Gamma \vdash^{\text {tso }} t_{2}$ and $t_{1} \sim_{\Gamma}^{\text {tso }} \mathcal{o}$. From $t_{1} \sim_{\Gamma}^{\text {tso }} \mathcal{o}$ it follows that high; $\Gamma \vdash^{\text {tso }} t_{1}$. Again taking $G_{2}$ and $P_{2}^{*}$ to be witnesses $G_{2}^{\prime}$ and $Q^{\prime}$ conclude with the following equational reasoning:

$$
\begin{array}{ccll}
\left(G^{\prime}, Q_{1}\right) & \sim_{\Gamma}^{\text {tso }} & \left(G_{1}, t_{1} \| \mathrm{o}\right) & \text { by Lemma } 35 \\
& \sim_{\Gamma}^{\text {tso }} & \left(G_{1}, \mathcal{o}\right) & \\
& \sim_{\Gamma}^{\text {tso }} & \left(G_{2}, \mathcal{o}\right) & \text { by assumption } \\
& \sim_{\Gamma}^{\text {tso }} & \left(G_{2}, t_{2} \| \mathrm{o}\right) & \\
& = & \left(G_{2}, P_{2}^{*}\right) &
\end{array}
$$

Third suppose that that the equivalence arises from Definition 10, clause $4 \mathrm{~d}$. Here $P_{2}^{*}=t_{2} \|$ o for some $t_{2}$ with $t_{1} \sim_{\Gamma}^{\text {tso }} t_{2}$. Finitely many inversions of the typing relation show $p c ; \Gamma \vdash^{\text {tso }} t_{1}$ for some $p c$. Similarly, wellStruct $t_{1}$. Conclude via Lemmas 39 and 40.

Corollary 1. Suppose $\left(G_{1}, P_{1}\right) \underset{\Gamma}{\sim} \sim_{\Gamma}^{\text {tso }}\left(G_{2}, P_{2}\right)$ and $\overline{p c} ; \Gamma \vdash^{\text {tso }} P_{1}$ and wellStruct $P_{1}$. Suppose also that $\left(G_{1}, P_{1}\right) \Longrightarrow{ }^{\text {tso* }}\left(G_{1}^{\prime}, P_{1}^{\prime}\right)$. Then there exists $G_{2}^{\prime}, P_{2}^{\prime}$ such that $\left(G_{1}^{\prime}, P_{1}^{\prime}\right) \sim_{\Gamma}^{\text {tso }}\left(G_{2}^{\prime}, P_{2}^{\prime}\right)$ and $\left(G_{2}, P_{2}\right) \Longrightarrow^{\text {tso* }}\left(G_{2}^{\prime}, P_{2}^{\prime}\right)$.

Proof. By finitely many application of Lemmas 5 and 12 and Theorem 1.

Corollary 2. Suppose $G_{1} \sim_{\Gamma}^{\text {tso }} G_{2}$ and $p c ; \Gamma \vdash^{\text {tso }} t$ and src t.cmd. If $\left(G_{1}, t\right) \Longrightarrow^{\text {tso* }}\left(G_{1}^{\prime}, 0\right)$ then $\left(G_{2}, t\right) \Longrightarrow{ }^{\text {tso* }}\left(G_{2}^{\prime}\right.$, o $)$ for some $G_{2}^{\prime}$ where $G_{1}^{\prime} \sim_{\Gamma}^{\text {tso }} G_{2}^{\prime}$.

Proof. An instantiation of the first corollary of Theorem 1 shows that $\left(G_{2}, t\right)$ reduces to a configuration where we may conclude with several applications of Lemma 14 and applications of EC-REAP.

Corollary 3 (TSO Simple possibilistic noninterference). Suppose $p c ; \Gamma \vdash^{\text {tso }} c$ and src $c$. Then $c$ is possibilistically noninterfering under tso and $\Gamma$.

Proof. Immediate from the second corollary of Theorem 1.

\section{Data-Race Freedom}

We want to show that our TSO and SC machines are equivalent for data-race free programs.

\subsection{SC Executions are a Subset of TSO Executions}

First, it is clear that every possibilistic SC execution is also a possibilistic TSO execution. We formalize this with the following lemma:

Lemma 41 (SC-Eval Implies TSO-Eval). If $(G, P) \Longrightarrow^{\text {sc }}\left(G^{\prime}, P^{\prime}\right)$ then $(G, P) \Longrightarrow^{\text {tso }}\left(G^{\prime}, P^{\prime}\right)$.

Proof. Since $(G, P) \Longrightarrow^{\text {sc }}\left(G^{\prime}, P^{\prime}\right)$ we have that $P=t_{1} \ldots t_{i-1}\left\|t_{i}\right\| t_{i+1} \ldots t_{n}$ and $\left(G, t_{i}\right) \longrightarrow^{o p}\left(G^{\prime}, Q\right)$ and $P^{\prime}=t_{1} \ldots t_{i-1}\|Q\| t_{i+1} \ldots t_{n}$. Therefore also $(G, P) \Longrightarrow^{\text {tso }}\left(G^{\prime}, P^{\prime}\right)$.

Corollary 4 (SC-Eval* Implies TSO-Eval*). If $(G, P) \Longrightarrow^{\text {sc* }}\left(G^{\prime}, P^{\prime}\right)$ then $(G, P) \Longrightarrow^{\text {tso* }}\left(G^{\prime}, P^{\prime}\right)$. 


\subsection{Definition of Data Race Freedom}

Definition 12 (Reads Next). Thread $\langle L, c\rangle$ reads $X$ next if one of the following conditions holds:

- $c$ has the form $x:=X$

- $c$ has the form $c_{1} ; c_{2}$ and $\left\langle L, c_{1}\right\rangle$ reads $X$ next

- $c$ has the form holding $\ell$ do $c^{\prime}$ and $\ell \in L$ and $\left\langle L, c^{\prime}\right\rangle$ reads $X$ next

Definition 13 (Writes Next). Thread $\langle L, c\rangle$ writes $X$ next if one of the following conditions holds:

- $c$ has the form $X:=x$

- $c$ has the form $c_{1} ; c_{2}$ and $\left\langle L, c_{1}\right\rangle$ writes $X$ next

- $c$ has the form holding $\ell \mathbf{d o} c^{\prime}$ and $\ell \in L$ and $\left\langle L, c^{\prime}\right\rangle$ writes $X$ next

Definition 14 (Accesses Next). Thread $t$ accesses $X$ next if either $t$ reads $X$ next or $t$ writes $X$ next.

Definition 15 (Conflicting Threads). Threads $s$ and $t$ conflict if there exists a variable $X$ such that each thread accesses $X$ next and at least one thread writes $X$ next.

Definition 16 (Race-Exhibiting Process Soup). Process soup $P$ exhibits a race if it contains two distinct threads that conflict.

Definition 17 (Race-Free Configuration). Configuration $(G, P)$ is race-free if for all $G^{\prime}$ and $P^{\prime}$ such that $(G, P) \Longrightarrow{ }^{\mathrm{sc*}}\left(G^{\prime}, P^{\prime}\right)$, it is not the case that $P^{\prime}$ exhibits a race.

\subsection{TSO Executions are a Subset of SC Executions for DRF Programs}

\subsubsection{Definitions}

It will be convenient to associate each step of computation with its action - the kind of step taken (commit or eval) and the thread that takes the step. Accordingly we annotate our evaluation steps as follows:

$$
\begin{aligned}
& o p(i) \in \operatorname{ReadySC}(\chi)
\end{aligned}
$$

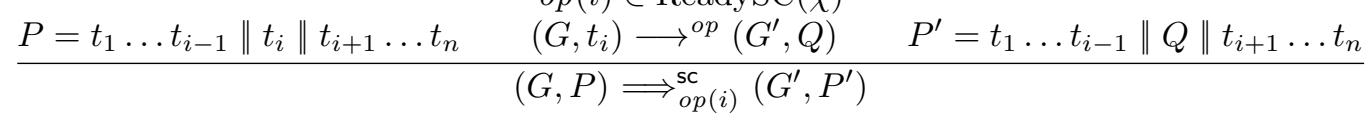

$$
\begin{aligned}
& \frac{P=t_{1} \ldots t_{i-1}\left\|t_{i}\right\| t_{i+1} \ldots t_{n} \quad\left(G, t_{i}\right) \longrightarrow^{o p}\left(G^{\prime}, Q\right) \quad P^{\prime}=t_{1} \ldots t_{i-1}\|Q\| t_{i+1} \ldots t_{n}}{(G, P) \Longrightarrow_{o p(i)}^{\text {tso }}\left(G^{\prime}, P^{\prime}\right)}
\end{aligned}
$$

Next we define an invariant on any configuration in a valid TSO execution of a program:

Definition 18. Consider configuration $G, t_{1}\|\ldots\| t_{n}$ Such a configuration is well-locked if lock sets G.locks, $t_{1}$.locks $, \ldots, t_{n}$.locks are pairwise disjoint.

Now we define an invariant on any configuration in the TSO execution of a race-free program:

Definition 19 (Well-Behaved Configuration). A configuration $(G, P)$ is well behaved if the following conditions hold, where $P=\left\langle L_{1}, c_{1}\right\rangle\|\ldots\|\left\langle L_{n}, c_{n}\right\rangle$ :

- $P$ does not exhibit a race

- $(G, P)$ is well locked

- If $L_{i}$.wb contains a write (possibly multiple) to some variable $X$, then for each $j \neq i, L_{j}$.wb does not contain a write to $X$ and $c_{j}$ does not access $X$ next.

Next we need to formalize the relationship between a TSO execution and a corresponding SC execution. The two executions will not stay in lockstep but their configurations will always have a strong relationship (if the original program is data-race-free). Let $\hat{L}$ denote the "cleared" version of $L$ - the local state identical to $L$ but with an empty write buffer. That is, $\hat{L} . m e m=L$.mem and $\hat{L}$.locks $=$ L.locks and $\hat{L} . w b=$ nil. 
Definition 20. Let $P=\left\langle L_{1}, c_{1}\right\rangle_{\iota_{1}}\|\ldots\|\left\langle L_{n}, c_{n}\right\rangle_{\iota_{n}}$. We say that $(H, Q)$ is the commit closure of $(G, P)$ if the following conditions hold:

1. $Q=\left\langle\hat{L}_{1}, c_{1}\right\rangle_{\iota_{1}}\|\ldots\|\left\langle\hat{L}_{n}, c_{n}\right\rangle_{\iota_{n}}$

2. H.locks $=$ G.locks

3. For each variable $X$ that does not appear in any write buffer in $P$, we have H.mem $(X)=$ G.mem $(X)$.

4. For each variable $X$ that appears in the write buffer of some thread $i$ in $P$, there exists a $k$ such that H.mem $(X)=k$ and $\left(G . m e m ; L_{i} . w b\right)[X] \Downarrow k$.

Intuitively, if $(H, Q)$ is the commit closure $(G, P)$ and $(G, P)$ is well behaved then $(H, Q)$ is the unique configuration that results from executing commit operations in any order from $(G, P)$ until all write buffers are empty.

\subsubsection{Simple Lemmas}

Notation: We denote by $P . l s(i)$ the local state in thread $i$ of $P$ and by $P$.cmd $(i)$ the command in thread $i$ of $P$. We denote by $P[i \mapsto t]$ the process soup identical to $P$ but with the $i$ th thread replaced by $t$. We use $P[i \mapsto L]$ as shorthand for $P[i \mapsto\langle L, P . c m d(i)\rangle]$ and $P[i \mapsto c]$ as shorthand for $P[i \mapsto\langle P . l s(i), c\rangle]$. For all of these notations, we also allow the thread to be indexed by its TID rather than its position. Finally, we use $P[i \mapsto Q]$ to denote the process soup $t_{1} \ldots t_{i-1}\|Q\| t_{i+1} \ldots t_{n}$, where $P=t_{1} \ldots t_{i-1}\left\|t_{i}\right\| t_{i+1} \ldots t_{n}$.

Lemma 42 (Preservation of well-lockedness). Suppose $(G, P)$ is well-locked and $(G, P) \Longrightarrow{ }^{\text {tso }}\left(G^{\prime}, P^{\prime}\right)$. Then $\left(G^{\prime}, P^{\prime}\right)$ is also well locked.

Lemma 43. If $(S ; W)[Y] \Downarrow j$ and $X \neq Y$, then $(S[X \mapsto k] ; W)[Y] \Downarrow j$.

Lemma 44. If $(S ;(X:=k):: W)[Y] \Downarrow j$ and $X \neq Y$, then $(S ; W)[Y] \Downarrow j$.

Lemma 45. If $(S ; W)[Y] \Downarrow j$ and $X \neq Y$, then $(S ; W+(X:=k))[Y] \Downarrow j$.

Lemma 46. If $(S ;(X:=k):: W)[X] \Downarrow j$ then $(S[X \mapsto k] . m e m ; W)[X] \Downarrow j$.

Lemma 47. If $(S ; W)[X] \Downarrow k$ and $X$ does not appear in $W$, then $S(X)=k$.

Lemma 48. If $(G, P)$ is well behaved and $(H, Q)$ is the commit closure of $(G, P)$ and P.cmd $(i)$ accesses $X$ next and $(G . m e m ; P . l s(i) . w b)[X] \Downarrow k$, then H.mem $(X)=k$.

Lemma 49. $\left\langle L, c_{1} ; c_{2}\right\rangle$ reads [writes] $X$ next if and only if $\left\langle L, c_{1}\right\rangle$ reads [writes] $X$ next.

Lemma 50. If $\langle L$, holding $\ell$ do $c\rangle$ reads [writes] $X$ next then $\langle L, c\rangle$ reads [writes] $X$ next. If $\langle L, c\rangle$ reads [writes] $X$ next then $\langle L \cup\{\ell\}$, holding $\ell$ do $c\rangle$ reads [writes] $X$ next.

Lemma 51. Suppose $(G, P)$ is well behaved, let $\langle L, c\rangle$ be the ith thread of $P$, and let $c^{\prime}$ be a command. If the set of variables that $\left\langle L, c^{\prime}\right\rangle$ reads next are a subset of those that $\langle L, c\rangle$ accesses reads next, and similarly for writes, then $\left(G, P\left[i \mapsto c^{\prime}\right]\right)$ is well behaved.

Lemma 52. If $(H, Q)$ is the commit closure of $(G, P)$, then $(H, Q[i \mapsto c])$ is the commit closure of $(G, P[i \mapsto$ $c]$ ).

Lemma 53. If $P$ exhibits a race and $(H, Q)$ is the commit closure of $(G, P)$, then $Q$ exhibits a race.

Lemma 54. If $(G,\langle L, c\rangle) \longrightarrow^{\text {eval }}\left(G^{\prime}, P^{\prime}\right)$ and $c$ has the form $\mathcal{C}[X:=x]$ then $\langle L, c\rangle$ writes $X$ next.

Notation: We denote by $P / \iota$ the process soup identical to $P$ but with thread $\iota$ removed. We denote by $G / X$ the global state identical to $G$ but with the value of $X$ set to 0 . 


\subsubsection{Key Lemmas}

Lemma 55. Suppose $(G, P)$ is well behaved and $(G, P) \Longrightarrow \underset{o p(i)}{\text { tso }}\left(G^{\prime}, P^{\prime}\right)$ and $(H, Q)$ is the commit closure of $(G, P)$.

1. If op $=$ commit then $(H, Q)$ is the commit closure of $\left(G^{\prime}, P^{\prime}\right)$.

2. If op $=$ eval and thread $t_{i}$ writes some variable $X$ next, then there exist $\left(H_{0}, Q_{o}\right)$ and $\left(H^{\prime}, Q^{\prime}\right)$ such that $(H, Q) \Longrightarrow_{o p(i)}^{\mathrm{sc}}\left(H_{0}, Q_{0}\right)$ and $\left(H_{0}, Q_{0}\right) \Longrightarrow_{\text {commit }(i)}^{\mathrm{sc}}\left(H^{\prime}, Q^{\prime}\right)$, where $\left(H^{\prime}, Q^{\prime}\right)$ is the commit closure of $\left(G^{\prime}, P^{\prime}\right)$.

3. If op $=$ eval and thread $t_{i}$ does not write any variable next, then there exists $\left(H^{\prime}, Q^{\prime}\right)$ such that $(H, Q) \Longrightarrow \underset{o p(i)}{\mathrm{sc}}\left(H^{\prime}, Q^{\prime}\right)$, where $\left(H^{\prime}, Q^{\prime}\right)$ is the commit closure of $\left(G^{\prime}, P^{\prime}\right)$.

Proof. So we have $P=t_{1} \ldots t_{i-1}\left\|t_{i}\right\| t_{i+1} \ldots t_{n}$ and $\left(G, t_{i}\right) \longrightarrow^{o p}\left(G^{\prime}, P_{0}\right)$ and $P^{\prime}=t_{1} \ldots t_{i-1}\left\|P_{0}\right\| t_{i+1} \ldots t_{n}$. Let $t_{i}=\left\langle L_{i}, c_{i}\right\rangle$.

1. Then $L_{i}$ has the form $(X:=k):: L_{0}$ and $P_{0}=\left\langle L_{0}, c_{i}\right\rangle$ and $G^{\prime}=G[X \mapsto k]$. Then $\hat{L}_{i}=\hat{L}_{0}$, so since thread $i$ is the only one that is modified from $P$ to $P^{\prime}$, condition 1 of the commit closure holds. Since locks are unchanged from $G$ to $G^{\prime}$, condition 2 holds as well.

Consider a variable $Y \neq X$. If $Y$ does not appear in any write buffer of $P$ then we have H.mem $(Y)=$ G.mem $(Y)$. By definition also $Y$ is not in any write buffer of $P^{\prime}$, and $G^{\prime}$.mem $(Y)=G$.mem $(Y)$, so condition 3 is satisfied for $Y$. If $Y$ appears in the write buffer of some thread $j$ in $P$, then H.mem $(Y)$ $=n$ and $\left(G . m e m ; L_{j} . w b\right)[Y] \Downarrow n$ for some value $n$. By definition $Y$ also appears in the write buffer of thread $j$ in $P^{\prime}$. If $j \neq i$ then by Lemma 43 we have $\left(G^{\prime} . m e m ; L_{j}\right.$.wb) $[Y] \Downarrow n$, satisfying condition 4 . If $j=i$ then by Lemmas 44 and 43 we have $\left(G^{\prime} . m e m ; L_{0} . w b\right)[Y] \Downarrow n$, satisfying condition 4 .

Finally consider variable $X$. Since $X$ is in the write buffer of $L_{i}$ we know that H.mem $(X)=n$ and $\left(G . m e m ; L_{i} . w b\right)[X] \Downarrow n$ for some $n$. Further since $(G, P)$ is well behaved we know that $X$ is not in the write buffer of any thread other than $i$ in $P$ and therefore also in $P^{\prime}$. By Lemma 46 we have $\left(G^{\prime} . m e m ; L_{0} . w b\right)[X] \Downarrow n$. If $X$ is in the write buffer of $L_{0}$ then we have satisfied condition 4 . Otherwise $X$ is not in any write buffer in $P^{\prime}$. By Lemma 47 we have that $G^{\prime}$.mem $(X)=n$, so condition 3 is satisfied for $X$.

2. We proceed by induction on the derivation of $\left(G, t_{i}\right) \longrightarrow^{o p}\left(G^{\prime}, P_{0}\right)$. Case analysis on the form of $c_{i}$, which we are given writes variable $X$ next:

$X:=x$ Then rule EC-STORE is used to step, so $L_{i}^{\prime}=L_{i}+\left(X:=L_{i}(x)\right)$ and $P_{0}=\left\langle L_{i}^{\prime}\right.$, skip $\rangle$ and $G^{\prime}=G$.

I claim that $(H, Q) \Longrightarrow_{o p(i)}^{\mathrm{sc}}\left(H_{0}, Q_{0}\right)$ and $\left(H_{0}, Q_{0}\right) \Longrightarrow_{\text {commit }(i)}^{\mathrm{sc}}\left(H^{\prime}, Q^{\prime}\right)$ for some $H_{0}, Q_{0}, H^{\prime}$, and $Q^{\prime}$. Since $(H, Q)$ is the commit closure of $(G, P)$ we know that the $i$ th thread of $(H, Q)$ is $\left\langle\hat{L}_{i}, c_{i}\right\rangle$. Further we know that all write buffers in $Q$ are empty, so no commit actions are ready on any thread. Therefore, the first step follows by EC-STORE, which then enables the commit step. By definition $\hat{L}_{i} \cdot m e m=L_{i}$. mem, so $\hat{L}_{i}(x)=L_{i}(x)$, so $H^{\prime}=H\left[X \mapsto L_{i}(x)\right]$. Also, $Q^{\prime}$ is identical to $Q$ except the $i$ th thread's command is skip.

We now prove that $\left(H^{\prime}, Q^{\prime}\right)$ is the commit closure of $\left(G^{\prime}, P^{\prime}\right)$. Condition 1 holds for all threads other than $i$, since these threads are unchanged from $Q$ and $P$ and we have that $(H, Q)$ is the commit closure of $(G, P)$. For thread $i$ the result follows by definition of these threads in $P^{\prime}$ and $Q^{\prime}$ and the fact that $\hat{L}_{i}$ is also the "cleared" version of $L_{i}+\left(X:=L_{i}(x)\right)$. Condition 2 follows from the fact that locks are unchanged from $G$ to $G^{\prime}$ and $H$ to $H^{\prime}$.

For Condition 3, let $S$ be the set of variables that do not appear in any write buffer in $P$. Then $S-\{X\}$ is the set of variables that do not appear in any write buffer in $P^{\prime}$. For each such variable $Y, G(Y)=G^{\prime}(Y)$ and $H(Y)=H^{\prime}(Y)$ by the definition of $G^{\prime}$ and $H^{\prime}$. Then the result follows from the fact that $G(Y)=H(Y)$.

For Condition 4, first consider a variable $Y \neq X$ that appears in the write buffer of some thread $j$ in $P^{\prime}$. Then it also appears in the write buffer of thread $j$ in $P$, so we know that H.mem $(Y)$ $=k$ and $\left(G\right.$.mem; $\left.L_{j} . w b\right)[Y] \Downarrow k$, where $L_{j}$ is the local state of thread $j$ in $P$. Then by definition of $H^{\prime}$ also $H^{\prime}$. mem $(Y)=k$, and since $G^{\prime}=G$ we have $\left(G^{\prime}\right.$.mem; $L_{j}$.wb) $[Y] \Downarrow k$. If $j \neq i$ we are done; otherwise by Lemma 45 we have $\left(G^{\prime} . m e m ; L_{i}^{\prime}\right.$.wb) $[Y] \Downarrow k$. 
Finally consider variable $X$. Since $t_{i}$ writes $X$ next and $(G, P)$ is well behaved, we know that $X$ is not in the write buffer of any thread other than $i$ in $P$, and hence also in $P^{\prime}$. By the definition of $L_{i}^{\prime}$ and the lookup procedure we have that $\left(G^{\prime}\right.$.mem; $L_{i}^{\prime}$.wb) $[X] \Downarrow L_{i}(x)$. By definition of $H^{\prime}$ we have $H^{\prime}(X)=L_{i}(x)$ so the result follows.

$c_{i 1}$; $c_{i 2}$ Since $\left\langle L_{i}, c_{i 1}\right\rangle$ writes $X$ next we know that $c_{i 1}$ is not skip, so the step must occur with rule ECSeqStruct. Therefore we have $\left(G,\left\langle L_{i}, c_{i 1}\right\rangle\right) \longrightarrow^{\text {eval }}\left(G^{\prime},\left\langle L_{i}^{\prime}, c_{i 1}^{\prime}\right\rangle \| P_{0}^{\prime}\right)$ and $P_{0}=\left\langle L_{i}^{\prime}, c_{i 1}^{\prime} ; c_{i 2}\right\rangle \| P_{0}^{\prime}$. Therefore we have $\left(G, P\left[i \mapsto c_{i 1}\right]\right) \Longrightarrow_{o p(i)}^{\text {tso }}\left(G^{\prime}, P^{\prime}\left[i \mapsto c_{i 1}^{\prime}\right]\right)$. By Lemma 49 the set of variables that $\left\langle L_{i}, c_{i 1}\right\rangle$ reads [writes] next are a subset of those that $\left\langle L_{i}, c_{i 1} ; c_{i 2}\right\rangle$ reads [writes] next. Therefore by Lemma 51 we have that $\left(G, P\left[i \mapsto c_{i 1}\right]\right)$ is well behaved. By Lemma $52\left(H, Q\left[i \mapsto c_{i 1}\right]\right)$ is the commit closure of $\left(G, P\left[i \mapsto c_{i 1}\right]\right)$. Finally by Lemma 49 we know that $\left\langle L_{i}, c_{i 1}\right\rangle$ writes $X$ next.

Therefore by induction there exist $\left(H_{0}, Q_{o}\right)$ and $\left(H^{\prime}, Q^{\prime}\right)$ such that $\left(H, Q\left[i \mapsto c_{i 1}\right]\right) \Longrightarrow_{o p(i)}^{\mathrm{sc}}\left(H_{0}, Q_{0}\right)$ and $\left(H_{0}, Q_{0}\right) \Longrightarrow{ }_{\text {commit }(i)}^{\mathrm{sc}}\left(H^{\prime}, Q^{\prime}\right)$, where $\left(H^{\prime}, Q^{\prime}\right)$ is the commit closure of $\left(G^{\prime}, P^{\prime}\left[i \mapsto c_{i 1}^{\prime}\right]\right)$. Then by rule EC-SEQSTRUCT it follows that

$$
(H, Q) \Longrightarrow \underset{o p(i)}{\mathrm{sc}}\left(H_{0}, Q_{0}\left[i \mapsto Q_{0} . c m d(i) ; c_{i 2}\right]\right)
$$

and by the commit rule

$$
\left(H_{0}, Q_{0}\left[i \mapsto Q_{0} . c m d(i) ; c_{i 2}\right]\right) \Longrightarrow{ }_{c o m m i t(i)}^{\mathrm{sc}}\left(H^{\prime}, Q^{\prime}\left[i \mapsto Q_{0} . c m d(i) ; c_{i 2}\right]\right)
$$

Since $\left(H^{\prime}, Q^{\prime}\right)$ is the commit closure of $\left(G^{\prime}, P^{\prime}\left[i \mapsto c_{i 1}^{\prime}\right]\right)$ we have that $Q_{0} . c m d(i)=Q^{\prime} . c m d(i)=$ $c_{i 1}^{\prime}$, so by Lemma 52 we have that $\left(H^{\prime}, Q^{\prime}\left[i \mapsto Q_{0} . c m d(i) ; c_{i 2}\right]\right)$ is the commit closure of $\left(G^{\prime}, P^{\prime}\right)$.

holding $\ell$ do $c_{i 0}$ Since $\left\langle L_{i}, c_{i}\right\rangle$ writes $X$ next in $P$ we have that $\ell \in L_{i}$ and $\left\langle L_{i}, c_{i 0}\right\rangle$ writes $X$ next. Therefore we know that $c_{i 0}$ is not skip, so the step cannot occur with rule EC-HoLdRELEASE. Therefore the step must occur with rule EC-HoldStep. Therefore we have $\left(G,\left\langle L_{i}, c_{i 0}\right\rangle\right) \longrightarrow{ }^{\text {eval }}$ $\left(G^{\prime},\left\langle L_{i}^{\prime}, c_{i 0}^{\prime}\right\rangle \| P_{0}^{\prime}\right)$ and $P_{0}=\left\langle L_{i}^{\prime}\right.$, holding $\ell$ do $\left.c_{i 0}^{\prime}\right\rangle \| P_{0}^{\prime}$. Therefore we have

$$
\left(G, P\left[i \mapsto c_{i 0}\right]\right) \Longrightarrow{ }_{o p(i)}^{\text {tso }}\left(G^{\prime}, P^{\prime}\left[i \mapsto c_{i 0}^{\prime}\right]\right)
$$

By Lemma 50 the set of variables that $\left\langle L_{i}, c_{i 0}\right\rangle$ reads [writes] next are a subset of those that $\left\langle L_{i}\right.$, holding $\ell$ do $\left.c_{i 0}\right\rangle$ reads [writes] next. Therefore by Lemma 51 we have that $\left(G, P\left[i \mapsto c_{i 0}\right]\right)$ is well behaved. By Lemma $52\left(H, Q\left[i \mapsto c_{i 0}\right]\right)$ is the commit closure of $\left(G, P\left[i \mapsto c_{i 0}\right]\right)$. Finally by Lemma 50 we know that $\left\langle L_{i}, c_{i 0}\right\rangle$ accesses $X$ next.

Therefore by induction there exist $\left(H_{0}, Q_{o}\right)$ and $\left(H^{\prime}, Q^{\prime}\right)$ such that $\left(H, Q\left[i \mapsto c_{i 0}\right]\right) \Longrightarrow_{o p(i)}^{\mathrm{sc}}\left(H_{0}, Q_{0}\right)$ and $\left(H_{0}, Q_{0}\right) \Longrightarrow{ }_{\text {commit }(i)}^{\text {sc }}\left(H^{\prime}, Q^{\prime}\right)$, where $\left(H^{\prime}, Q^{\prime}\right)$ is the commit closure of $\left(G^{\prime}, P^{\prime}\left[i \mapsto c_{i 0}^{\prime}\right]\right)$. Since $\ell \in L_{i}$ also $\ell \in \hat{L}_{i}$, so by rule EC-HoLDSTEP it follows that

$$
(H, Q) \Longrightarrow{ }_{o p(i)}^{\mathrm{sc}}\left(H_{0}, Q_{0}\left[i \mapsto \text { holding } \ell \text { do } Q_{0} . c m d(i)\right]\right)
$$

and by the commit rule

$$
\left(H_{0}, Q_{0}\left[i \mapsto \text { holding } \ell \text { do } Q_{0} . c m d(i)\right]\right) \Longrightarrow \underset{c o m m i t(i)}{\text { sc }}\left(H^{\prime}, Q^{\prime}\left[i \mapsto \text { holding } \ell \text { do } Q_{0} . c m d(i)\right]\right)
$$

Since $\left(H^{\prime}, Q^{\prime}\right)$ is the commit closure of $\left(G^{\prime}, P^{\prime}\left[i \mapsto c_{i 0}^{\prime}\right]\right)$, we have $Q_{0} \cdot \operatorname{cmd}(i)=Q^{\prime} \cdot \operatorname{cmd}(i)=c_{i 0}^{\prime}$, so by Lemma 52 we have that $\left(H^{\prime}, Q^{\prime}\left[i \mapsto\right.\right.$ holding $\ell$ do $\left.\left.Q_{0} . c m d(i)\right]\right)$ is the commit closure of $\left(G^{\prime}, P^{\prime}\right)$.

3. We proceed by induction on the derivation of $\left(G, t_{i}\right) \longrightarrow^{o p}\left(G^{\prime}, P_{0}\right)$. Case analysis of the rule used to take this step:

EC-LOAD Then $c_{i}$ has the form $x:=X$ and $\left(G . m e m ; L_{i} . w b\right)[X] \Downarrow n$ and $P_{0}=\left\langle L_{i}[x \mapsto n]\right.$, skip $\rangle$ and $G^{\prime}=G$. Since $c_{i}$ accesses $X$ next, by Lemma 48 we have $H \cdot m e m(X)=n$, and since $\hat{L}_{i}$ has an empty write buffer also $\left(H . m e m ; \hat{L}_{i} . w b\right)[X] \Downarrow n$. Then by EC-LOAD we have $\left(H,\left\langle\hat{L}_{i}, c_{i}\right\rangle\right) \longrightarrow{ }^{o p}$ $\left(H, Q_{0}\right)$, where $Q_{0}=\left\langle\hat{L}_{i}[x \mapsto n]\right.$, skip $\rangle$. Therefore $(H, Q) \Longrightarrow_{o p(i)}^{\mathrm{sc}}\left(H^{\prime}, Q^{\prime}\right)$, where $H^{\prime}=H$ and $Q^{\prime}=Q\left[i \mapsto Q_{0}\right]$

Now we argue that $\left(H^{\prime}, Q^{\prime}\right)$ is the commit closure of $\left(G^{\prime}, P^{\prime}\right)$. Since $\hat{L}_{i}[x \mapsto n]$ is the "cleared" version of $L_{i}[x \mapsto n]$, condition 1 holds for thread $i$, and it continues to hold for all other threads, which are unchanged. Condition 2 continues to hold since locks are not changed. Since $G^{\prime}=G$ and $H^{\prime}=H$ and no write buffers are modified, conditions 3 and 4 continue to hold. 
EC-EvalExP Then $c_{i}$ has the form $x:=a$ and $L_{i}[a] \Downarrow n$ and $P_{0}=\left\langle L_{i}[x \mapsto n]\right.$, skip $\rangle$ and $G^{\prime}=G$. By definition of $\hat{L}_{i}$ also $\hat{L}_{i}[a] \Downarrow n$, so by EC-EvaLExP we have $\left(H,\left\langle\hat{L}_{i}, c_{i}\right\rangle\right) \longrightarrow^{o p}\left(H, Q_{0}\right)$, where $Q_{0}=\left\langle\hat{L}_{i}[x \mapsto n]\right.$, skip $\rangle$. Therefore $(H, Q) \Longrightarrow \underset{o p(i)}{\mathrm{sc}}\left(H^{\prime}, Q^{\prime}\right)$, where $H^{\prime}=H$ and $Q^{\prime}=Q\left[i \mapsto Q_{0}\right]$. Finally, we can argue that $\left(H^{\prime}, Q^{\prime}\right)$ is the commit closure of $\left(G^{\prime}, P^{\prime}\right)$ as in the case for EC-LOAD above.

EC-SYNCACQUiRe Then $c_{i}$ has the form sync $\ell$ do $c_{i 0}$ and $\ell \in G$ and $L_{i} . w b=n i l$ and $G^{\prime}=G \backslash\{\ell\}$ and $P_{0}=\left\langle L_{i} \cup\{\ell\}\right.$, holding $\ell$ do $\left.c_{i 0}\right\rangle$. Since $(H, Q)$ is the commit closure of $(G, P)$, also $\ell \in H$, and by definition $\hat{L}_{i}$. wb $=$ nil. Therefore by EC-SYNCACQUIRE we have $\left(H,\left\langle\hat{L}_{i}, c_{i}\right\rangle\right) \longrightarrow{ }^{o p}\left(H^{\prime}, Q_{0}\right)$, where $H^{\prime}=H \backslash\{\ell\}$ and $Q_{0}=\left\langle\hat{L}_{i} \cup\{\ell\}\right.$, holding $\ell$ do $\left.c_{i 0}\right\rangle$. Therefore $(H, Q) \Longrightarrow_{o p(i)}^{\mathrm{sc}}\left(H^{\prime}, Q^{\prime}\right)$, where $Q^{\prime}=Q\left[i \mapsto Q_{0}\right]$.

Now we argue that $\left(H^{\prime}, Q^{\prime}\right)$ is the commit closure of $\left(G^{\prime}, P^{\prime}\right)$. Since $\hat{L}_{i} \cup\{\ell\}$ is also the "cleared" version of $L_{i} \cup\{\ell\}$, condition 1 holds for thread $i$, and it continues to hold for all other threads, which are unchanged. Condition 2 continues to hold since both $G$ and $H$ get $\ell$ removed from their locksets. Since $G^{\prime}$. mem $=$ G.mem and $H^{\prime}$.mem $=H$.mem and no write buffers are modified, conditions 3 and 4 continue to hold.

EC-SyncReenter Then $c_{i}$ has the form sync $\ell$ do $c_{i 0}$ and $\ell \in L_{i}$ and $G^{\prime}=G$ and $P_{0}=\left\langle L_{i}, c_{i 0}\right\rangle$. Therefore also $\ell \in \hat{L}_{i}$, so by EC-SYNCREEnTER we have $\left(H,\left\langle\hat{L}_{i}, c_{i}\right\rangle\right) \longrightarrow^{o p}\left(H, Q_{0}\right)$, where $Q_{0}=$ $\left\langle\hat{L}_{i}\right.$, fence; $\left(c_{i 0} ;\right.$ fence $\left.)\right\rangle$. Therefore $(H, Q) \Longrightarrow_{o p(i)}^{\mathrm{sc}}\left(H^{\prime}, Q^{\prime}\right)$, where $H^{\prime}=H$ and $Q^{\prime}=Q[i \mapsto$ fence; $\left(c_{i 0}\right.$; fence) $]$. Then by Lemma 52 we have that $\left(H^{\prime}, Q^{\prime}\right)$ is the commit closure of $\left(G^{\prime}, P^{\prime}\right)$.

EC-HoldSteP Then $c_{i}$ has the form holding $\ell$ do $c_{i 0}$ and $\ell \in L_{i}$ and $\left(G,\left\langle L_{i}, c_{i 0}\right\rangle\right) \longrightarrow{ }^{\text {eval }}\left(G^{\prime},\left\langle L_{i}^{\prime}, c_{i 0}^{\prime}\right\rangle \| P_{0}^{\prime}\right)$ and $P_{0}=\left\langle L_{i}^{\prime}\right.$, holding $\left.\ell \mathbf{d o} c_{i 0}^{\prime}\right\rangle \| P_{0}^{\prime}$. Therefore we have $\left(G, P\left[i \mapsto c_{i 0}\right]\right) \Longrightarrow_{o p(i)}^{\text {tso }}\left(G^{\prime}, P^{\prime}\left[i \mapsto c_{i 0}^{\prime}\right]\right)$. By Lemma 50 the set of variables that $\left\langle L_{i}, c_{i 0}\right\rangle$ reads [writes] next are a subset of those that $\left\langle L_{i}\right.$, holding $\ell$ do $\left.c_{i 0}\right\rangle$ reads [writes] next. Therefore by Lemma 51 we have that $\left(G, P\left[i \mapsto c_{i 0}\right]\right)$ is well behaved. Then by Lemma $52\left(H, Q\left[i \mapsto c_{i 0}\right]\right)$ is the commit closure of $\left(G, P\left[i \mapsto c_{i 0}\right]\right)$. Finally by Lemma 50 we know that $\left\langle L_{i}, c_{i 0}\right\rangle$ does not write any variables next.

Therefore by induction there exist $\left(H^{\prime}, Q^{\prime}\right)$ such that $\left(H, Q\left[i \mapsto c_{i 0}\right]\right) \Longrightarrow_{o p(i)}^{\mathrm{sc}}\left(H^{\prime}, Q^{\prime}\right)$, where $\left(H^{\prime}, Q^{\prime}\right)$ is the commit closure of $\left(G^{\prime}, P^{\prime}\left[i \mapsto c_{i 0}^{\prime}\right]\right)$. Since $\ell \in L_{i}$ also $\ell \in \hat{L}_{i}$. Then by rule EC-HoLdSTEP it follows that

$$
(H, Q) \Longrightarrow \underset{o p(i)}{\mathrm{sc}}\left(H^{\prime}, Q^{\prime}\left[i \mapsto \text { holding } \ell \text { do } Q^{\prime} . c m d(i)\right]\right)
$$

Since $\left(H^{\prime}, Q^{\prime}\right)$ is the commit closure of $\left(G^{\prime}, P^{\prime}\left[i \mapsto c_{i 0}^{\prime}\right]\right)$ we have that $Q^{\prime} . c m d(i)=c_{i 0}^{\prime}$, so by Lemma 52 we have that $\left(H^{\prime}, Q^{\prime}\left[i \mapsto\right.\right.$ holding $\ell$ do $\left.\left.Q^{\prime} . c m d(i)\right]\right)$ is the commit closure of $\left(G^{\prime}, P^{\prime}\right)$.

EC-HoldRelease Then $c_{i}$ has the form holding $\ell$ do skip and $\ell \in L_{i}$ and $L_{i}$.wb $=$ nil and $G^{\prime}=$ $G \cup\{\ell\}$ and $P_{0}=\left\langle L_{i} \backslash\{\ell\}\right.$, skip $\rangle$. Then by definition $\ell \in \hat{L}_{i}$ and $\hat{L}_{i} \cdot w b=$ nil. Therefore by EC-HoldRelease we have $\left(H,\left\langle\hat{L}_{i}, c_{i}\right\rangle\right) \longrightarrow^{o p}\left(H^{\prime}, Q_{0}\right)$, where $H^{\prime}=H \cup\{\ell\}$ and $Q_{0}=$ $\left\langle\hat{L}_{i} \backslash\{\ell\}\right.$, skip $\rangle$. Therefore $(H, Q) \Longrightarrow_{o p(i)}^{\mathrm{sc}}\left(H^{\prime}, Q^{\prime}\right)$, where $Q^{\prime}=Q\left[i \mapsto Q_{0}\right]$.

Now we argue that $\left(H^{\prime}, Q^{\prime}\right)$ is the commit closure of $\left(G^{\prime}, P^{\prime}\right)$. Since $\hat{L}_{i} \backslash\{\ell\}$ is also the "cleared" version of $L_{i} \backslash\{\ell\}$, condition 1 holds for thread $i$, and it continues to hold for all other threads, which are unchanged. Condition 2 continues to hold since both $G$ and $H$ get $\ell$ added to their locksets. Since $G^{\prime}$. mem $=$ G.mem and $H^{\prime}$. mem $=H . m e m$ and no write buffers are modified, conditions 3 and 4 continue to hold.

EC-Fence Then $c_{i}$ has the form fence and $L_{i} \cdot w b=n i l$ and $G^{\prime}=G$ and $P_{0}=\left\langle L_{i}\right.$, skip $\rangle$. By definition $\hat{L}_{i} . w b=n i l$. Therefore by EC-Fence we have $\left(H,\left\langle\hat{L}_{i}, c_{i}\right\rangle\right) \longrightarrow^{o p}\left(H, Q_{0}\right)$, where $Q_{0}=\left\langle\hat{L}_{i}\right.$, skip $\rangle$. Therefore $(H, Q) \Longrightarrow{ }_{o p(i)}^{\mathrm{sc}}\left(H^{\prime}, Q^{\prime}\right)$, where $H^{\prime}=H$ and $Q^{\prime}=Q\left[i \mapsto Q_{0}\right]$. Then by Lemma 52 we have that $\left(H^{\prime}, Q^{\prime}\right)$ is the commit closure of $\left(G^{\prime}, P^{\prime}\right)$.

EC-FORK Then $c_{i}$ has the form fork $c_{i 0}$ and $L_{i}$.wb $=$ nil and $G^{\prime}=G$ and $P_{0}=\left\langle L_{i}\right.$, skip $\rangle \|\left\langle L_{\oslash}, c_{i 0}\right\rangle$. By definition $\hat{L}_{i} \cdot w b=n i l$. Therefore by EC-Fork we have $\left(H,\left\langle\hat{L}_{i}, c_{i}\right\rangle\right) \longrightarrow^{o p}\left(H, Q_{0}\right)$, where $Q_{0}=\left\langle\hat{L}_{i}\right.$, skip $\rangle \|\left\langle L_{\oslash}, c_{i 0}\right\rangle$. Therefore $(H, Q) \underset{o p(i)}{\mathrm{sc}_{0}}\left(H^{\prime}, Q^{\prime}\right)$, where $H^{\prime}=H$ and $Q^{\prime}=Q[i \mapsto$ $\left.Q_{0}\right]$.

Now we argue that $\left(H^{\prime}, Q^{\prime}\right)$ is the commit closure of $\left(G^{\prime}, P^{\prime}\right)$. Since all local states are unchanged and $L_{\oslash}$ is its own "cleared" version by definition, condition 1 continues to hold. Condition 2 continues to hold since $G=G^{\prime}$ and $H=H^{\prime}$. Finally, since $G^{\prime}$.mem $=G$.mem and $H^{\prime}$.mem $=$ H.mem, no write buffers are modified, and $L_{\oslash}$ has an empty write buffer, conditions 3 and 4 continue to hold. 
EC-SeqStruct Then $c_{i}$ has the form $c_{i 1} ; c_{i 2}$ and $\left(G,\left\langle L_{i}, c_{i 1}\right\rangle\right) \longrightarrow^{\text {eval }}\left(G^{\prime},\left\langle L_{i}^{\prime}, c_{i 1}^{\prime}\right\rangle \| P_{0}^{\prime}\right)$ and $P_{0}=$ $\left\langle L_{i}^{\prime}, c_{i 1}^{\prime} ; c_{i 2}\right\rangle \| P_{0}^{\prime}$. Therefore we have $\left(G, P\left[i \mapsto c_{i 1}\right]\right) \Longrightarrow_{o p(i)}^{\text {tso }}\left(G^{\prime}, P^{\prime}\left[i \mapsto c_{i 1}^{\prime}\right]\right)$. By Lemma 49 the set of variables that $\left\langle L_{i}, c_{i 1}\right\rangle$ reads [writes] next are a subset of those that $\left\langle L_{i}, c_{i 1} ; c_{i 2}\right\rangle$ reads [writes] next. Therefore by Lemma 51 we have that $\left(G, P\left[i \mapsto c_{i 1}\right]\right)$ is well behaved. By Lemma 52 $\left(H, Q\left[i \mapsto c_{i 1}\right]\right)$ is the commit closure of $\left(G, P\left[i \mapsto c_{i 1}\right]\right)$. Finally by Lemma 49 we know that $\left\langle L_{i}, c_{i 1}\right\rangle$ does not write any variables next.

Therefore by induction there exist $\left(H^{\prime}, Q^{\prime}\right)$ such that $\left(H, Q\left[i \mapsto c_{i 1}\right]\right) \Longrightarrow_{o p(i)}^{\mathrm{sc}}\left(H^{\prime}, Q^{\prime}\right)$, where $\left(H^{\prime}, Q^{\prime}\right)$ is the commit closure of $\left(G^{\prime}, P^{\prime}\left[i \mapsto c_{i 1}^{\prime}\right]\right)$. Then by rule EC-SEQStruct it follows that

$$
(H, Q) \Longrightarrow \underset{o p(i)}{\mathrm{sc}}\left(H^{\prime}, Q^{\prime}\left[i \mapsto Q^{\prime} . c m d(i) ; c_{i 2}\right]\right)
$$

Since $\left(H^{\prime}, Q^{\prime}\right)$ is the commit closure of $\left(G^{\prime}, P^{\prime}\left[i \mapsto c_{i 1}^{\prime}\right]\right)$ we have that $Q^{\prime} . c m d(i)=c_{i 1}^{\prime}$, so by Lemma 52 we have that $\left(H^{\prime}, Q^{\prime}\left[i \mapsto Q^{\prime} . c m d(i) ; c_{i 2}\right]\right)$ is the commit closure of $\left(G^{\prime}, P^{\prime}\right)$.

EC-SEQSkIP Then $c_{i}$ has the form skip; $c_{i 0}$ and $G^{\prime}=G$ and $P_{0}=\left\langle L_{i}, c_{i 0}\right\rangle$. Therefore by EC-SEQSkIP we have $\left(H,\left\langle\hat{L}_{i}, c_{i}\right\rangle\right) \longrightarrow^{o p}\left(H, Q_{0}\right)$, where $Q_{0}=\left\langle\hat{L}_{i}, c_{i 0}\right\rangle$. Therefore $(H, Q) \Longrightarrow_{o p(i)}^{\mathrm{sc}}\left(H^{\prime}, Q^{\prime}\right)$, where $H^{\prime}=H$ and $Q^{\prime}=Q\left[i \mapsto Q_{0}\right]$. Then by Lemma 52 we have that $\left(H^{\prime}, Q^{\prime}\right)$ is the commit closure of $\left(G^{\prime}, P^{\prime}\right)$.

EC-IfTRue Then $c_{i}$ has the form if $b$ do $c_{i 1}$ else $c_{i 2}$ and $L_{i}[b] \Downarrow$ true and $G^{\prime}=G$ and $P_{0}=\left\langle L_{i}, c_{i 1}\right\rangle$. By definition of $\hat{L}_{i}$ also $\hat{L}_{i}[b] \Downarrow$ true, so by EC-IFTRUe we have $\left(H,\left\langle\hat{L}_{i}, c_{i}\right\rangle\right) \longrightarrow^{o p}\left(H, Q_{0}\right)$, where $Q_{0}=\left\langle\hat{L}_{i}, c_{i 1}\right\rangle$. Therefore $(H, Q) \Longrightarrow_{o p(i)}^{\mathrm{sc}}\left(H^{\prime}, Q^{\prime}\right)$, where $H^{\prime}=H$ and $Q^{\prime}=Q\left[i \mapsto Q_{0}\right]$. Then by Lemma 52 we have that $\left(H^{\prime}, Q^{\prime}\right)$ is the commit closure of $\left(G^{\prime}, P^{\prime}\right)$.

eC-IfFalse, eC-WhileTrue, and ec-WhileFalse Follows from the same argument as for rule ECIFTRUE above.

EC-REAP Then $c_{i}$ has the form skip and $L_{i}$.wb $=$ nil and $L_{i}$.locks $=\emptyset$ and $G^{\prime}=G$ and $P_{0}=$ o. Therefore by EC-REAP we have $\left(H,\left\langle\hat{L}_{i}, c_{i}\right\rangle\right) \longrightarrow^{o p}\left(H, Q_{0}\right)$, where $Q_{0}=0$. Therefore $(H, Q) \Longrightarrow_{o p(i)}^{\mathrm{sc}}\left(H^{\prime}, Q^{\prime}\right)$, where $H^{\prime}=H$ and $Q^{\prime}=Q[i \mapsto \mathrm{o}]$.

Now we argue that $\left(H^{\prime}, Q^{\prime}\right)$ is the commit closure of $\left(G^{\prime}, P^{\prime}\right)$. Condition 1 continues to hold for all threads other than $i$, which are unchanged, and thread $i$ is removed from both $P$ and $Q$. Condition 2 continues to hold since locks are not changed in $G$ or $H$. For condition 3, since $L_{i}$. wb $=$ nil we know that the set of variables that do not appear in any write buffer in $P$ is equivalent to the set that do not appear in any write buffer in $P^{\prime}$. Then since $G^{\prime}=G$ and $H^{\prime}=H$ condition 3 continues to hold. For condition 4 , again since $L_{i} . w b=n i l$ we know that a variable $X$ appears in the write buffer for some thread $j$ in $P$ if and only if it appears in that thread's write buffer in $P^{\prime}$, and furthermore $j \neq i$. Then again since $G^{\prime}=G$ and $H^{\prime}=H$ and no write buffers are modified, condition 4 continues to hold.

Lemma 56. Suppose $\left(G,\left\langle L_{\oslash}, c\right\rangle\right) \Longrightarrow^{\text {tso }}\left(G_{1}, P_{1}\right) \Longrightarrow^{\text {tso }} \ldots \Longrightarrow^{\text {tso }}\left(G_{n}, P_{n}\right) \Longrightarrow^{\text {tso }}\left(G^{\prime}, P^{\prime}\right)$ for some $n \geq 0$ and $\left(G_{k}, P_{k}\right)$ is well behaved for each $1 \leq k \leq n$ and $\left(G^{\prime}, P^{\prime}\right)$ is not well behaved. Then $\left(G,\left\langle L_{\oslash}, c\right\rangle\right)$ is not race-free.

Proof. By Definition 19 we have that $\left(G,\left\langle L_{\oslash}, c\right\rangle\right)$ is well behaved, and by the definition of $L_{\oslash}$ as well as Definition 20 also $\left(G,\left\langle L_{\oslash}, c\right\rangle\right)$ is its own commit closure. Therefore by Lemma 55 we have $\left(G,\left\langle L_{\oslash}, c\right\rangle\right) \Longrightarrow^{\text {sc* }}\left(H_{1}, Q_{1}\right)$ $\Longrightarrow^{\text {sc* }} \ldots \Longrightarrow^{\text {sc* }}\left(H_{n}, Q_{n}\right) \Longrightarrow^{\text {sc* }}\left(H^{\prime}, Q^{\prime}\right)$ where for each $1 \leq k \leq n$ we have that $\left(H_{k}, Q_{k}\right)$ is the commit closure of $\left(G_{k}, P_{k}\right)$ and $\left(H^{\prime}, Q^{\prime}\right)$ is the commit closure of $\left(G^{\prime}, P^{\prime}\right)$.

Since $\left(G^{\prime}, P^{\prime}\right)$ is not well behaved, by Definition 19 there are four possibilities. First suppose $P^{\prime}$ exhibits a race. Then by Lemma 53 so does $Q^{\prime}$ and we have shown that $\left(G,\left\langle L_{\oslash}, c\right\rangle\right)$ is not race-free. Second suppose $\left(G^{\prime}, P^{\prime}\right)$ is well locked. But since $\left(G_{n}, P_{n}\right)$ is well behaved it is also well locked, so by Lemma 42 so is $\left(G^{\prime}, P^{\prime}\right)$ and we have a contradiction. Third suppose there are distinct threads in $P^{\prime}$ that both contain a write to some variable $X$ in their write buffers. Since $\left(G_{n}, P_{n}\right)$ is well behaved we know that this is not also true in $P_{n}$. Then there must exist distinct threads $\iota$ and $\iota^{\prime}$ in $P_{n}$ such that there is a write to $X$ in $P_{n} . l s(\iota) . w b$ and $P_{n} . c m d\left(\iota^{\prime}\right)$ has the form $\mathcal{C}[X:=x]$. Then by Lemma 54 thread $\iota^{\prime}$ in $P_{n}$ writes $X$ next, so it also accesses $X$ next. But then $\left(G_{n}, P_{n}\right)$ is not well behaved, and we have a contradiction.

Finally suppose there are distinct threads $\iota$ and $\iota^{\prime}$ in $P^{\prime}$ such that there is a write to $X$ in $P^{\prime}$.ls $(\iota) . w b$ and $\iota^{\prime}$ accesses $X$ next in $P^{\prime}$. I claim that the step from $\left(G_{n}, P_{n}\right)$ to $\left(G^{\prime}, P^{\prime}\right)$ must execute an eval step on 
thread $\iota^{\prime}$, and hence also the step(s) from $\left(H_{n}, Q_{n}\right)$ to $\left(H^{\prime}, Q^{\prime}\right)$ must execute thread $\iota^{\prime}$ by the construction in Lemma 55. If the step from $\left(G_{n}, P_{n}\right)$ to $\left(G^{\prime}, P^{\prime}\right)$ executes a thread other than $\iota$ or $\iota^{\prime}$ or executes a commit step on $\iota^{\prime}$, then there is a write to $X$ in $P_{n} . l s(\iota) . w b$ and $\iota^{\prime}$ accesses $X$ next in $P_{n}$, contradicting the well-behavedness of $\left(G_{n}, P_{n}\right)$. If the step executes thread $\iota$, then again $\iota^{\prime}$ accesses $X$ next in $P_{n}$, and either there is a write to $X$ in $P_{n} . l s(\iota) . w b$ or $\iota$ writes $X$ next in $P_{n}$. Either way, the well-behavedness of $\left(G_{n}, P_{n}\right)$ is contradicted.

Let $k$ be the greatest index such that $P_{k} \cdot \operatorname{cmd}(\iota)$ has the form $\mathcal{C}[X:=x]$, the step from $\left(G_{k}, P_{k}\right)$ in our execution sequence is on thread $\iota$, and for all indices $m$ such that $k<m \leq n$ there is a write to $X$ in $P_{m} . l s(\iota) . w b$. It's clear one such process soup exists, in order to take the step that puts $X$ in the write buffer of thread $\iota$ in $P^{\prime}$. (Further, the latest such process soup cannot be the very first one, $\left\langle L_{\oslash}, c\right\rangle$, since there is only one thread initially, and forking another thread requires that the write buffer be empty.) Since $\left(H_{k}, Q_{k}\right)$ is the commit closure of $\left(G_{k}, P_{k}\right)$ also $Q_{k} \cdot \operatorname{cmd}(\iota)$ has the form $\mathcal{C}[X:=x]$.

Consider the sequence of $\Longrightarrow$ sc steps from $\left(H_{k}, Q_{k}\right)$ to $\left(H^{\prime}, Q^{\prime}\right)$, and let's rename them as follows: $\left(H_{1}^{\prime}, Q_{1}^{\prime}\right) \Longrightarrow_{o p_{1}\left(i_{1}\right)}^{\mathrm{sc}}\left(H_{2}^{\prime}, Q_{2}^{\prime}\right) \Longrightarrow^{\mathrm{sc}} \ldots \Longrightarrow^{\mathrm{sc}}\left(H_{m}^{\prime}, Q_{m}^{\prime}\right) \Longrightarrow_{o p_{m}\left(i_{m}\right)}^{\mathrm{sc}}\left(H^{\prime}, Q^{\prime}\right)$. Call the associated sequence of actions $\bar{\alpha}$, and let $\overline{\alpha_{0}}$ be identical to $\bar{\alpha}$ but with each action on thread $\iota$ removed. I claim that $\overline{\alpha_{0}}$ is also a valid SC execution sequence from $\left(H_{1}^{\prime}, Q_{1}^{\prime}\right)$ and furthermore that this sequence ends in a state $\left(H^{\prime \prime}, Q^{\prime \prime}\right)$ such that $Q^{\prime} / \iota=Q^{\prime \prime} / \iota$. If we can argue this, then we have shown that $\left(G,\left\langle L_{\oslash}, c\right\rangle\right)$ is not race-free, since in the final process soup $Q^{\prime \prime}$ of our execution sequence $\overline{\alpha_{0}}$ we have that $\iota$ writes $X$ next (because that thread is unchanged from $Q_{k}$ ) and $\iota^{\prime}$ accesses $X$ next (since $\iota^{\prime}$ accesses $X$ next in $Q^{\prime}$ and $Q^{\prime} / \iota=Q^{\prime \prime} / \iota$ ).

Consider a step on thread $\iota$ from some state $(H, Q)$ in our execution sequence $\bar{\alpha}$. In order for this step to affect the behavior of another thread in a later step of the sequence, this step must modify the global store. There are only three rules that directly modify the store:

EC-SYNCACQuire So $Q . c m d(\iota)$ has the form $\mathcal{C}\left[\right.$ sync $\ell$ do $\left.c_{0}\right]$ and $\ell \in H$ and $Q . l s(\iota) . w b=n i l$. But then by construction of our SC execution in Lemma 55, we know that there is some $j>k$ such that $P_{j} . c m d(\iota)$ has the form $\mathcal{C}\left[\right.$ sync $\ell$ do $\left.c_{0}\right]$ and $\ell \in G_{j}$. Further, by well-behavedness we know that $\ell \notin P_{j} . l s(\iota)$. Therefore, the step from $\left(G_{j}, P_{j}\right)$ must also use EC-SYNCACQUiRE. But then $P_{j} . l s(\iota) \cdot w b=n i l$, which contradicts the fact that a write to $X$ is in $P_{j} . l s(\iota) . w b$.

EC-HoldRelease Similar to the above case.

commit By construction of our SC execution in Lemma 55, a commit step only happens on thread $\iota$ directly after the associated write is put into $\iota$ 's write buffer. Further, there is a corresponding write to the write buffer of $\iota$ in the original TSO execution. So there is some $j \geq k$ such that $P_{j} . c m d(\iota)$ has the form $\mathcal{C}[Y:=y]$ and $\left(G_{j}, P_{j}\right)$ executes thread $\iota$, thereby adding $Y$ to $\iota$ 's write buffer. By our choice of $\left(G_{k}, P_{k}\right)$ we know that the step from $\left(G_{k}, P_{k}\right)$ adds a write to $X$ to the write buffer of $\iota$ and that this write is never committed in the rest of our execution sequence. Since the write buffer is emptied in FIFO order, this means that the write to $Y$ at $\left(G_{j}, P_{j}\right)$ is also never committed (note that it is possible that $j=k$ and hence $Y=X)$. Therefore by well-behavedness, in each $\left(G_{m}, P_{m}\right)$ for $j \leq m \leq n$ it is the case that no thread other than $\iota$ accesses $Y$ next. Therefore, the same is true for each configuration following $\left(H_{j}, Q_{j}\right)$ in our SC execution sequence $\bar{\alpha}$. Therefore, we can omit this write of $Y$ without affecting any other thread's behavior in the rest of the execution sequence.

Theorem 2. If $\left(G,\left\langle L_{\oslash}, c\right\rangle\right) \Longrightarrow^{\text {tso* }}\left(G^{\prime}, \mathrm{o}\right)$ and $\left(G,\left\langle L_{\oslash}, c\right\rangle\right)$ is race-free, then $\left(G,\left\langle L_{\oslash}, c\right\rangle\right) \Longrightarrow^{\mathrm{sc} *}\left(G^{\prime}, \mathrm{o}\right)$.

Proof. Since $\left(G,\left\langle L_{\oslash}, c\right\rangle\right)$ is race-free, by Lemma 56 each intermediate configuration in execution $\left(G,\left\langle L_{\oslash}, c\right\rangle\right) \Longrightarrow{ }^{\text {tso* }}\left(G^{\prime}, \mathcal{o}\right)$ is well behaved. By Definition $20,\left(G,\left\langle L_{\oslash}, c\right\rangle\right)$ is its own commit closure. Therefore by Lemma 55 we have $\left(G,\left\langle L_{\oslash}, c\right\rangle\right) \Longrightarrow^{\text {sc* }}\left(H^{\prime}, Q^{\prime}\right)$ where $\left(H^{\prime}, Q^{\prime}\right)$ is the commit closure of $\left(G^{\prime}, \mathrm{o}\right)$. But then by Definition 20, $Q^{\prime}=\mathrm{o}$ so also $H^{\prime}=G^{\prime}$.

\section{$5 \quad$ Typing for Write Buffers}

Write buffers are not as cumbersome as in the proofs about the previous type system, and we won't need to call them out specially in contexts. Notation $\mathcal{E}[t]$ denotes $\mathcal{E}[n i l \mid t]$. 


$$
\begin{aligned}
& p c ; w t ; \Gamma \vdash^{\mathrm{wb}} c \Rightarrow w t \\
& \frac{p c \sqcup \Gamma(Y) \sqsubseteq \Gamma(x)}{p c ; w t ; \Gamma \vdash^{\mathrm{wb}} x:=Y \Rightarrow w t} \text { wB-LoAD } \quad \frac{p c \sqcup \Gamma(y) \sqsubseteq \Gamma(X)}{p c ; w t ; \Gamma \vdash^{\mathrm{wb}} X:=y \Rightarrow w t \sqcap \Gamma(X)} \text { wB-StorE } \\
& \frac{\Gamma \vdash a: \tau \quad p c \sqcup \tau \sqsubseteq \Gamma(x)}{p c ; w t ; \Gamma \vdash^{\mathrm{wb}} x:=a \Rightarrow w t} \text { WB-EvaL } \quad \frac{p c \sqsubseteq \Gamma(\ell) \quad p c \sqsubseteq w t \quad \Gamma(\ell) ; h i g h ; \Gamma \vdash^{\mathrm{wb}} c \Rightarrow w t^{\prime}}{p c ; w t ; \Gamma \vdash^{\text {wb }} \text { sync } \ell \text { do } c \Rightarrow h i g h} \text { WB-SYNC } \\
& \frac{p c \sqsubseteq \Gamma(\ell) \quad \Gamma(\ell) \sqsubseteq w t \quad \Gamma(\ell) ; w t ; \Gamma \vdash^{\mathrm{wb}} c \Rightarrow w t^{\prime}}{p c ; w t ; \Gamma \vdash^{\mathrm{wb}} \text { holding } \ell \text { do } c \Rightarrow h i g h} \text { wB-HoLD } \quad \frac{p c \sqsubseteq w t}{p c ; w t ; \Gamma \vdash^{\mathrm{wb}} \text { fence } \Rightarrow \text { high }} \text { WB-FENCE } \\
& \frac{p c ; \text { high; } \Gamma \vdash^{\mathrm{wb}} c \Rightarrow w t^{\prime} \quad p c \sqsubseteq w t}{p c ; w t ; \Gamma \vdash^{\mathrm{wb}} \text { fork } c \Rightarrow \text { high }} \text { wB-FORK } \\
& \frac{p c ; w t ; \Gamma \vdash^{\mathrm{wb}} c_{1} \Rightarrow w t_{1} \quad p c ; w t_{1} ; \Gamma \vdash^{\mathrm{wb}} c_{2} \Rightarrow w t_{2}}{p c ; w t ; \Gamma \vdash^{\mathrm{wb}} c_{1} ; c_{2} \Rightarrow w t_{2}} \text { wB-SEQ } \\
& \frac{\Gamma \vdash b: \tau \quad p c \sqcup \tau ; w t ; \Gamma \vdash^{\mathrm{wb}} c_{1} \Rightarrow w t_{1} \quad p c \sqcup \tau ; w t ; \Gamma \vdash^{\mathrm{wb}} c_{2} \Rightarrow w t_{2}}{p c ; w t ; \Gamma \vdash^{\mathrm{wb}} \text { if } b \text { do } c_{1} \text { else } c_{2} \Rightarrow w t_{1} \sqcap w t_{2}} \text { wB-IF }
\end{aligned}
$$

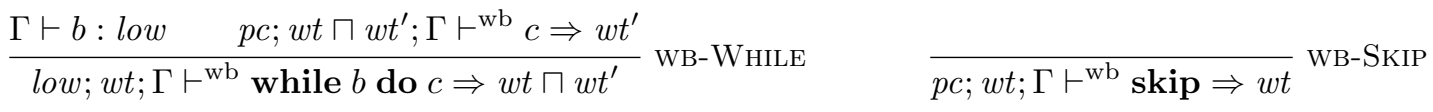

$$
\begin{aligned}
& p c ; \Gamma \vdash^{\mathrm{wb}} \lambda \\
& \overline{p c ; \Gamma \vdash^{\mathrm{wb}} \emptyset} \quad \frac{p c \sqsubseteq \Gamma(\ell) \quad p c ; \Gamma \vdash^{\mathrm{wb}} \lambda}{p c ; \Gamma \vdash^{\mathrm{wb}} \lambda \cup\{\ell\}} \\
& w t ; \Gamma \vdash^{\mathrm{wb}} W \\
& \frac{w t \sqsubseteq \Gamma(X) \quad w t ; \Gamma \vdash^{\mathrm{wb}} W}{w t ; \Gamma \vdash^{\mathrm{wb}}(X:=i):: W} \\
& p c ; w t ; \Gamma \vdash^{\mathrm{wb}} t \\
& \frac{p c ; \Gamma \vdash^{\mathrm{wb}} \lambda \quad w t ; \Gamma \vdash^{\mathrm{wb}} W \quad p c ; w t ; \Gamma \vdash^{\mathrm{wb}} c \Rightarrow w t^{\prime}}{p c ; w t ; \Gamma \vdash^{\mathrm{wb}}\langle(M, \lambda, W), c\rangle_{\iota}} \\
& \overline{p c} ; \overline{w t} ; \Gamma \vdash^{\mathrm{wb}} P \\
& \frac{p c ; w t ; \Gamma \vdash^{\mathrm{wb}} t \quad \overline{p c} ; \overline{w t} ; \Gamma \vdash^{\mathrm{wb}} P}{p c, \overline{p c} ; w t, \overline{w t} ; \Gamma \vdash^{\mathrm{wb}} t \| P}
\end{aligned}
$$

Lemma 57 (WB Admissibility of subtyping). Suppose $p c_{1} \sqsubseteq p c_{2}$ and $w t_{1} \sqsubseteq w t_{2}$. Then the following hold. 
(i) $p c_{2} ; \Gamma \vdash^{\mathrm{wb}} \lambda$ implies $p c_{1} ; \Gamma \vdash^{\mathrm{wb}} \lambda$.

(ii) $w t_{2} ; \Gamma \vdash^{\mathrm{wb}} W$ implies $w t_{1} ; \Gamma \vdash^{\mathrm{wb}} W$.

(iii) $p c_{2} ; w t_{1} ; \Gamma \vdash^{\mathrm{wb}} c \Rightarrow$ t implies $p c_{1} ; w t_{2} ; \Gamma \vdash^{\mathrm{wb}} c \Rightarrow v t$ for some vt where $u t \sqsubseteq v t$.

(iv) $p c_{2} ; w t ; \Gamma \vdash^{\mathrm{wb}} t$ implies $p c_{1} ; w t ; \Gamma \vdash^{\mathrm{wb}} t$.

Proof. Statements (i)-(iii) follow from easy inductions on the typing derivations. The only mildly interesting cases are for WB-SEQ, WB-IF, and WB-WHILE, in which we need to reason with the induction hypothesis's $u t \sqsubseteq v t$ constraint. Statement (iv) follows from (i)-(iii).

Lemma 58. Suppose $p c ; w t ; \Gamma \vdash^{\mathrm{wb}} c \Rightarrow v t$. Then $p c \sqcap w t \sqsubseteq v t$.

Proof. by an easy induction.

Lemma 59. From $p c \sqsubseteq \Gamma(\ell)$ and $p c ; \Gamma \vdash^{\text {wb }} \lambda$, it follows that $p c ; \Gamma \vdash^{\text {wb }} \lambda \cup\{\ell\}$.

Proof. Immediate.

Lemma 60. Suppose $p c ; \Gamma \vdash^{\mathrm{wb}} \lambda$. It follows that $p c ; \Gamma \vdash^{\mathrm{wb}} \lambda \backslash\{\ell\}$.

Proof. by trivial induction.

Lemma 61 (WB buffer append typing). If $w t ; \Gamma \vdash^{\text {wb }} W$ then $w t \sqcap \Gamma(X) ; \Gamma \vdash^{\text {wb }} W+(X:=i)$.

Proof. by induction.

Lemma 62 (WB Commit Step Preservation). Suppose $p c ; w t ; \vdash^{\text {wb }} t$ and $(G, t) \longrightarrow{ }^{\text {commit }}\left(G^{\prime}, P^{\prime}\right)$. Then $\overline{p c} ; \overline{w t} ; \Gamma \vdash^{\mathrm{wb}} P^{\prime}$ where $p c \sqsubseteq \overline{p c}$ and $w t \sqsubseteq \overline{w t}$.

Proof. Let $\langle L, c\rangle=t$. Then $P^{\prime}=\left\langle L_{0}, c\right\rangle \|$ o where $L=(X:=i)+L_{0}$ for some $X, i$, and $L_{0}$. We must show that $w t ; \Gamma \vdash^{\mathrm{wb}} L_{0} . w b$, which follows from inverting the typing derivation.

Lemma 63 (WB Eval Step Preservation). Suppose $p c ; w t ; \Gamma \vdash^{\text {wb }} t$ and $(G, t) \longrightarrow^{\text {eval }}\left(G^{\prime}, P^{\prime}\right)$. Then $\overline{p c} ; \overline{w t} ; \Gamma \vdash^{\text {wb }} P^{\prime}$ where $p c \sqsubseteq \overline{p c}$ and $w t \sqcap p c \sqsubseteq \overline{w t}$.

Proof. Let $\langle L, c\rangle=t$ and observe that $p c ; w t ; \Gamma \vdash^{\mathrm{wb}} c \Rightarrow u t$. Strengthen the induction hypothesis as follows: If $P^{\prime}=t^{\prime} \| P_{0}^{\prime}$, it is the case that $v t ; \Gamma \vdash^{\mathrm{wb}} t^{\prime} . w b$ and $p c ; v t ; \Gamma \vdash^{\mathrm{wb}} t^{\prime} . c m d \Rightarrow u t^{\prime}$ where $w t \sqcap p c \sqsubseteq v t$ and $u t \sqsubseteq u t^{\prime}$. Proceed by induction on the $\longrightarrow^{\text {eval }}$ step derivation.

EC-Store: Here $c=(X:=y)$ and $P^{\prime}=\langle L+(X:=i)$, skip $\rangle \| \mathcal{o}$ for some $i$. It suffices to find $v t$ such that (i) $w t \sqcap p c \sqsubseteq v t$, (ii) $v t ; \Gamma \vdash^{\mathrm{wb}} L . w b+(X:=i)$, and (iii) $p c ; v t ; \Gamma \vdash^{\mathrm{wb}}$ skip $\Rightarrow u t$. Taking $v t=u t$ latter is immediate. Inverting the typing relation shows $p c \sqsubseteq p c \sqcup \Gamma(y) \sqsubseteq \Gamma(X)$ and $v t=u t=w t \sqcap \Gamma(X) \sqsupseteq$ $w t \sqcap p c$, satisfying (i). Finally (ii) follows from Lemma 61.

EC-LOAD: Here $c=(x:=Y)$ and $P^{\prime}=\langle L[X \mapsto i]$, skip $\rangle \|$ o. Inverting the typing relation shows $u t=w t$. Taking $v t=w t \sqsupseteq w t \sqcup p c$, it suffices to show $v t ; \Gamma \vdash^{\text {wb }} L$. wb, which follows from inverting the typing relation and $p c ; v t ; \Gamma \vdash^{\mathrm{wb}} L . w b \Rightarrow u t$, which is immediate.

EC-EvalExP: Similar to the EC-LOAD case.

EC-SYNCACQUiRe: Here $c=\operatorname{sync} \ell$ do $c_{0}$ and $P^{\prime}=\left\langle L \cup\{\ell\}\right.$, holding $\ell$ do $\left.\left.c_{0}\right)\right\rangle \| \mathcal{o}$ and L.wb $=$ nil. Inverting the typing relation gives $u t=h i g h$ and $p c \sqsubseteq \Gamma(\ell)$ and $\Gamma(\ell) ; h i g h ; \Gamma \vdash^{\mathrm{wb}} c_{0} \Rightarrow u t_{0}$. Because $L$ 's lock set changed, we must show that $p c ; \Gamma \vdash^{\text {wb }} L$.locks $\cup\{\ell\}$, which is immediate from Lemma 59. Let $v t=h i g h$. Conclude by constructing derivations of $h i g h ; \Gamma \vdash^{\mathrm{wb}} L . w b$ and $p c ; h i g h ; \Gamma \vdash^{\mathrm{wb}}$ holding $\ell$ do $c_{0} \Rightarrow$ high.

EC-SyncReenter: Here $c=$ sync $\ell$ do $c_{0}$ and $P^{\prime}=\left\langle L\right.$, fence; $\left(c_{0}\right.$; fence $\left.)\right\rangle \| \mathcal{o}$. Inverting the typing relation shows $u t=h i g h$ and $p c \sqsubseteq w t$. Inversion also gives $\Gamma(\ell) ; h i g h ; \Gamma \vdash^{\mathrm{wb}} c_{0} \Rightarrow w t_{0}$ and $p c \sqsubseteq \Gamma(\ell)$ so 
that Lemma 57 yields a derivation showing $p c ;$ high; $\Gamma \vdash^{\text {wb }} c_{0} \Rightarrow w t_{0}^{\prime}$ with $w t_{0} \sqsubseteq w t_{0}^{\prime}$. By Lemma 58 , $p c \sqsubseteq w t_{0}^{\prime}$. Taking $v t=w t$, it remains to construct the following derivation:

$$
\frac{\frac{p c \sqsubseteq w t}{p c ; w t ; \Gamma \vdash^{\mathrm{wb}} \text { fence } \Rightarrow h i g h} \quad \frac{\frac{\vdots}{p c ; h i g h ; \Gamma \vdash^{\mathrm{wb}} c_{0} \Rightarrow w t_{0}^{\prime}}}{p c ; h i g h ; \Gamma \vdash^{\mathrm{wb}} c_{0} ; \text { fence } \Rightarrow h i g h} \frac{p c \sqsubseteq w t_{0}^{\prime}}{p c ; w t_{0}^{\prime} ; \Gamma \vdash^{\mathrm{wb}} \text { fence } \Rightarrow h i g h}}{p c ; w t ; \Gamma \vdash^{\mathrm{wb}} \text { fence } ;\left(c_{0} ; \text { fence }\right) \Rightarrow h i g h}
$$

EC-HoldStep: Here $c=$ holding $\ell$ do $c_{0}$ and $P^{\prime}=\left\langle L^{\prime}\right.$, holding $\ell$ do $\left.c_{0}^{\prime}\right\rangle \| P_{0}^{\prime}$ where $\left(G,\left\langle L, c_{0}\right\rangle\right) \longrightarrow^{\text {eval }}$ $\left(G^{\prime},\left\langle L^{\prime}, c_{0}^{\prime}\right\rangle \| P_{0}^{\prime}\right)$. Inverting the typing derivation gives $\Gamma(\ell) ; w t ; \Gamma \vdash^{\mathrm{wb}} c_{0} \Rightarrow w t_{0}$ and $p c \sqsubseteq \Gamma(\ell)$. Applying the induction hypothesis yields $\overline{p c} ; \overline{w t} ; \Gamma \vdash^{\text {wb }} P^{\prime}$ where $p c \sqsubseteq \Gamma(\ell) \sqsubseteq \overline{p c}$ and $p c \sqcap w t \sqsubseteq$ $\Gamma(\ell) \sqcap w t \sqsubseteq \overline{w t}$. Thus $P_{0}^{\prime}$ is typed appropriately, and it remains to shows that $\left\langle L^{\prime}\right.$, holding $\left.\ell \mathbf{d o} c_{0}^{\prime}\right\rangle$ satisfies all typing requirements. Also by the induction hypothesis $\Gamma(\ell) ; v t ; \Gamma \vdash^{\text {wb }} c_{0}^{\prime} \Rightarrow w t_{0}^{\prime}$ and $w t \sqcap \Gamma(\ell) \sqsubseteq v t$. It remains to show that $p c ; v t ; \Gamma \vdash^{\text {wb }}$ holding $\ell$ do $c_{0}^{\prime} \Rightarrow h i g h$, which follows from rule WB-HoLD and using fact $\overline{p c} ; \overline{w t} ; \Gamma \vdash^{\mathrm{wb}} P^{\prime}$ to establish $p c ; \Gamma \vdash^{\mathrm{wb}} L^{\prime}$.locks.

EC-HoldRelease: Here $c=$ holding $\ell$ do skip and $P^{\prime}=\langle L \backslash\{\ell\}$, skip $\rangle \|$ o with $L$.wb $=$ nil. Inverting the typing relation shows $u t=h i g h$. Let $v t=h i g h$. Proceed as in the EC-LOAD step, noting that Lemma 60 gives $p c ; \Gamma \vdash^{\text {wb }}$ L.locks $\backslash\{\ell\}$ and high; $\Gamma \vdash^{\text {wb }}$ L.wb is immediate.

EC-Fence: Similar to, but simpler than EC-HoldRelease.

EC-ForK: Here $c=$ fork $c_{0}$ and $P^{\prime}=\langle L$, skip $\rangle\left\|\left\langle L_{\oslash}, c_{0}\right\rangle\right\| \mathcal{o}$ and $L . w b=$ nil.

First we show that $p c ;$ high; $\Gamma \vdash^{\mathrm{wb}}\left\langle L_{\oslash}, c_{0}\right\rangle \| \mathrm{o}$. Inverting the typing relation gives $p c ;$ high; $\Gamma \vdash^{\mathrm{wb}} c_{0} \Rightarrow$ $w t_{0}$. Finish building easy derivations of $p c ; \Gamma \vdash^{\mathrm{wb}} L_{\oslash}$.locks and high; $\Gamma \vdash^{\mathrm{wb}} L_{\oslash}$.wb.

Second we let $v t=u t=h i g h$ and show $p c ; h i g h ; \Gamma \vdash^{\text {wb }}$ skip $\Rightarrow h i g h$, and high; $\Gamma \vdash^{\text {wb }}$ L.wb both of which are immediate.

EC-SeqStruct: Here $c=c_{1} ; c_{2}$ and $P^{\prime}=\left\langle L^{\prime}, c_{1}^{\prime}\right\rangle \| P_{0}^{\prime}$ where $\left(G,\left\langle L, c_{1}\right\rangle\right) \longrightarrow^{\text {eval }}\left(G^{\prime},\left\langle L^{\prime}, c_{1}^{\prime}\right\rangle \| P_{0}^{\prime}\right)$. Inverting the typing derivation gives $p c ; w t ; \Gamma \vdash^{\mathrm{wb}} c_{1} \Rightarrow w t_{1}$ and $p c ; w t_{1} ; \Gamma \vdash^{\mathrm{wb}} c_{2} \Rightarrow u t$.

The induction hypothesis shows $\overline{p c} ; \overline{w t} ; \Gamma \vdash^{\text {wb }} P_{0}^{\prime}$ where $p c \sqsubseteq \overline{p c}$ and $w t \sqcap p c \sqsubseteq \overline{w t}$.

Conclude by building a derivation of $p c ; v t ; \Gamma \vdash^{\mathrm{wb}} c_{1}^{\prime} ; c_{2} \Rightarrow u t^{\prime}$ using the induction hypothesis to find and appropriate $v t$ where $p c ; v t ; \Gamma \vdash^{\text {wb }} c_{1}^{\prime} \Rightarrow w t_{1}^{\prime}$ and $w t_{1} \sqsubseteq w t_{1}^{\prime}$ and computing $u t^{\prime}$ as follows. Use Lemma 57 to find $p c ; w t_{1}^{\prime} ; \Gamma \vdash^{\mathrm{wb}} c_{2} \Rightarrow u t^{\prime}$ where $u t \sqsubseteq u t^{\prime}$, then build a derivation $p c ; v t ; \Gamma \vdash^{\mathrm{wb}} c_{1}^{\prime} ; c_{2} \Rightarrow$ ut with rule WB-SEQ.

EC-SEQSkIP: Here $c=\operatorname{skip} ; c_{2}$ and $P^{\prime}=\left\langle L, c_{2}\right\rangle \| \mathcal{o}$. Trivial by inverting the typing derivation.

EC-IfTrue, eC-IfFalse, eC-WhileTrue, eC-WhileFalse: Immediate, using Lemma 57 as needed.

EC-REAP: Immediate.

Lemma 64 (WB Preservation). Suppose $\overline{p c} ; \overline{w t} ; \Gamma \vdash^{\text {wb }} P$ and $(G, P) \Longrightarrow^{\text {tso* }}\left(G^{\prime}, P^{\prime}\right)$. Then there exist $\overline{p c}^{\prime}$ and $\overline{w t}^{\prime}$ such that $\overline{p c}^{\prime} ; \overline{w t}^{\prime} ; \Gamma \vdash^{\text {wb }} P^{\prime}$.

Proof. By induction on the length of the $\Longrightarrow^{\text {tso* }}$ derivation, using Lemmas 62 or 63.

Definition $21\left(\sim_{\Gamma}^{\mathrm{wb}}\right)$.

1. $\lambda_{1} \sim_{\Gamma}^{\mathrm{wb}} \lambda_{2}$ when it is the case that $\Gamma(\ell)=$ low implies that $\ell \in \lambda_{1}$ iff $\ell \in \lambda_{2}$.

2. $L_{1} \sim_{\Gamma}^{\mathrm{wb}} L_{2}$ iff each of the following holds:

(a) $L_{1} . w b \sim_{\Gamma} L_{2} . w b$

(b) $L_{1}$. mem $\sim_{\Gamma} L_{2}$. mem

(c) $L_{1}$.locks $\sim_{\Gamma}^{\mathrm{wb}} L_{2}$.locks

3. $t_{1} \sim \sim_{\Gamma}^{\mathrm{wb}} t_{2}$ is defined by the following introduction rules. 
(a) $\left\langle L_{1}, c\right\rangle_{\iota_{1}} \sim_{\Gamma}^{\mathrm{wb}}\left\langle L_{2}, c\right\rangle_{\iota_{2}}$ when $L_{1} \sim_{\Gamma}^{\mathrm{wb}} L_{2}$

(b) $\mathcal{E}\left[\left\langle L_{1}, c_{1}\right\rangle_{\iota_{1}}\right] \sim_{\Gamma}^{\mathrm{wb}} \mathcal{E}\left[\left\langle L_{2}, c_{2}\right\rangle_{\iota_{2}}\right]$ when $L_{1} \sim_{\Gamma}^{\mathrm{wb}} L_{2}$ and both high; wt; $\Gamma \vdash^{\mathrm{wb}}\left\langle L_{1}, c_{1}\right\rangle_{\iota_{1}}$ and high; wt; $\Gamma \vdash^{\mathrm{wb}}$ $\left\langle L_{2}, c_{2}\right\rangle_{\iota_{2}}$ for some wt.

4. $G_{1} \sim_{\Gamma}^{\mathrm{wb}} G_{2}$ iff $G_{1}$. mem $\sim_{\Gamma} G_{2}$. mem and $G_{1}$.locks $\sim_{\Gamma}^{\mathrm{wb}} G_{2}$.locks.

5. $P_{1} \sim{ }_{\Gamma}^{\mathrm{wb}} P_{2}$ is defined by the least fixed point of the following implications.

(a) $\mathcal{O} \sim \sim_{\Gamma}^{\mathrm{wb}} \mathcal{O}$, always

(b) $t \| P_{1} \sim_{\Gamma}^{\mathrm{wb}} P_{2}$ when high; high; $\Gamma \vdash^{\mathrm{wb}} t$ and $P_{1} \sim_{\Gamma}^{\mathrm{wb}} P_{2}$

(c) $P_{1} \sim{ }_{\Gamma}^{\mathrm{wb}} t \| P_{2}$ when high; high; $\Gamma \vdash^{\mathrm{wb}} t$ and $P_{1} \sim_{\Gamma}^{\mathrm{wb}} P_{2}$

(d) $t_{1}\left\|P_{1} \sim_{\Gamma}^{\mathrm{wb}} t_{2}\right\| P_{2}$ when $t_{1} \sim_{\Gamma}^{\mathrm{wb}} t_{2}$ and $P_{1} \sim_{\Gamma}^{\mathrm{wb}} P_{2}$

6. $\left(G_{1}, P_{1}\right) \sim_{\Gamma}^{\mathrm{wb}}\left(G_{2}, P_{2}\right)$ when $G_{1} \sim_{\Gamma}^{\mathrm{wb}} G_{2}$ and $P_{1} \sim_{\Gamma}^{\mathrm{wb}} P_{2}$.

Lemma 65. Each $\sim_{\Gamma}^{\mathrm{wb}}$ relation is an equivalence relation.

Proof. by inspection.

Lemma 66. If $\left(G_{1}, P_{1}\right) \sim_{\Gamma}^{\mathrm{wb}}\left(G_{2}, P_{22}\right)$ and $P_{21} \sim_{\Gamma}^{\mathrm{wb}} \mathcal{o}$ then $\left(G_{1}, P_{1}\right) \sim_{\Gamma}^{\mathrm{wb}}\left(G_{2}, P_{22} \| P_{21}\right)$

Lemma 67. Consider some $G_{1}, G_{2}$, and $\lambda$. $G_{1} \sim_{\Gamma}^{\mathrm{wb}} G_{2}$ iff $G_{1} \cup \lambda \sim_{\Gamma}^{\mathrm{wb}} G_{2} \cup \lambda$.

Lemma 68. Consider some $G_{1}, G_{2}$, and $\lambda . G_{1} \sim_{\Gamma}^{\mathrm{wb}} G_{2}$ iff $G_{1} \backslash \lambda \sim_{\Gamma}^{\mathrm{wb}} G_{2} \backslash \lambda$.

Lemma 69. Consider some $L_{1}, L_{2}$, and $\lambda . L_{1} \sim_{\Gamma}^{\mathrm{wb}} L_{2}$ iff $L_{1} \cup \lambda \sim_{\Gamma}^{\mathrm{wb}} L_{2} \cup \lambda$.

Lemma 70. Suppose $L_{1} \sim_{\Gamma}^{\mathrm{wb}} L_{2}$. Then $L_{1}[x \mapsto i] \sim_{\Gamma}^{\mathrm{wb}} L_{2}[x \mapsto i]$.

Lemma 71. Suppose $L_{1} \sim_{\Gamma}^{\mathrm{wb}} L_{2}$ and $\Gamma(x)=$ high. Then $L_{1}\left[x \mapsto i_{1}\right] \sim_{\Gamma}^{\mathrm{wb}} L_{2}\left[x \mapsto i_{2}\right]$.

Lemma 72. Suppose $L_{1} \sim_{\Gamma}^{\mathrm{wb}} L_{2}$. Then $L_{1}+(X:=i) \sim_{\Gamma}^{\mathrm{wb}} L_{2}+(X:=i)$.

Lemma 73. Suppose $L_{1} \sim_{\Gamma}^{\mathrm{wb}} L_{2}$ and $\Gamma(X)=$ high. Then $L_{1}+(X:=i) \sim_{\Gamma}^{\mathrm{wb}} L_{2}+(X:=j)$.

Lemma 74. Suppose $L_{1} \sim_{\Gamma}^{\mathrm{wb}} L_{2}$. Then $w t ; \Gamma \vdash^{\mathrm{wb}} L_{1}$.wb implies $w t ; \Gamma \vdash^{\mathrm{wb}} L_{2}$.wb, and $p c ; \Gamma \vdash^{\mathrm{wb}} L_{1}$.locks implies $p c ; \Gamma \vdash^{\text {wb }} L_{2}$.locks.

Proof. by trivial inductions.

Lemma 75. Suppose $t_{1} \sim_{\Gamma}^{\mathrm{wb}} t_{2}$. Then $\mathcal{E}\left[t_{1}\right] \sim_{\Gamma}^{\mathrm{wb}} \mathcal{E}\left[t_{2}\right]$.

Lemma 76. If $P_{11}\left\|t_{1}\right\| P_{12} \sim_{\Gamma}^{\mathrm{wb}} P_{2}$ then $P_{2}=P_{21}\left\|P_{2}^{*}\right\| P_{22}$ where the following hold:

$$
\begin{array}{rll}
P_{21} & \sim{ }_{\Gamma}^{\text {wb }} & P_{11} \\
P_{2}^{*} & \sim \underset{\Gamma}{\text { wb }} t_{1} \\
P_{22} & \sim{ }_{\Gamma}^{\text {wb }} & P_{12} \\
P_{2}^{*} & \in\left\{\mathfrak{o}, t_{2} \| \text { o }\right\} \text { for some } t_{2}
\end{array}
$$

Proof. By any easy induction on the sum of the lengths of $P_{1}$ and $P_{2}$.

Lemma 77. If $p c ; w t ; \Gamma \vdash^{\mathrm{wb}} \mathcal{E}[t]$ then $p c ; w t ; \Gamma \vdash^{\mathrm{wb}} t$.

Proof. Let $\mathcal{E}=(\lambda, \mathcal{C})$. First use induction on the size of $\lambda$ to show $p c ; w t ; \Gamma \vdash^{\text {wb }} \mathcal{E}_{0}[t]$ where $\mathcal{E}_{0}=(\emptyset, \mathcal{C})$. Conclude by an easy structural induction on $\mathcal{C}$, using Lemma 57.

Definition $22\left(\left.\cdot\right|_{\Gamma, \tau}\right)$.

$$
\begin{aligned}
\left.\lambda\right|_{\Gamma, \tau} & =\{\ell \mid \ell \in \lambda \text { and } \Gamma(\ell)=\tau\} \\
\left.L\right|_{\Gamma, \tau} & =L .\left.l o c k s\right|_{\Gamma, \tau} \\
\left.\langle L, c\rangle\right|_{\Gamma, \tau} & =\left.L\right|_{\Gamma, \tau} \\
\left.\mathcal{o}\right|_{\Gamma, \tau} & =\emptyset \\
\left.(t \| P)\right|_{\Gamma, \tau} & =\left.\left.t\right|_{\Gamma, \tau} \cup P\right|_{\Gamma, \tau} \\
\left.G\right|_{\Gamma, \tau} & =\text { G.locks }\left.\right|_{\Gamma, \tau}
\end{aligned}
$$


Lemma 78. If $\left.\lambda \subseteq \mathbf{L o c k}\right|_{\Gamma, \text { high }}$ and high; wt; $\Gamma \vdash^{\mathrm{wb}} t$ then high; wt; $\Gamma \vdash^{\mathrm{wb}} t \cup \lambda$.

Proof. Immediate

Lemma 79. Suppose that high; wt; $\Gamma \vdash^{\mathrm{wb}}$ t. Then t.cmd.locks $\left.\subseteq \mathbf{L o c k}\right|_{\Gamma, \text { high }}$.

Proof. by trivial induction on the typing derivation.

Lemma 80. Suppose $L_{1} \sim_{\Gamma}^{\mathrm{wb}} L_{2}$ and high; wt; $\Gamma \vdash^{\mathrm{wb}} c_{1} \Rightarrow u t_{1}$ and high; wt; $\Gamma \vdash^{\mathrm{wb}} c_{2} \Rightarrow u t_{2}$. Suppose also that $w t ; \Gamma \vdash^{\mathrm{wb}} L_{1}$.wb. Then $\left\langle L_{1}, c_{1}\right\rangle \sim_{\Gamma}^{\mathrm{wb}}\left\langle L_{2}, c_{2}\right\rangle$.

Proof. By the definition of $\sim^{\text {wb }}$ we want to find $\mathcal{E}=(\lambda, \mathcal{C}), L_{10}$, and $L_{20}$, such that the following hold.

$$
\left\langle L_{1}, c_{1}\right\rangle=\mathcal{E}\left[\left\langle L_{10}, c_{1}\right\rangle\right] \quad\left\langle L_{2}, c_{2}\right\rangle=\mathcal{E}\left[\left\langle L_{20}, c_{2}\right\rangle\right] \quad h i g h ; w t ; \Gamma \vdash^{\mathrm{wb}}\left\langle L_{10}, c_{1}\right\rangle \quad h i g h ; w t ; \Gamma \vdash^{\mathrm{wb}}\left\langle L_{20}, c_{2}\right\rangle
$$

Let $\mathcal{C}=[\cdot]$ and $\lambda=\left.L_{1}\right|_{\Gamma, \text { low }}$. Let also $L_{10}=L_{1} \backslash \lambda$ and $L_{20}=L_{2} \backslash \lambda$. Clearly $\left\langle L_{1}, c_{1}\right\rangle=\mathcal{E}\left[\left\langle L_{10}, c_{1}\right\rangle\right]$. Because $L_{1} \sim_{\Gamma}^{\text {wb }} L_{2}$ it's also true that $\lambda=\left.L_{2}\right|_{\Gamma, \text { low }}$, so $\left\langle L_{2}, c_{2}\right\rangle=\mathcal{E}\left[\left\langle L_{20}, c_{2}\right\rangle\right]$. We demonstrate high; wt; $\Gamma \vdash^{\text {wb }}$ $\left\langle L_{10}, c_{1}\right\rangle$ by using the definition of $L_{10}$ and the premises to show that high; $\Gamma \vdash^{\text {wb }} L_{10}$.locks and $w t ; \Gamma \vdash^{\text {wb }}$ $L_{10} \cdot w b$. It remains to show high; wt; $\Gamma \vdash^{\text {wb }}\left\langle L_{20}, c_{2}\right\rangle$, which works as above, using Lemma 74 to help establish $w t ; \Gamma \vdash^{\text {wb }} L_{20} . w b$.

Lemma 81 (WB Eval step local confinement). Suppose $t . c m d \neq$ skip and both high; wt; $\Gamma \vdash^{\text {wb }} t$ and $(G, \mathcal{E}[t]) \longrightarrow^{\text {eval }}\left(G^{\prime}, P^{\prime}\right)$. Then $(G, \mathcal{E}[t]) \sim_{\Gamma}^{\text {wb }}\left(G^{\prime}, P^{\prime}\right)$, and $P^{\prime}=t^{\prime} \| P_{0}^{\prime}$ where $\mathcal{E}[t] \sim_{\Gamma}^{\text {wb }} t^{\prime}$.

Proof. Let $(\lambda, \mathcal{C})=\mathcal{E}$, define $t_{0}=\left.t \cup \lambda\right|_{\Gamma \text {,high }}$, and observe that by Lemma 79 , $t_{0}$.ls.locks $\supseteq \lambda \cap t_{0}$.cmd.locks. Apply Lemma 33 to reduction $(G, \mathcal{E}[t])=\left(G, \mathcal{E}\left[n i l \mid t_{0}\right]\right) \longrightarrow^{\text {eval }}\left(G^{\prime}, P^{\prime}\right)$ to find $P^{\prime}=\mathcal{E}\left[t^{\prime}\right] \| P_{0}^{\prime}$ and $\left(G, t_{0}\right) \longrightarrow^{\text {eval }}\left(G^{\prime}, t^{\prime} \| P_{0}^{\prime}\right)$.

A low write, acquiring a low lock, or releasing a low lock would contradict t's high type. Therefore $G \sim \sim_{\Gamma}^{\mathrm{wb}} G^{\prime}$.

We show that $P_{0}^{\prime} \sim_{\Gamma}^{\text {wb }}$ o. If the step was not by EC-Fork this is trivial. Otherwise $P_{0}^{\prime}=\left\langle L_{\oslash}, c_{0}\right\rangle \|$ o and it remains to show that high; high; $\Gamma \vdash^{\mathrm{wb}} c_{0} \Rightarrow u t$ for some ut. This follows from inverting $t$ 's typing derivation.

Finally we show that $\mathcal{E}[t]=\mathcal{E}\left[t_{0}\right] \sim_{\Gamma}^{\text {wb }} \mathcal{E}\left[t^{\prime}\right]$. Again, acquiring or releasing a low lock, or putting a low write in the write buffer, would contradict $t_{0}$ 's high type. Therefore $t_{0} . l s \sim_{\Gamma}^{\mathrm{wb}} t^{\prime}$.ls. From the type of $t$ is follows that $h i g h ; w t ; \Gamma \vdash^{\mathrm{wb}} t_{0}$, and preservation (Lemma 63 ) shows $h i g h ; v t ; \Gamma \vdash^{\mathrm{wb}} t^{\prime}$ for some $v t$ where $w t \sqsubseteq v t$. To conclude we must show that $t_{0}$ and $t^{\prime}$ may be typed with the same write typing. If $t_{0}$.wb contains a low write then so does $t^{\prime} . w b$ and because $w t=v t=l o w$ we're done. If $t_{0} . w b$ does not contain a low write then inverting the typing derivation and using Lemma 57 shows $h i g h ; v t ; \Gamma \vdash^{\text {wb }} t_{0}$, allowing us to conclude.

Lemma 82 (WB Commit step confinement). Suppose $p c ;$ high $; \Gamma \vdash^{\text {wb }} t$ and $(G, t) \longrightarrow^{\text {commit }}\left(G^{\prime}, P^{\prime}\right)$. Then $(G, t) \sim_{\Gamma}^{\mathrm{wb}}\left(G^{\prime}, P^{\prime}\right)$.

Proof. Suppose a high value is committed; then the conclusion is immediate. Suppose instead a low value is committed, this contradicts they typing of $t$.

Lemma 83 (WB Global confinement). Suppose high; high; $\Gamma \vdash^{\text {wb }} t$ and $(G, t) \longrightarrow^{o p}\left(G^{\prime}, P^{\prime}\right)$. Then $(G, t \| \mathcal{o}) \sim_{\Gamma}^{\mathrm{wb}}\left(G^{\prime}, P^{\prime}\right)$.

Proof. Suppose $o p=$ commit. Conclude via Lemma 82. Instead suppose $o p=e v a l$ and the step is not by ECREAP. Inverting the step relation shows $t$ can be rewritten in form $\mathcal{E}[\langle L, c\rangle]$ where $c \neq$ skip and we conclude via Lemma 81. Finally suppose the step is by EC-REAP and conclude by observing $P^{\prime}=\mathfrak{o} \sim_{\Gamma}^{\mathrm{wb}} t \| \mathrm{o}$.

Lemma 84 (WB Expression Confinement). Suppose $L_{1} \sim_{\Gamma}^{\mathrm{wb}} L_{2}$. Then low; $\Gamma \vdash^{\text {tso }}$ a implies $i_{1}=i_{2}$ when $L_{1}[a] \Downarrow i_{1}$ and $L_{2}[a] \Downarrow i_{2}$. Likewise, low; $\Gamma \vdash^{\text {tso }} b$ implies $\beta_{1}=\beta_{2}$ when $L_{1}[b] \Downarrow \beta_{1}$ and $L_{2}[b] \Downarrow \beta_{2}$.

Proof. by induction

Lemma 85 (Strong inversion for $\sim^{\mathrm{wb}}$ ). Suppose $t_{1} \sim_{\Gamma}^{\mathrm{wb}} t_{2}$. Then at least one the following conditions holds. Either,

(i) $t_{1}=\left\langle L_{1}, c\right\rangle$ and $t_{2}=\left\langle L_{2}, c\right\rangle$ where $L_{1} \sim_{\Gamma}^{\mathrm{wb}} L_{2}$, or 
(ii) $t_{1}=\mathcal{E}\left[\left\langle L_{1}, c_{1}\right\rangle\right]$ where $c_{1} \neq$ skip and high; wt; $\Gamma \vdash^{\text {wb }}\left\langle L_{1}, c_{1}\right\rangle$ for some wt, or

(iii) $t_{1}=\mathcal{E}\left[\left\langle L_{1}\right.\right.$, skip $\left.\rangle\right]$ and $t_{2}=\mathcal{E}\left[\left\langle L_{2}, c_{2}\right\rangle\right]$, where the following hold for some wt:

$$
c_{2} \neq \text { skip, } \quad L_{1} \sim_{\Gamma}^{\mathrm{wb}} L_{2}, \quad \text { canEval } t_{1} \text { implies active } \mathcal{E}, \quad \text { high; wt; } \Gamma \vdash^{\mathrm{wb}}\left\langle L_{1}, c_{1}\right\rangle \text {, and }
$$

$$
\text { high; wt } ; \Gamma \vdash^{\mathrm{wb}}\left\langle L_{2}, c_{2}\right\rangle \text {. }
$$

Proof. Suppose that $t_{1}$ and $t_{2}$ are related by Definition 21, case 3a. Conclude as (i) is satisfied.

Suppose instead that $t_{1}$ and $t_{2}$ are related by case $3 \mathrm{~b}$, so Then $t_{1}=\mathcal{E}\left[\left\langle L_{1}, c_{1}\right\rangle\right]$ and $t_{2}=\mathcal{E}\left[\left\langle L_{2}, c_{2}\right\rangle\right]$ and both $h i g h ; w t ; \Gamma \vdash^{\text {wb }}\left\langle L_{1}, c_{1}\right\rangle$ and $h i g h ; w t ; \vdash^{\text {wb }}\left\langle L_{2}, c_{2}\right\rangle$. Additionally $L_{1} \sim_{\Gamma}^{\text {wb }} L_{2}$. If $c_{1} \neq$ skip then condition (ii) is satisfied. Suppose instead that $c_{1}=$ skip. If $c_{2}=$ skip then using Lemma 69 we see that condition (i) is satisfied. Now suppose $c_{2} \neq$ skip. If it's not the case that canEval $t_{1}$ then (iii) is satisfied and we conclude. Otherwise use Lemma 2 to find $\mathcal{E}_{0}$ where active $\mathcal{E}_{0}$ and $\mathcal{E}_{0}\left[t_{i}\right]=\mathcal{E}\left[t_{i}\right]$. Conclude as condition (iii) is satisfied.

Quiet traces relate consecutive trace components with by $\sim^{\text {wb }}$.

Definition 23 (Quiet traces). Call trace $\mathcal{T}=\left(G_{1}, P_{1}\right),\left(G_{2}, P_{2}\right), \ldots,\left(G_{n}, P_{n}\right)$ quiet in context $\Gamma$, written quiet $_{\Gamma} \mathcal{T}$, when for each pair of consecutive configurations, $\left(G_{i}, P_{i}\left\|t_{i}\right\| R_{i}\right)$ and $\left(G_{i+1}, P_{i}\left\|Q_{i+1}\right\| R_{i}\right)$ where $\left(G_{i}, t_{i}\right) \longrightarrow^{o p}\left(G_{i+1}, Q_{i+1}\right)$, one or more equivalences hold. First, $\left(G_{i}, t_{i} \| \mathrm{o}\right) \sim_{\Gamma}^{\mathrm{wb}}\left(G_{i+1}, Q_{i+1}\right)$. Second, if $Q_{i+1}=t_{i+1} \| Q_{i+1}^{0}$ then $t_{i+1} \sim_{\Gamma}^{\text {wb }} t_{i}$ and $Q_{i+1}^{0} \sim_{\Gamma}^{\text {wb }} \mathcal{o}$.

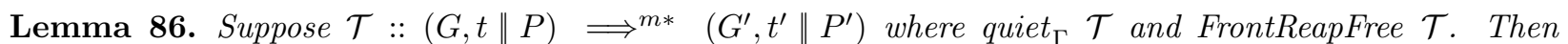
$t \sim_{\Gamma}^{\mathrm{wb}} t^{\prime}$ and $P \sim_{\Gamma}^{\mathrm{wb}} P^{\prime}$ and $G \sim_{\Gamma}^{\mathrm{wb}} G^{\prime}$.

Proof. Proof by an easy induction on the length of $\mathcal{T}$.

Definition 24 (Syntactically held locks).

$$
\begin{aligned}
\text { synlocks } \text { skip } & =\emptyset \\
\text { synlocks }(Y:=x) & =\emptyset \\
\text { synlocks }(x:=Y) & =\emptyset \\
\text { synlocks }(x:=a) & =\emptyset \\
\text { synlocks } \text { fence } & =\emptyset \\
\text { synlocks }(\text { fork } c) & =\text { synlocks } c \\
\text { synlocks }(\text { sync } \ell \text { do } c) & =\text { synlocks } c \\
\text { synlocks }(\text { holding } \ell \text { do } c) & =\{\ell \cup \cup \text { synlocks } c \\
\text { synlocks }\left(c_{1} ; c_{2}\right) & =\text { synlocks } c_{1} \cup \text { synlocks } c_{2} \\
\text { synlocks }\left(\text { if } b \text { do } c_{1} \text { else } c_{2}\right) & =\text { synlocks } c_{1} \cup \text { synlocks } c_{2} \\
\text { synlocks }(\text { while } b \text { do } c) & =\text { synlocks } c \\
\text { synlocks }\langle L, c\rangle & =\text { synlocks } c
\end{aligned}
$$

Lemma 87. Suppose active $\mathcal{E}$ and wellStruct $t$ where synlocks $\left.t \subseteq t\right|_{\Gamma, \text { high }}$ and high; wt; $\Gamma \vdash^{\mathrm{wb}} t$. Also suppose that $\left.t\right|_{\Gamma, \text { high }}$ and $\left.G\right|_{\Gamma, \text { high }}$ partition Lock $\left.\right|_{\Gamma, \text { high }}$. Then $\mathcal{T}::(G, \mathcal{E}[t] \| \mathcal{o}) \Longrightarrow^{\text {tso* }}\left(G^{\prime}, \mathcal{E}\left[\left\langle L^{\prime}\right.\right.\right.$, skip $\left.\left.\rangle\right] \| P^{\prime}\right)$ where quiet $_{\Gamma} \mathcal{T}$ and FrontReapFree $\mathcal{T}$, and where $\left.L^{\prime}\right|_{\Gamma, \text { high }}$ and $\left.G^{\prime}\right|_{\Gamma \text {, high }}$ partition Lock $\left.\right|_{\Gamma, \text { high }}$ and where $\left.L^{\prime}\right|_{\Gamma, \text { high }}=\left.\left.t\right|_{\Gamma, \text { high }} \backslash($ synlocks $t)\right|_{\Gamma, \text { high }}$ and $\left.P^{\prime}\right|_{\Gamma, \text { high }}=\emptyset$. Finally, if $w t=$ high then $L^{\prime} . w b=$ nil.

Proof. Let $\langle L, c\rangle=t$. Proof is by strong induction on size $c+$ size L.wb. Proceed by inverting the typing derivation. The interesting cases are as follows:

WB-LoAD: Here $c=x:=Y$ and $h i g h \sqsubseteq \Gamma(x)$. Construct a trace showing $(G, \mathcal{E}[t] \| \mathcal{o}) \Longrightarrow^{\mathrm{sc}}(G,\langle L[x \mapsto i]$, skip $\rangle \| \mathcal{o})$.

This is FrontReapFree. The trace is quiet because $\Gamma(x)=$ high typing ensures $L \sim_{\Gamma}^{\mathrm{wb}} L[x \mapsto i]$ and because $h i g h ; w t ; \Gamma \vdash^{\text {wb }}$ skip $\Rightarrow w t$. Conclude using the induction hypothesis, which is necessary to ensure that $w t=h i g h$ implies an empty output write buffer.

Wb-Store, wb-Eval: Similar to Wb-LoAd. . 
WB-SYNC: Here $c=$ sync $\ell$ do $c_{0}$ with high $\sqsubseteq \Gamma(\ell)=$ high and high $\sqsubseteq$ wt $=$ high and high; high; $\Gamma \vdash^{\text {wb }} c \Rightarrow$ ut.

Suppose $L=(X:=i):: L_{0}$. Because $w t=h i g h$ we have that $\Gamma(X)=$ high. Thus $(G, \mathcal{E}[t]) \Longrightarrow{ }^{\text {tso }}\left(G[X \mapsto i], \mathcal{E}\left[\left\langle L_{0}, c\right\rangle\right]\right)$. This step is FrontReapFree and quiet because only the "high components" of $L$ and $G$ are modified. Conclude using the induction hypothesis to find a FrontReapFree and quiet trace witnessing $\left(G[X \mapsto i], \mathcal{E}\left[\left\langle L_{0}, c\right\rangle\right]\right) \Longrightarrow{ }^{\text {tso* }}\left(G^{\prime}, \mathcal{E}\left[\left\langle L^{\prime}\right.\right.\right.$, skip $\left.\left.\rangle\right]\right)$ for an appropriate $L^{\prime}$ and $G^{\prime}$.

Suppose instead that $L . w b=n i l$ and $\ell \in L$. Reduce $\mathcal{E}[\langle L, c\rangle]$ to $\mathcal{E}\left[\left\langle L\right.\right.$, fence; $\left(c_{0}\right.$; fence $\left.\left.)\right\rangle\right]$ and conclude by invoking the induction hypothesis.

Finally, if $L . w b=n i l$ and $\ell \notin L$ then reduce $\mathcal{E}[\langle L, c\rangle]$ to $\mathcal{E}\left[\left\langle L \cup\{\ell\}\right.\right.$, holding $\ell$ do $\left.\left.c_{0}\right\rangle\right]$ and use the induction hypothesis to build a quiet and FrontReapFree derivation of

$$
(G, \mathcal{E}[t]) \Longrightarrow{ }^{\text {tso }}\left(G \backslash\{\ell\}, \mathcal{E}\left[\left\langle L \cup\{\ell\}, \text { holding } \ell \text { do } c_{0}\right\rangle\right]\right) \Longrightarrow^{\text {tso* }}\left(G^{\prime}, \mathcal{E}\left[\left\langle L^{\prime}, \mathbf{s k i p}\right\rangle\right] \| P^{\prime}\right)
$$

By the induction hypothesis we have that $\left.L^{\prime}\right|_{\Gamma, \text { high }}=\left.\left.(L \cup\{\ell\})\right|_{\Gamma, \text { high }} \backslash\left(\right.$ synlocks holding $\ell$ do $\left.c_{0}\right)\right|_{\Gamma, \text { high }}$. From wellStruct $t$ it follows that synlocks $\left.c_{0}\right|_{\Gamma, \text { high }}=\emptyset$ and so $\left.L^{\prime}\right|_{\Gamma, \text { high }}=\left.L\right|_{\Gamma, \text { high }}$ as required.

WB-HoLD: Here $c=$ holding $\ell$ do $c_{0}$ with high $5(\ell)=$ high and $\Gamma(\ell) \sqsubseteq w t=$ high. As $\ell \in$ synlocks $c \subseteq$ $\left.L\right|_{\Gamma, \text { high }}$, we can define $\mathcal{E}_{0}=\left(\lambda \cup\{\ell\}, \mathcal{C}\left[\right.\right.$ holding $\ell$ do [.]]) where $(\lambda, \mathcal{C})=\mathcal{E}$ and active $\mathcal{E}^{\prime}$. Inverting the typing relation shows $w t=\Gamma(\ell)=$ high and high; high; $\Gamma \vdash^{\text {wb }}\left\langle L, c_{0}\right\rangle$. By the induction hypothesis we have a quiet and FrontReapFree trace showing $\left(G, \mathcal{E}_{0}\left[\left\langle L, c_{0}\right\rangle\right]\right) \Longrightarrow{ }^{\text {tso* }}\left(G^{\prime}, \mathcal{E}_{0}\left[\left\langle L^{\prime}\right.\right.\right.$, skip $\left.\left.\rangle\right]\right)$ where $L^{\prime} . w b=n i l$. Because wellStruct $t$, it is not the case that $\ell \notin$ synlocks $c_{0}$, so $\ell \in L^{\prime}$. Finish by extending this trace using EC-HOLDRELEASE.

wB-Fence, wB-Fork: Similar to, but simpler than wB-Sync.

WB-SEQ, WB-IF, WB-WHILE: Immediate by the induction hypothesis .

Lemma 88. Suppose active $\mathcal{E}$ and wellStruct $t$ and high; wt; $\Gamma \vdash^{\mathrm{wb}} t$. Also suppose $\left.t\right|_{\Gamma, \text { high }}=\emptyset$ and

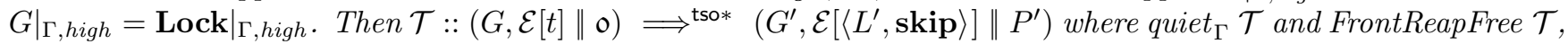
and where $\left.L^{\prime}\right|_{\Gamma, \text { high }}=\left.P^{\prime}\right|_{\Gamma, \text { high }}=\emptyset$ and $\left.G^{\prime}\right|_{\Gamma, \text { high }}=\left.\mathbf{L o c k}\right|_{\Gamma, \text { high }}$.

Proof. Immediate using Lemma 87.

Lemma 89 (WB commit step security). Suppose the following hold.

$$
\left(G_{1}, t_{1}\right) \longrightarrow{ }^{\text {commit }}\left(G_{1}^{\prime}, t_{1}^{\prime} \| \mathrm{o}\right) \quad G_{1} \sim_{\Gamma}^{\mathrm{wb}} G_{2} \quad t_{1} \sim_{\Gamma}^{\mathrm{wb}} t_{2}
$$

Then $\left(G_{2}, t_{2}\right) \Longrightarrow{ }^{\mathrm{tso} *}\left(G_{2}^{\prime}, t_{2}^{\prime}\right)$ where $\left(G_{1}^{\prime}, t_{1}^{\prime}\right) \sim_{\Gamma}^{\mathrm{wb}}\left(G_{2}^{\prime}, t_{2}^{\prime}\right)$ and both $t_{2}^{\prime}$.locks $=t_{2}$.locks and $G_{2}^{\prime}$.locks $=$ $G_{2}$. locks.

Proof. Suppose that the $\longrightarrow{ }^{\text {commit }}$ operation commits write $X:=i$. Consider the case where $\Gamma(X)=h i g h$. We can conclude immediately, taking $t_{2}^{\prime}=t_{2}$ and $G_{2}^{\prime}=G_{2}$.

Suppose instead that $\Gamma(X)=$ low. By the definition of $\sim$ wb we have that $L_{2} \cdot w b=W_{21}+(X:=i)+W_{22}$ where for each $(Y:=j) \in W_{21}$, it is the case $\Gamma(Y)=$ high. Let $n$ denote the number of writes in $W_{21}$ and define $t_{2}^{\prime}$ and $G_{2}^{\prime}$ by taking $n+1$ commit steps.

Lemma 90 (WB Eval step security). Suppose the following hold.

$$
\begin{array}{rrrr}
\left(G_{1}, t_{1}\right) \longrightarrow{ }^{\text {eval }}\left(G_{1}^{\prime}, P_{1}^{\prime}\right) & p c ; \text { wt } ; \Gamma \vdash^{\mathrm{wb}} t_{1} & \text { wellStruct } t_{1} \\
G_{1} \sim_{\Gamma}^{\mathrm{wb}} G_{2} & t_{1} \sim_{\Gamma}^{\mathrm{wb}} t_{2} & \left.t_{2}\right|_{\Gamma, \text { high }}=\emptyset & \left.G_{2}\right|_{\Gamma, \text { high }}=\left.\mathbf{L o c k}\right|_{\Gamma, \text { high }}
\end{array}
$$

Then there exist $G_{2}^{\prime}$ and $P_{2}^{\prime}$ such that $\left(G_{1}^{\prime}, P_{1}^{\prime}\right) \sim_{\Gamma}^{\mathrm{wb}}\left(G_{2}^{\prime}, P_{2}^{\prime}\right)$ and $\left(G, t_{2}\right) \Longrightarrow{ }^{\text {tso* }}\left(G_{2}^{\prime}, P_{2}^{\prime}\right)$. Furthermore $\left.P_{2}^{\prime}\right|_{\Gamma, \text { high }}=\emptyset$ and $\left.G_{2}^{\prime}\right|_{\Gamma, \text { high }}=\left.\mathbf{L o c k}\right|_{\Gamma, \text { high }}$.

Proof. We strengthen the induction hypothesis as follows. Whenever $P_{1}^{\prime}=t_{1}^{\prime} \| P_{10}^{\prime}$ there exist $\mathcal{T}, t_{2}^{\prime}$ and $P_{20}^{\prime}$ where $\mathcal{T}::\left(G_{2}, t_{2}\right) \Longrightarrow{ }^{\text {tso* }}\left(G_{2}^{\prime}, P_{2}^{\prime}\right)$ and FrontReapFree $\mathcal{T}$ and $P_{2}^{\prime}=t_{2}^{\prime} \| P_{20}^{\prime}$ and where both $t_{1}^{\prime} \sim \Gamma{ }_{\Gamma}^{\text {wb }} t_{2}^{\prime}$ and $P_{10}^{\prime} \sim_{\Gamma}^{\text {wb }} P_{20}^{\prime}$. Proceed with strong induction on quantity $\left(\right.$ size $\left(t_{1} . c m d\right)+$ size $\left.\left(t_{2} . c m d\right)\right)$. Invert $t_{1} \sim \Gamma$ wb $t_{1}$ using Lemma 85 to get three cases. Subcases of (i) will occasionally be completed by "falling through" to (ii). 
(i) $t_{1}=\left\langle L_{1}, c\right\rangle$ and $t_{2}=\left\langle L_{2}, c\right\rangle$ where $L_{1} \sim_{\Gamma}^{\mathrm{wb}} L_{2}$. Continue by inverting the $\longrightarrow{ }^{\text {commit }}$ derivation.

EC-Store: Here $c=X:=y$. If $\Gamma(y)=$ low, the definition of $\sim_{\Gamma}^{\text {wb }}$ shows $L_{1}(y)=L_{2}(y)$ and we conclude using Lemma 72. Otherwise $\Gamma(y)=h i g h$, inverting typing rule wB-STORE gives $\Gamma(X)=$ high, and the result follows from Lemma 73.

EC-LOAD: Here $c=x:=Y$. If $\Gamma(x)=$ high we conclude as updating $L_{1}$ and $L_{2}$ is not observable. If instead $\Gamma(x)=$ low then typing ensures $\Gamma(Y)=$ low and equivalences $L_{1} \sim_{\Gamma}^{\mathrm{wb}} L_{2}$ and $G_{1} \sim_{\Gamma}^{\mathrm{wb}} G_{2}$, ensures we're writing identical values to $x$.

EC-EvalExP: Follows from Lemmas 70 and 71.

EC-SyncACQUiRe: Here $c=\operatorname{sync} \ell$ do $c_{0}$ and both $L_{1} . w b=n i l$ and $\ell \in G_{1}$. First suppose that $\Gamma(\ell)=$ high. Let $\mathcal{E}=\left(\left.L_{1}\right|_{\Gamma, \text { low }},[\cdot]\right)$ and note that $t_{1}=\mathcal{E}\left[\left\langle\left. L_{1} \backslash L_{1}\right|_{\Gamma, \text { low }}, c\right\rangle\right]$. Inverting typing rule WB-SYNC shows that $h i g h ; w t ; \Gamma \vdash^{\mathrm{wb}} c_{0} \Rightarrow u t$ for some $w t$ and $u t$; using WB-SYNC, and noting that $\left.L_{1} \backslash L_{1}\right|_{\Gamma, \text { low }}$ has both an empty write buffer and no low locks, lets us find high; wt; $\Gamma \vdash^{\text {wb }}$ $\left\langle\left. L_{1} \backslash L_{1}\right|_{\Gamma, \text { low }}, c\right\rangle$. Continue by falling through to case (ii).

Now suppose $\Gamma(\ell)=$ low. Here we will use that, from inversion, $G_{1}^{\prime}=G_{1} \backslash\{\ell\}$ and $L_{1}^{\prime}=L_{1} \cup\{\ell\}$. By the definition of $\sim^{\text {wb }}$ and fact $L_{1} \cdot w b=n i l$, we see that each write $(X:=i)$ in $L_{2} . w b$ has $\Gamma(X)=$ high. We can construct a derivation showing $\left(G_{2}, t_{2}\right) \Longrightarrow^{\text {tso* }}\left(G_{20}^{\prime},\left\langle L_{20}^{\prime}, c\right\rangle\right)$ where $\left(G_{2}, t_{2}\right) \sim_{\Gamma}^{\text {wb }}\left(G_{20}^{\prime},\left\langle L_{20}^{\prime}, c\right\rangle\right)$, using finitely many $\longrightarrow^{\text {commit }}$ steps, each committing high variables. Applying the definition of $\sim^{\text {wb }}$ gives $\ell \in G_{20}^{\prime}$ and $L_{20}^{\prime} . w b=n i l$. Taking an EC-SyncAcquire step gives derivation $\left(G_{2}, t_{2}\right) \Longrightarrow^{\text {tso* }}\left(G_{20}^{\prime} \backslash\{\ell\},\left\langle L_{20}^{\prime} \cup\{\ell\}, c_{0}\right\rangle\right)$. We take this result state to be $\left(G_{2}^{\prime}, P_{2}^{\prime}\right)$ and observe that $\left.P_{2}^{\prime}\right|_{\Gamma, \text { high }}=\left.\left(L_{20}^{\prime} \cup\{\ell\}\right)\right|_{\Gamma, \text { high }}=\left.\left(L_{2} \cup\{\ell\}\right)\right|_{\Gamma, \text { high }}=\emptyset$. It suffices to show $G_{20}^{\prime} \backslash\{\ell\} \sim_{\Gamma}^{\text {wb }} G_{1}^{\prime} \backslash\{\ell\}$, which follows from Lemmas 65 and 68, and $L_{20}^{\prime} \cup\{\ell\} \sim_{\Gamma}^{\text {wb }} L_{1}^{\prime} \cup\{\ell\}$, which follows from Lemmas 65 and 69 .

eC-Fence, ec-Fork, EC-HoldRelease: Similar to the EC-SyncAcquire case.

EC-SyncReEnter: Here $c=$ sync $\ell$ do $c_{0}$. If $\Gamma(\ell)=$ high then using an argument to similar to the EC-SYNACQUIRE case fall through to (ii). If $\Gamma(\ell)=$ low then by $\sim^{\text {wb }} t_{1}$ and $t_{2}$ transition in lockstep and the case is trivial.

EC-HoldStep: Here $c=$ holding $\ell$ do $c_{0}$.

Suppose that $\Gamma(\ell)=h i g h$. As in case EC-SYNCACQUiRE we fall through to case (ii).

Suppose instead that $\Gamma(\ell)=$ low. Let $t_{10}=\left\langle L_{1}, c_{0}\right\rangle$ and $t_{20}=\left\langle L_{2}, c_{0}\right\rangle$ as well as $\mathcal{E}=$ $(\{\ell\}$, holding $\ell$ do $[\cdot])$. Inverting the evaluation relation gives $\ell \in L_{1}$ and $P_{1}^{\prime}=\mathcal{E}\left[t_{10}^{\prime}\right] \| P_{10}^{\prime}$ and $\left(G_{1}, t_{10}\right) \longrightarrow^{\text {eval }}\left(G_{1}^{\prime}, t_{10}^{\prime}\right)$. Applying the induction hypothesis to this eval step, using Lemma 77 to establish $p c ; w t ; \Gamma \vdash^{\mathrm{wb}} t_{10}$. This yields, among other properties,

$$
\mathcal{T}::\left(G_{2}, t_{20}\right) \Longrightarrow^{\text {tso* }}\left(G_{2}^{\prime}, t_{20}^{\prime}\right)
$$

where FrontReapFree $\mathcal{T}$. Take $P_{2}^{\prime}$ to be $\mathcal{E}\left[t_{20}^{\prime}\right] \| P_{20}^{\prime}$ and finish by applying Lemma 75 . .

eC-SeqStruct: Similar to EC-HoldStep.

EC-SEQSKIP: Immediate.

EC-IfTRue: Here $c=$ if $b$ do $c_{t}$ else $c_{f}$ where $L_{1}[b] \Downarrow$ true and both $P_{1}^{\prime}=\left\langle L_{1}, c_{t}\right\rangle$ and $G_{1}^{\prime}=G_{1}$.

Suppose it's not the case that $\Gamma \vdash b$ : low. Then inverting the typing relation shows both high;wt; $\Gamma \vdash^{\mathrm{wb}} c_{t} \Rightarrow u t_{t}$ and high;wt; $\Gamma \vdash^{\mathrm{wb}} c_{f} \Rightarrow u t_{f}$, as well as $w t ; \Gamma \vdash^{\mathrm{wb}} L_{1} . w b$. Without loss of generality assume $L_{2}[b] \Downarrow$ false and let $\left(G_{2}^{\prime}, P_{2}^{\prime}\right)=\left(G_{2}^{\prime},\left\langle L_{2}, c_{f}\right\rangle\right)$. It suffices to show that $\left\langle L_{1}, c_{t}\right\rangle \sim_{\Gamma}^{\mathrm{wb}}\left\langle L_{2}, c_{f}\right\rangle$, which is a consequence of Lemma 80.

Suppose instead that that $\Gamma \vdash b$ : low. Lemma 84 shows $L_{2}[b] \Downarrow$ true so $\left(G_{2}, t_{2}\right) \Longrightarrow{ }^{\text {tso* }}\left(G_{2},\left\langle L_{2}, c_{t}\right\rangle\right) \sim_{\Gamma}^{\text {wb }}$ $\left(G_{1},\left\langle L_{1}, c_{t}\right\rangle\right)=\left(G_{1}^{\prime}, P_{1}^{\prime}\right)$.

ec-IfFalse, ec-WhileTrue, ec-WhileFalse: Similar to, or simpler than, ec-IfTrue. EC-REAP Trivial. .

(ii) $t_{1}=\mathcal{E}\left[\left\langle L_{1}, c_{1}\right\rangle\right]$ where $c_{1} \neq$ skip and $h i g h ; w t_{1} ; \Gamma \vdash^{\text {wb }}\left\langle L_{1}, c_{1}\right\rangle$ for some $w t_{1}$. By transitivity (Lemma 65) it suffices to show $\left(G_{1}^{\prime}, P_{1}^{\prime}\right) \sim_{\Gamma}^{\text {wb }}\left(G_{1}, t_{1}\right)$. Conclude via Lemma 81.

(iii) $t_{1}=\mathcal{E}\left[\left\langle L_{1}\right.\right.$, skip $\left.\rangle\right]$ and $t_{2}=\mathcal{E}\left[\left\langle L_{2}, c_{2}\right\rangle\right]$. We know the following for some $w t_{0}$.

$c_{2} \neq$ skip $\quad L_{1} \sim_{\Gamma}^{\mathrm{wb}} L_{2} \quad$ active $\mathcal{E} \quad$ high; $w t_{0} ; \Gamma \vdash^{\mathrm{wb}}\left\langle L_{1}, c_{1}\right\rangle \quad$ high;wt $; \Gamma \vdash^{\mathrm{wb}}\left\langle L_{2}, c_{2}\right\rangle$ 
By Lemma 88, we have $\mathcal{T}::\left(G, \mathcal{E}\left[\left\langle L_{2}, c_{2}\right\rangle\right] \| \mathcal{o}\right) \Longrightarrow^{\text {tso* }}\left(G_{2}^{\prime}, \mathcal{E}\left[\left\langle L_{2}^{\prime}\right.\right.\right.$, skip $\left.\left.\rangle\right] \| P_{2}^{\prime}\right)$ where quiet $_{\Gamma} \mathcal{T}$, FrontReapFree $\mathcal{T}$, and $\left.L_{2}^{\prime}\right|_{\Gamma, \text { high }}=\left.P_{2}^{\prime}\right|_{\Gamma, \text { high }}=\emptyset$. By Lemmas 65 and 86 we find:

$$
\begin{aligned}
\mathcal{E}\left[\left\langle L_{2}^{\prime}, \text { skip }\right\rangle\right] & \sim_{\Gamma}^{\mathrm{wb}} \mathcal{E}\left[\left\langle L_{2}, c_{2}\right\rangle\right] \sim_{\Gamma}^{\mathrm{wb}} t_{1} \\
G_{2}^{\prime} & \sim_{\Gamma}^{\mathrm{wb}} G_{2} \sim_{\Gamma}^{\mathrm{wb}} G_{1} \\
& P_{2}^{\prime} \sim_{\Gamma}^{\mathrm{wb}} \circ
\end{aligned}
$$

Because $c_{2} \neq$ skip it is the case that size $\left(\mathcal{E}\left[\left\langle L_{2}^{\prime}\right.\right.\right.$, skip $\left.\left.\rangle\right] . c m d\right)<$ size $\left(\mathcal{E}\left[\left\langle L_{2}, c_{2}\right\rangle\right] . c m d\right)$, so we can use the induction hypothesis to find $G_{2}^{\prime \prime}$ and $P_{2}^{\prime \prime}$ such that $\left(G_{1}^{\prime}, P_{1}^{\prime}\right) \sim_{\Gamma}^{\text {wb }}\left(G_{2}^{\prime \prime}, P_{2}^{\prime \prime}\right)$ and $\left(G_{2}^{\prime}, \mathcal{E}\left[\left\langle L_{2}^{\prime}\right.\right.\right.$, skip $\left.\left.\rangle\right]\right) \Longrightarrow{ }^{\text {tso* }}\left(G_{2}^{\prime \prime}, P_{2}^{\prime \prime}\right)$. By Lemma $66,\left(G_{1}^{\prime}, P_{1}^{\prime}\right) \sim_{\Gamma}^{\mathrm{wb}}\left(G_{2}^{\prime \prime}, P_{2}^{\prime \prime} \| P_{2}^{\prime}\right)$. Thus it suffices to show $\left.\left(P_{2}^{\prime \prime} \| P_{2}^{\prime}\right)\right|_{\Gamma \text {, high }}=\emptyset$, which is immediate, and $\left(G_{2}, t_{2}\right) \Longrightarrow{ }^{\text {tso* }}\left(G_{2}^{\prime \prime}, P_{2}^{\prime \prime} \| P_{2}^{\prime}\right)$, which is a consequence of Lemma 3.

Theorem 3 (WB Security). Suppose $\left(G_{1}, P_{1}\right) \sim_{\Gamma}^{\mathrm{wb}}\left(G_{2}, P_{2}\right)$ and $\overline{p c} ; \overline{w t} ; \Gamma \vdash^{\mathrm{wb}} P_{1}$ and wellStruct $P_{1}$. Suppose also that $\left(G_{1}, P_{1}\right) \Longrightarrow^{\text {tso }}\left(G_{1}^{\prime}, P_{1}^{\prime}\right)$. Furthermore $\left.P_{2}\right|_{\Gamma, \text { high }}=\emptyset$ and $\left.G_{2}\right|_{\Gamma, \text { high }}=\left.\mathbf{L o c k}\right|_{\Gamma, \text { high }}$. Then there exists $G_{2}^{\prime}, P_{2}^{\prime}$ such that $\left(G_{1}^{\prime}, P_{1}^{\prime}\right) \sim_{\Gamma}^{\text {wb }}\left(G_{2}^{\prime}, P_{2}^{\prime}\right)$ and $\left(G_{2}, P_{2}\right) \Longrightarrow{ }^{\text {tso* }}\left(G_{2}^{\prime}, P_{2}^{\prime}\right)$, and both $\left.P_{2}^{\prime}\right|_{\Gamma, \text { high }}=\emptyset$ and $\left.G_{2}^{\prime}\right|_{\Gamma, \text { high }}=\left.\mathbf{L o c k}\right|_{\Gamma, \text { high }}$.

Proof. Inverting the tso-evaluation relation and appealing to Lemma 76 gives

$$
\begin{aligned}
& P_{1}=P_{11}\left\|t_{1}\right\| P_{12} \\
& P_{2}=P_{21}\left\|P_{2}^{*}\right\| P_{22} \\
& P_{1}^{\prime}=P_{11}\left\|Q_{1}^{\prime}\right\| P_{12}
\end{aligned}
$$

where $P_{2}^{*}$ contains at most one thread (i.e., $P_{2}^{*} \in\left\{\mathfrak{o}, t_{2} \| \mathcal{o}\right\}$ for some $t_{2}$ ) and the following hold:

$$
\begin{gathered}
\left(G, t_{1}\right) \longrightarrow^{o p}\left(G_{1}^{\prime}, Q_{1}^{\prime}\right) \\
P_{11} \sim_{\Gamma}^{\mathrm{wb}} P_{21} \\
t \| \mathcal{O} \sim_{\Gamma}^{\mathrm{wb}} P_{2}^{*} \\
P_{12} \sim_{\Gamma}^{\mathrm{wb}} P_{22}
\end{gathered}
$$

It suffices to show that there exists $G_{2}^{\prime}$ and $Q_{2}^{\prime}$ such that $\left(G_{1}^{\prime}, Q_{1}^{\prime}\right) \sim_{\Gamma}^{\text {wb }}\left(G_{2}^{\prime}, Q_{2}^{\prime}\right)$ and $\left(G_{2}, P_{2}^{*}\right) \Longrightarrow^{\text {tso* }}\left(G_{2}^{\prime}, Q_{2}^{\prime}\right)$. (Observe that while we could rename threads in $Q_{2}^{\prime}$, we do not need to; thread names are only really relevant for the data-race freedom argument.) Inspecting the definition of $\sim_{\Gamma}^{\text {wb }}$ shows there are only three ways in which to find $t \| \mathcal{o} \sim_{\Gamma}^{\mathrm{wb}} P_{2}^{*}$. Proceed by case analysis.

First suppose that that the equivalence arises from Definition 21, clause 5b. Here high; high; $\Gamma \vdash^{\text {wb }} t_{1}$ and via Lemma $83,\left(G_{1}, t \| \mathrm{o}\right) \sim_{\Gamma}^{\mathrm{wb}}\left(G_{1}^{\prime}, Q_{1}^{\prime}\right)$. Conclude using Lemma 65 , which states $\sim^{\text {wb }}$ is an equivalence relation, and taking $G_{2}$ and $P_{2}^{*}$ as existential witnesses $G_{2}^{\prime}$ and $Q_{2}^{\prime}$.

Second suppose that that the equivalence arises from definition 21, clause 5c. Here $P_{2}^{*}=t_{2} \| \mathcal{o}$ where high; high; $\Gamma \vdash^{\mathrm{wb}} t_{2}$ and $t_{1} \sim_{\Gamma}^{\mathrm{wb}} \mathrm{o}$. From $t_{1} \sim_{\Gamma}^{\mathrm{wb}} \mathrm{o}$ it follows that high; high; $\Gamma \vdash^{\text {wb }} t_{1}$. Again taking $G_{2}$ and $P_{2}^{*}$ to be witnesses $G_{2}^{\prime}$ and $Q_{2}^{\prime}$ conclude with the following equational reasoning:

$$
\begin{array}{rlll}
\left(G^{\prime}, Q_{1}^{\prime}\right) & \sim_{\Gamma}^{\text {wb }} & \left(G_{1}, t_{1} \| \mathrm{o}\right) & \text { by Lemma } 83 \\
& \sim_{\Gamma}^{\text {wb }} & \left(G_{1}, \mathcal{o}\right) & \\
& \sim_{\Gamma}^{\text {wb }} & \left(G_{2}, \mathcal{o}\right) & \text { by assumption } \\
& \sim_{\Gamma}^{\text {wb }} & \left(G_{2}, t_{2} \| \mathrm{o}\right) & \\
& =\left(G_{2}, P_{2}^{*}\right) &
\end{array}
$$

Third suppose that that the equivalence arises from Definition 21, clause 5d. Here $P_{2}^{*}=t_{2} \|$ o for some $t_{2}$ with $t_{1} \sim_{\Gamma}^{\text {wb }} t_{2}$. Finitely many inversions of the typing relation show $p c ; w t ; \Gamma \vdash^{\text {wb }} t_{1}$ for some $p c$ and $w t$. Similarly wellStruct $t_{1}$ and $\left.t_{2}\right|_{\Gamma, \text { high }}=\emptyset$. Conclude via Lemmas 89 and 90 .

Corollary 5. Suppose $\left(G_{1}, P_{1}\right) \sim_{\Gamma}^{\mathrm{wb}}\left(G_{2}, P_{2}\right)$ and $\overline{p c} ; \overline{w t} ; \Gamma \vdash^{\mathrm{wb}} P_{1}$ and wellStruct $P_{1}$. Suppose also that $\left(G_{1}, P_{1}\right) \Longrightarrow^{\text {tso* }}\left(G_{1}^{\prime}, P_{1}^{\prime}\right)$. Furthermore $\left.P_{2}\right|_{\Gamma, \text { high }}=\emptyset$ and $\left.G_{2}\right|_{\Gamma, \text { high }}=\left.\mathbf{L o c k}\right|_{\Gamma, \text { high }}$. Then there exist $G_{2}^{\prime}$ and $P_{2}^{\prime}$ such that $\left(G_{1}^{\prime}, P_{1}^{\prime}\right) \sim_{\Gamma}^{\mathrm{wb}}\left(G_{2}^{\prime}, P_{2}^{\prime}\right)$ and $\left(G_{2}, P_{2}\right) \Longrightarrow^{\text {tso* }}\left(G_{2}^{\prime}, P_{2}^{\prime}\right)$ and $\left.P_{2}^{\prime}\right|_{\Gamma, \text { high }}=\emptyset$.

Proof. By finitely many application of Theorem 3 and Lemmas 5 and 64 . 
Corollary 6. Suppose $G_{1} \sim_{\Gamma}^{\mathrm{wb}} G_{2}$ and $p c ; w t ; \Gamma \vdash^{\mathrm{wb}} c \Rightarrow$ ut and src c. Also assume $\left.G_{2}\right|_{\Gamma, \text { high }}=\left.\mathbf{L o c k}\right|_{\Gamma, \text { high }}$. If $\left(G_{1},\left\langle L_{\oslash}, c\right\rangle\right) \Longrightarrow^{\text {tso* }}\left(G_{1}^{\prime}, \mathcal{o}\right)$ then $\left(G_{2},\left\langle L_{\oslash}, c\right\rangle\right) \Longrightarrow^{\text {tso* }}\left(G_{2}^{\prime}, \mathcal{o}\right)$ for some $G_{2}^{\prime}$ where $G_{1}^{\prime} \sim_{\Gamma}^{\text {wb }} G_{2}^{\prime}$.

Proof. An instantiation of the first corollary of Theorem 3 shows $\left(G_{2}, t\right)$ evaluates to a configuration related to $\left(G_{1}^{\prime}, \mathrm{o}\right)$, and preservation (Lemma 64 ) and Lemmas 5,9 , and 88, show this evaluates to pool $\mathrm{o}$.

Corollary 7 (WB Simple possibilistic noninterference). Suppose $p c ; w t ; \Gamma \vdash^{\mathrm{wb}} c \Rightarrow$ ut and src $c$. Then $c$ is possibilistically noninterfering under tso and $\Gamma$.

\section{Expressive typing for SC programs}

\subsection{Typing}

As above:

$$
\text { Static Security Context } \Gamma::=\text { HeapVar } \cup \text { LocalVar } \cup \text { Lock } \rightarrow \tau
$$

The type system will borrow a shared notion of $\vdash^{\text {wb }}$ for locks and write buffers. Note that while we wrote $w t ; \Gamma \vdash^{\text {wb }} W$ earlier, we will write $p c ; \Gamma \vdash^{\text {wb }} W$ here. This is okay because both $w t$ and $p c$ are metavariables ranging over labels.

$$
\begin{aligned}
& p c ; \Gamma \vdash^{\mathrm{sc}} c \\
& \frac{p c \sqcup \Gamma(Y) \sqsubseteq \Gamma(x)}{p c ; \Gamma \vdash^{\mathrm{sc}} x:=Y} \text { SC-LOAD } \quad \frac{p c \sqcup \Gamma(y) \sqsubseteq \Gamma(X)}{p c ; \Gamma \vdash^{\mathrm{sc}} X:=y} \text { SC-StORE } \quad \frac{\Gamma \vdash a: \tau \quad p c \sqcup \tau \sqsubseteq \Gamma(x)}{p c ; \Gamma \vdash^{\mathrm{sc}} x:=a} \text { SC-EvaL } \\
& \frac{p c \sqsubseteq \Gamma(\ell) \quad \Gamma(\ell) ; \Gamma \vdash^{\mathrm{sc}} c}{p c ; \Gamma \vdash^{\mathrm{sc}} \text { sync } \ell \text { do } c} \text { SC-SynC } \quad \frac{p c \sqsubseteq \Gamma(\ell) \quad \Gamma(\ell) ; \Gamma \vdash^{\mathrm{sc}} c}{p c ; \Gamma \vdash^{\mathrm{sc}} \text { holding } \ell \text { do } c} \text { SC-Hold } \quad \frac{}{p c ; \Gamma \vdash^{\mathrm{sc}} \text { fence }} \text { SC-FenCE } \\
& \frac{p c ; \Gamma \vdash^{\mathrm{sc}} c}{p c ; \Gamma \vdash^{\mathrm{sc}} \text { fork } c} \text { SC-ForK } \quad \frac{p c ; \Gamma \vdash^{\mathrm{sc}} c_{1} \quad p c ; \Gamma \vdash^{\mathrm{sc}} c_{2}}{p c ; \Gamma \vdash^{\mathrm{sc}} c_{1} ; c_{2}} \mathrm{SC}-\mathrm{SEQ} \\
& \frac{\Gamma \vdash b: \tau \quad p c \sqcup \tau ; \Gamma \vdash^{\mathrm{sc}} c_{1} \quad p c \sqcup \tau ; \Gamma \vdash^{\mathrm{sc}} c_{2}}{p c ; \Gamma \vdash^{\mathrm{sc}} \text { if } b \text { do } c_{1} \text { else } c_{2}} \mathrm{SC- \textrm {IF }} \quad \frac{\Gamma \vdash b: l o w \quad p c ; \Gamma \vdash^{\mathrm{sc}} c}{l o w ; \Gamma \vdash^{\mathrm{sc}} \text { while } b \text { do } c} \text { SC-WhILE } \\
& \overline{p c ; \Gamma \vdash^{\mathrm{sc}} \text { skip }} \text { SC-SKIP }
\end{aligned}
$$

$p c ; \Gamma \vdash^{\mathrm{sc}} t$

$$
\frac{p c ; \Gamma \vdash^{\mathrm{wb}} \lambda \quad p c ; \Gamma \vdash^{\mathrm{wb}} W \quad p c ; \Gamma \vdash^{\mathrm{sc}} c}{p c ; \Gamma \vdash^{\mathrm{sc}}\langle(M, \lambda, W), c\rangle_{\iota}}
$$

$\overline{p c} ; \Gamma \vdash^{\mathrm{sc}} P$

$$
\overline{. ; \Gamma \vdash^{\mathrm{sc}} \mathrm{o}} \quad \frac{p c ; \Gamma \vdash^{\mathrm{sc}} t \quad \overline{p c} ; \Gamma \vdash^{\mathrm{sc}} P}{p c, \overline{p c} ; \Gamma \vdash^{\mathrm{sc}} t \| P}
$$

Lemma 91 (SC Admissibility of subtyping). Suppose $p c_{1} \sqsubseteq p c_{2}$. Then the following hold.

(i) $p c_{2} ; \Gamma \vdash^{\mathrm{sc}} p c_{1}$ implies $p c_{1} ; \Gamma \vdash^{\mathrm{sc}} p c_{1}$

(ii) $p c_{2} ; \Gamma \vdash^{\mathrm{sc}} t$ implies $p c_{1} ; \Gamma \vdash^{\mathrm{sc}} t$. 
Proof. Statement (i) follows from easy inductions on the typing derivations. Statement (ii) follows from (i) and Lemma 57.

Lemma 92. If $p c ; \Gamma \vdash^{\mathrm{sc}} \mathcal{E}[t]$ then $p c ; \Gamma \vdash^{\mathrm{sc}} t$.

Proof. Let $\mathcal{E}=(\lambda, \mathcal{C})$. First use induction on the size of $\lambda$ to show $p c ; \Gamma \vdash^{\text {sc }} \mathcal{E}_{0}[t]$ where $\mathcal{E}_{0}=(\emptyset, \mathcal{C})$. Conclude by an easy structural induction on $\mathcal{C}$, using Lemma 57 .

Lemma 93. Suppose that high; $\Gamma \vdash^{\mathrm{sc}} t$. Then t.cmd.locks $\subseteq$ Lock $\left.\right|_{\Gamma, \text { high }}$.

Proof. by trivial induction on the typing derivation.

Lemma 94 (SC Commit Step Preservation). Suppose $p c ; \Gamma \vdash^{\text {sc }} t$ and $(G, t) \longrightarrow^{\text {commit }}\left(G^{\prime}, P^{\prime}\right)$. Then $\overline{p c} ; \Gamma ; P^{\prime} \vdash^{\mathrm{wb}} w$ here $p c \sqsubseteq \overline{p c}$.

Proof. Let $\langle L, c\rangle=t$. Then $P^{\prime}=\left\langle L_{0}, c\right\rangle \|$ o where $L=(X:=i)+L_{0}$ for some $X, i$, and $L_{0}$. We must show that $p c ; \Gamma \vdash^{\mathrm{wb}} L_{0}$. wb, which follows from inverting the typing derivation.

Lemma 95 (SC Eval Step Preservation). Suppose $p c ; \Gamma \vdash^{\mathrm{sc}} t$ and $(G, t) \longrightarrow^{\text {eval }}\left(G^{\prime}, P^{\prime}\right)$. Then $\overline{p c} ; \Gamma \vdash^{\mathrm{sc}} P^{\prime}$ where $p c \sqsubseteq \overline{p c}$.

Proof. Let $\langle L, c\rangle=t$ and proceed by induction on the $\longrightarrow^{\text {eval }}$ step derivation.

EC-Store: Here $c=(X:=y)$ and $P^{\prime}=\langle L+(X:=i)$, skip $\rangle \|$ o for some $i$. Inverting the typing derivation shows $p c \sqsubseteq \Gamma(X)$ and $p c ; \Gamma \vdash^{\mathrm{sc}} L . w b$. We must show $p c ; \Gamma \vdash^{\mathrm{wb}} L . w b+(X:=i)$, which follows from Lemma 61 . It remains to show $p c ; \Gamma \vdash^{\mathrm{sc}}$ skip; this is immediate.

EC-LoAd, EC-EvalExp, EC-Fence: Immediate.

EC-SYNCACQUIRE: Here $c=\operatorname{sync} \ell$ do $c_{0}$ and $P^{\prime}=\left\langle L \cup\{\ell\}\right.$, holding $\ell$ do $\left.\left.c_{0}\right)\right\rangle \|$ o. Recall that $L$. wb $=$ nil. Inverting the typing relation gives $p c \sqsubseteq \Gamma(\ell)$ and $\Gamma(\ell) ; \Gamma \vdash^{\mathrm{sc}} c_{0}$. Because the lock set changed, we must show that $p c ; \Gamma \vdash^{\mathrm{wb}}$ L.locks $\cup\{\ell\}$, which is immediate from Lemma 59. Conclude by constructing a derivation of $p c ; \Gamma \vdash^{\mathrm{sc}}$ holding $\ell$ do $c_{0}$.

EC-SyncReenter: Here $c=$ sync $\ell$ do $c_{0}$ and $P^{\prime}=\left\langle L\right.$, fence; $\left(c_{0}\right.$; fence $\left.)\right\rangle \| \mathcal{O}$. Inverting the typing relation shows $\Gamma(\ell) ; \Gamma \vdash^{\mathrm{sc}} c_{0}$ and $p c \sqsubseteq \Gamma(\ell)$ so that Lemma 91 yields a derivation showing $p c ; \Gamma \vdash^{\mathrm{sc}} c_{0}$. It remains to construct the a derivation showing $p c ; \Gamma \vdash^{\mathrm{sc}}$ fence; $\left(c_{0}\right.$; fence) .

EC-HoldSteP: Here $c=$ holding $\ell$ do $c_{0}$ and $P^{\prime}=\left\langle L^{\prime}\right.$, holding $\ell$ do $\left.c_{0}^{\prime}\right\rangle \| P_{0}^{\prime}$ where $\left(G,\left\langle L, c_{0}\right\rangle\right) \longrightarrow^{\text {eval }}$ $\left(G^{\prime},\left\langle L^{\prime}, c_{0}^{\prime}\right\rangle \| P_{0}^{\prime}\right)$. Inverting the typing derivation gives $\Gamma(\ell) ; \Gamma \vdash^{\mathrm{sc}} c_{0}$ and $p c \sqsubseteq \Gamma(\ell)$. Applying the induction hypothesis yields $\overline{p c} ; \Gamma \vdash^{\mathrm{sc}}\left\langle L^{\prime}, c_{0}^{\prime}\right\rangle \| P_{0}^{\prime}$ where $\Gamma(\ell) \sqsubseteq \overline{p c}$. It remains to show that $p c ; \Gamma \vdash^{\text {sc }}$ holding $\ell$ do $c_{0}^{\prime}$, which follows from rule SC-HoLD and Lemma 91.

EC-HoldRelease: Here $c=$ holding $\ell$ do skip and $P^{\prime}=\langle L \backslash\{\ell\}$, skip $\rangle \| \mathcal{o}$ with $L . w b=$ nil. Noting that $p c ; \Gamma \vdash^{\text {wb }} L . w b$ and that Lemma 60 gives $p c ; \Gamma \vdash^{\text {wb }}$ L.locks $\backslash\{\ell\}$, the result is immediate.

EC-ForK: Here $c=$ fork $c_{0}$ and $P^{\prime}=\langle L$, skip $\rangle\left\|\left\langle L_{\oslash}, c_{0}\right\rangle\right\|$ o and $L . w b=$ nil.

First we show that $p c ; \Gamma \vdash^{\text {sc }}\left\langle L_{\oslash}, c_{0}\right\rangle \|$ o. Inverting the typing relation gives $p c ; \Gamma \vdash^{\text {sc }} c_{0}$. Finish building easy derivations of $p c ; \Gamma \vdash^{\text {wb }} L_{\oslash}$.locks and $p c ; \Gamma \vdash^{\text {wb }} L_{\oslash}$.wb.

Second we show $p c ; \Gamma \vdash^{\mathrm{sc}}$ skip, and $p c ; \Gamma \vdash^{\text {wb }} L . w b$ both of which are immediate.

eC-SeqStruct: Here $c=c_{1} ; c_{2}$ and $P^{\prime}=\left\langle L^{\prime}, c_{1}^{\prime}\right\rangle \| P_{0}^{\prime}$ where $\left(G,\left\langle L, c_{1}\right\rangle\right) \longrightarrow^{\text {eval }}\left(G^{\prime},\left\langle L^{\prime}, c_{1}^{\prime}\right\rangle \| P_{0}^{\prime}\right)$. Inverting the typing derivation gives $p c ; \Gamma \vdash^{\mathrm{sc}} c_{1}$ and $p c ; \Gamma \vdash^{\mathrm{sc}} c_{2}$.

The induction hypothesis shows $\overline{p c} ; \Gamma \vdash^{\mathrm{sc}}\left\langle L^{\prime}, c_{1}^{\prime}\right\rangle \| P_{0}^{\prime}$. where $p c \sqsubseteq \overline{p c}$. By Lemma $91, p c ; \Gamma \vdash^{\mathrm{sc}}\left\langle L^{\prime}, c_{1}^{\prime}\right\rangle$, and we conclude by inverting this judgment and constructing constructing a derivation of $p c ; \Gamma \vdash^{\mathrm{sc}} c_{1}^{\prime} ; c_{2}$ with SC-SEQ.

EC-SeQSkiP: Here $c=\operatorname{skip} ; c_{2}$ and $P^{\prime}=\left\langle L, c_{2}\right\rangle \|$ o. Trivial by inverting the typing derivation.

eC-IfTrue, ec-IfFalse, ec-WhileTrue, eC-WhileFalse: Immediate, using Lemma 57 for eC-WhileTrue. EC-REAP: Immediate.

Lemma 96 (SC Preservation). Suppose $\overline{p c} ; \Gamma \vdash^{\mathrm{sc}} P$ and $(G, P) \Longrightarrow^{\mathrm{sc} *}\left(G^{\prime}, P^{\prime}\right)$. Then there exist $\overline{p c}^{\prime}$ such that $\overline{p c}^{\prime} ; \Gamma \vdash^{\mathrm{sc}} P^{\prime}$.

Proof. By induction on the length of the $\Longrightarrow^{\text {sc* }}$ derivation, using Lemmas 94 or 95. 


\subsection{Equivalences}

\section{Definition $25\left(\sim_{\Gamma}^{\mathrm{sc}}\right)$.}

1. $t_{1} \sim_{\Gamma}^{\mathrm{sc}} t_{2}$ is defined by the following introduction rules.

(a) $\left\langle L_{1}, c\right\rangle_{\iota_{1}} \sim_{\Gamma}^{\mathrm{sc}}\left\langle L_{2}, c\right\rangle_{\iota_{2}}$ when $L_{1} \sim_{\Gamma}^{\mathrm{wb}} L_{2}$

(b) $\mathcal{E}\left[\left\langle L_{1}, c_{1}\right\rangle_{\iota_{1}}\right] \sim_{\Gamma}^{\mathrm{sc}} \mathcal{E}\left[\left\langle L_{2}, c_{2}\right\rangle_{\iota_{2}}\right]$ when $L_{1} \sim_{\Gamma}^{\mathrm{wb}} L_{2}$ and both high; $\Gamma \vdash^{\mathrm{sc}}\left\langle L_{1}, c_{1}\right\rangle_{\iota_{1}}$ and high; $\Gamma \vdash^{\mathrm{sc}}$ $\left\langle L_{2}, c_{2}\right\rangle_{\iota_{2}}$ for some wt.

2. $P_{1} \sim_{\Gamma}^{\mathrm{sc}} P_{2}$ is defined by the least fixed point of the following implications.

(a) $\mathcal{O} \sim_{\Gamma}^{\mathrm{sc}} \mathcal{O}$, always

(b) $t \| P_{1} \sim_{\Gamma}^{\mathrm{sc}} P_{2}$ when high; $\Gamma \vdash^{\mathrm{sc}} t$ and $P_{1} \sim_{\Gamma}^{\mathrm{sc}} P_{2}$

(c) $P_{1} \sim_{\Gamma}^{\mathrm{sc}} t \| P_{2}$ when high; $\Gamma \vdash^{\mathrm{sc}} t$ and $P_{1} \sim_{\Gamma}^{\mathrm{sc}} P_{2}$

(d) $t_{1}\left\|P_{1} \sim_{\Gamma}^{\mathrm{sc}} t_{2}\right\| P_{2}$ when $t_{1} \sim_{\Gamma}^{\mathrm{sc}} t_{2}$ and $P_{1} \sim_{\Gamma}^{\mathrm{sc}} P_{2}$

3. $\left(G_{1}, P_{1}\right) \sim_{\Gamma}^{\mathrm{sc}}\left(G_{2}, P_{2}\right)$ when $G_{1} \sim_{\Gamma}^{\mathrm{wb}} G_{2}$ and $P_{1} \sim_{\Gamma}^{\mathrm{sc}} P_{2}$.

Lemma 97. Each $\sim_{\Gamma}^{\mathrm{sc}}$ relation is an equivalence relation.

Proof. by inspection.

Lemma 98. If $P_{11}\left\|t_{1}\right\| P_{12} \sim_{\Gamma}^{\text {sc }} P_{2}$ then $P_{2}=P_{21}\left\|P_{2}^{*}\right\| P_{22}$ where the following hold:

$$
\begin{aligned}
P_{21} & \sim{ }_{\Gamma}^{\mathrm{sc}} P_{11} \\
P_{2}^{*} & \sim{ }_{\Gamma}^{\mathrm{sc}} \\
P_{22} & \sim{ }_{\Gamma}^{\mathrm{sc}} P_{12} \\
P_{2}^{*} & \in\left\{\mathfrak{o}, t_{2} \| \mathfrak{o}\right\} \text { for some } t_{2}
\end{aligned}
$$

Proof. By any easy induction on the sum of the lengths of $P_{1}$ and $P_{2}$.

Lemma 99 (Strong inversion for $\sim^{\mathrm{sc}}$ ). Suppose $t_{1} \sim_{\Gamma}^{\mathrm{sc}} t_{2}$. Then at least one the following conditions holds. Either,

(i) $t_{1}=\left\langle L_{1}, c\right\rangle$ and $t_{2}=\left\langle L_{2}, c\right\rangle$ where $L_{1} \sim_{\Gamma}^{\mathrm{sc}} L_{2}$, or

(ii) $t_{1}=\mathcal{E}\left[\left\langle L_{1}, c_{1}\right\rangle\right]$ where $c_{1} \neq$ skip and high; $\Gamma \vdash^{\mathrm{sc}}\left\langle L_{1}, c_{1}\right\rangle$, or

(iii) $t_{1}=\mathcal{E}\left[\left\langle L_{1}, \mathbf{s k i p}\right\rangle\right]$ and $t_{2}=\mathcal{E}\left[\left\langle L_{2}, c_{2}\right\rangle\right]$, where the following hold:

$$
c_{2} \neq \text { skip, } \quad L_{1} \sim_{\Gamma}^{\mathrm{wb}} L_{2}, \quad \text { canEval } t_{1} \text { implies active } \mathcal{E}, \quad \text { high; } \Gamma \vdash^{\mathrm{sc}}\left\langle L_{1}, c_{1}\right\rangle \text {, and }
$$

$$
h i g h ; \Gamma \vdash^{\mathrm{sc}}\left\langle L_{2}, c_{2}\right\rangle \text {. }
$$

Proof. Suppose that $t_{1}$ and $t_{2}$ are related by Definition 25, case 1a. Conclude as (i) is satisfied.

Suppose instead that $t_{1}$ and $t_{2}$ are related by case $1 \mathrm{~b}$, so Then $t_{1}=\mathcal{E}\left[\left\langle L_{1}, c_{1}\right\rangle\right]$ and $t_{2}=\mathcal{E}\left[\left\langle L_{2}, c_{2}\right\rangle\right]$ and both high; $\Gamma \vdash^{\text {sc }}\left\langle L_{1}, c_{1}\right\rangle$ and high; $\Gamma \vdash^{\text {sc }}\left\langle L_{2}, c_{2}\right\rangle$. Additionally $L_{1} \sim_{\Gamma}^{\text {wb }} L_{2}$. If $c_{1} \neq$ skip then condition (ii) is satisfied. Suppose instead that $c_{1}=$ skip. If $c_{2}=$ skip then using Lemma 69 we see that condition (i) is satisfied. Now suppose $c_{2} \neq$ skip. If it's not the case that canEval $t_{1}$ then (iii) is satisfied and we conclude. Otherwise use Lemma 2 to find $\mathcal{E}_{0}$ where active $\mathcal{E}_{0}$ and $\mathcal{E}_{0}\left[t_{i}\right]=\mathcal{E}\left[t_{i}\right]$. Conclude as condition (iii) is satisfied.

Lemma 100. Suppose $t_{1} \sim_{\Gamma}^{\mathrm{sc}} t_{2}$. Then $\mathcal{E}\left[t_{1}\right] \sim_{\Gamma}^{\mathrm{sc}} \mathcal{E}\left[t_{2}\right]$.

Lemma 101. Suppose $L_{1} \sim_{\Gamma}^{\mathrm{wb}} L_{2}$ and high; $\Gamma \vdash^{\mathrm{sc}} c_{1}$ and high $; \Gamma \vdash^{\mathrm{sc}} c_{2}$. Suppose also that high; $\Gamma \vdash^{\mathrm{wb}} L_{1}$.wb. Then $\left\langle L_{1}, c_{1}\right\rangle \sim \sim_{\Gamma}^{\mathrm{sc}}\left\langle L_{2}, c_{2}\right\rangle$. 
Proof. By the definition of $\sim^{\text {wb }}$ we want to find $\mathcal{E}=(\lambda, \mathcal{C}), L_{10}$, and $L_{20}$, such that the following hold.

$$
\left\langle L_{1}, c_{1}\right\rangle=\mathcal{E}\left[\left\langle L_{10}, c_{1}\right\rangle\right] \quad\left\langle L_{2}, c_{2}\right\rangle=\mathcal{E}\left[\left\langle L_{20}, c_{2}\right\rangle\right] \quad h i g h ; \Gamma \vdash^{\mathrm{sc}}\left\langle L_{10}, c_{1}\right\rangle \quad h i g h ; \Gamma \vdash^{\mathrm{sc}}\left\langle L_{20}, c_{2}\right\rangle
$$

Let $\mathcal{C}=[\cdot]$ and $\lambda=\left.L_{1}\right|_{\Gamma, \text { low }}$. Let also $L_{10}=L_{1} \backslash \lambda$ and $L_{20}=L_{2} \backslash \lambda$. Clearly $\left\langle L_{1}, c_{1}\right\rangle=\mathcal{E}\left[\left\langle L_{10}, c_{1}\right\rangle\right]$. Because $L_{1} \sim_{\Gamma}^{\text {wb }} L_{2}$ it's also true that $\lambda=\left.L_{2}\right|_{\Gamma, \text { low }}$, so $\left\langle L_{2}, c_{2}\right\rangle=\mathcal{E}\left[\left\langle L_{20}, c_{2}\right\rangle\right]$. We demonstrate $h i g h ; \Gamma \vdash^{\text {sc }}\left\langle L_{10}, c_{1}\right\rangle$ by using the definition of $L_{10}$ and the premises to show that high; $\Gamma \vdash^{\mathrm{wb}} L_{10}$.locks and high; $\Gamma \vdash^{\mathrm{wb}} L_{10}$.wb. It remains to show high; wt; $\Gamma \vdash^{\text {wb }}\left\langle L_{20}, c_{2}\right\rangle$, which works as above, using Lemma 74 to help establish $h i g h ; \Gamma \vdash^{\mathrm{wb}} L_{20} . w b$.

Lemma 102. If $\left(G_{1}, P_{1}\right) \sim_{\Gamma}^{\mathrm{sc}}\left(G_{2}, P_{21}\right)$ and $P_{22} \sim_{\Gamma}^{\mathrm{sc}}$ o then $\left(G_{1}, P_{1}\right) \sim_{\Gamma}^{\mathrm{sc}}\left(G_{2}, P_{21} \| P_{22}\right)$

Proof. By any easy induction on the sum of the lengths of $P_{1}$ and $P_{2}$.

\subsection{Security proof}

Lemma 103 (SC Eval step local confinement). Suppose $t . c m d \neq$ skip and both high; $\Gamma \vdash^{\text {sc }} t$ and $(G, \mathcal{E}[t]) \longrightarrow{ }^{\text {eval }}$ $\left(G^{\prime}, P^{\prime}\right)$. Then $(G, \mathcal{E}[t]) \sim_{\Gamma}^{\mathrm{sc}}\left(G^{\prime}, P^{\prime}\right)$, and $P^{\prime}=t^{\prime} \| P_{0}^{\prime}$ where $\mathcal{E}[t] \sim_{\Gamma}^{\mathrm{sc}} t^{\prime}$.

Proof. Let $(\lambda, \mathcal{C})=\mathcal{E}$, define $t_{0}=\left.t \cup \lambda\right|_{\Gamma \text {, high }}$, and observe that by Lemma 93, $t_{0}$.ls.locks $\supseteq \lambda \cap t_{0}$.cmd.locks. Apply Lemma 33 to reduction $(G, \mathcal{E}[t])=\left(G, \mathcal{E}\left[n i l \mid t_{0}\right]\right) \longrightarrow^{\text {eval }}\left(G^{\prime}, P^{\prime}\right)$ to find $P^{\prime}=\mathcal{E}\left[t^{\prime}\right] \| P_{0}^{\prime}$ and $\left(G, t_{0}\right) \longrightarrow^{\text {eval }}\left(G^{\prime}, t^{\prime} \| P_{0}^{\prime}\right)$.

A low write, acquiring a low lock, or releasing a low lock would contradict t's high type. Therefore $G \sim \sim_{\Gamma}^{\text {wb }} G^{\prime}$.

We show that $P_{0}^{\prime} \sim_{\Gamma}^{\text {sc }} \mathcal{o}$. If the step was not by EC-Fork this is trivial. Otherwise $c=$ fork $c_{0}$ and $P_{0}^{\prime}=\left\langle L_{\oslash}, c_{0}\right\rangle \| \mathcal{o}$, and it remains to show that $h i g h ; \Gamma \vdash^{\mathrm{sc}} c_{0}$. This follows from inverting $t^{\prime}$ s typing derivation.

Finally we show that $\mathcal{E}[t]=\mathcal{E}\left[t_{0}\right] \sim_{\Gamma}^{\text {sc }} \mathcal{E}\left[t^{\prime}\right]$. Again, acquiring or releasing a low lock, or putting a low write in the write buffer, would contradict $t_{0}$ 's high type. Therefore $t_{0} . l s \sim_{\Gamma}^{\mathrm{sc}} t^{\prime}$.ls. From the type of $t$ is follows that high; $\Gamma \vdash^{\mathrm{sc}} t_{0}$, and preservation (Lemma 95) shows high; $\Gamma \vdash^{\mathrm{sc}} t^{\prime}$. Therefore $\mathcal{E}\left[t_{0}\right] \sim_{\Gamma}^{\mathrm{sc}} \mathcal{E}\left[t^{\prime}\right]$ holds.

Lemma 104 (SC Commit step confinement). Suppose high; $\Gamma \vdash^{\mathrm{sc}} t$ and $(G, t) \longrightarrow^{\text {commit }}\left(G^{\prime}, P^{\prime}\right)$. Then $(G, t) \sim_{\Gamma}^{\mathrm{sc}}\left(G^{\prime}, P^{\prime}\right)$.

Proof. Suppose a high value is committed; then the conclusion is immediate. Suppose instead a low value is committed, this contradicts they typing of $t$.

Lemma 105 (SC Global confinement). Suppose high; $\Gamma \vdash^{\mathrm{sc}} t$ and $(G, t) \longrightarrow^{o p}\left(G^{\prime}, P^{\prime}\right)$. Then $(G, t \| \mathrm{o}) \sim_{\Gamma}^{\mathrm{sc}}$ $\left(G^{\prime}, P^{\prime}\right)$.

Proof. Suppose $o p=$ commit. Conclude via Lemma 104. Instead suppose op $=$ eval and the step is not by EC-REAP. Inverting the step relation shows $t$ can be rewritten in form $\mathcal{E}[\langle L, c\rangle]$ where $c \neq$ skip and we conclude via Lemma 103. Finally suppose the step is by EC-REAP and conclude by observing $P^{\prime}=\mathrm{o} \sim \sim_{\Gamma}^{\mathrm{wb}} t \| \mathrm{o}$.

Definition 26 (SC-Quiet traces). Call trace $\mathcal{T}=\left(G_{1}, P_{1}\right),\left(G_{2}, P_{2}\right), \ldots,\left(G_{n}, P_{n}\right)$ sc-quiet in context $\Gamma$, written quiet ${ }_{\Gamma}^{\text {sc }} \mathcal{T}$, when for each pair of consecutive configurations, $\left(G_{i}, P_{i}\left\|t_{i}\right\| R_{i}\right)$ and $\left(G_{i+1}, P_{i}\left\|Q_{i+1}\right\| R_{i}\right)$ where $\left(G_{i}, t_{i}\right) \longrightarrow^{o p}\left(G_{i+1}, Q_{i+1}\right)$, one or more equivalences hold. First, $\left(G_{i}, t_{i} \| \mathrm{o}\right) \sim_{\Gamma}^{\text {sc }}\left(G_{i+1}, Q_{i+1}\right)$. Second, if $Q_{i+1}=t_{i+1} \| Q_{i+1}^{0}$ then $t_{i+1} \sim_{\Gamma}^{\mathrm{sc}} t_{i}$ and $Q_{i+1}^{0} \sim_{\Gamma}^{\mathrm{sc}} \mathrm{o}$.

Lemma 106. Suppose $\mathcal{T}::(G, t \| P) \Longrightarrow^{m *}\left(G^{\prime}, t^{\prime} \| P^{\prime}\right)$ where quiet $\Gamma_{\Gamma}^{\mathrm{sc}} \mathcal{T}$ and FrontReapFree $\mathcal{T}$. Then $t \sim_{\Gamma}^{\mathrm{sc}} t^{\prime}$ and $P \sim_{\Gamma}^{\mathrm{sc}} P^{\prime}$ and $G \sim_{\Gamma}^{\text {wb }} G^{\prime}$.

Proof. Proof by an easy induction on the length of $\mathcal{T}$.

Lemma 107. Suppose active $\mathcal{E}$ and wellStruct $t$ where synlocks $\left.t \subseteq t\right|_{\Gamma, \text { high }}$ and high $; \Gamma \vdash^{\mathrm{sc}} t$. Also suppose that $\left.t\right|_{\Gamma, \text { high }}$ and $\left.G\right|_{\Gamma, \text { high }}$ partition Lock $\left.\right|_{\Gamma, \text { high }}$. Then $\mathcal{T}::(G, \mathcal{E}[t] \|$ o $) \Longrightarrow{ }^{\text {sc* }}\left(G^{\prime}, \mathcal{E}\left[\left\langle L^{\prime}\right.\right.\right.$, skip $\left.\left.\rangle\right] \| P^{\prime}\right)$ where quiet $_{\Gamma}^{\mathrm{sc}} \mathcal{T}$ and FrontReapFree $\mathcal{T}$, and where $\left.L^{\prime}\right|_{\Gamma, \text { high }}$ and $\left.G^{\prime}\right|_{\Gamma, \text { high }}$ partition $\left.\mathbf{L o c k}\right|_{\Gamma, \text { high }}$ and where $\left.L^{\prime}\right|_{\Gamma, \text { high }}=$ $\left.\left.t\right|_{\Gamma, \text { high }} \backslash($ synlocks $t)\right|_{\Gamma, \text { high }}$ and $\left.P^{\prime}\right|_{\Gamma, \text { high }}=\emptyset$ and $L^{\prime} . w b=$ nil. Also hasEmptyWBs $\left(\mathcal{E}\left[\left\langle L^{\prime}\right.\right.\right.$, skip $\left.\left.\rangle\right] \| P^{\prime}\right)$. 
Proof. Let $\langle L, c\rangle=t$. Proof is by strong induction on size $c+$ size L.wb.

First suppose that $L . w b=(X:=i)+L_{0}$. Inverting the typing derivation shows $\Gamma(X)=$ high. Using a commit step and invoking the induction hypothesis yields a appropriate quiet $^{\mathrm{sc}}$, FrontReapFree trace with form,

$$
(G, t) \Longrightarrow^{\mathrm{sc}}\left(G[X \mapsto i],\left\langle L_{0}, c\right\rangle\right) \Longrightarrow^{\mathrm{sc} *}\left(G[X \mapsto i],\left\langle L^{\prime}, \mathbf{s k i p}\right\rangle\right) .
$$

Suppose instead that $L . w b=$ nil and proceed by case analysis on the typing derivation.

SC-LOAD: Here $c=x:=Y$ and $h i g h \sqsubseteq \Gamma(x)$. Construct a trace showing $(G, \mathcal{E}[t] \| \mathcal{o}) \Longrightarrow^{\text {tso }}(G,\langle L[x \mapsto i]$, skip $\rangle \| \mathcal{o})$. This trace is quiet ${ }^{\mathrm{sc}}$ because $\Gamma(x)=$ high typing ensures $L \sim_{\Gamma}^{\mathrm{wb}} L[x \mapsto i]$ and because high; $\Gamma \vdash^{\mathrm{sc}}$ skip. Conclude using the induction hypothesis, which is necessary to ensure that $w t=h i g h$ implies an empty output write buffer.

SC-STORE, SC-Eval: Similar to SC-LOAD. In the SC-Store case we must also commit a high write to ensure that the output state hasEmptyWBs.

SC-Sync: Here $c=\operatorname{sync} \ell$ do $c_{0}$ with $h i g h \sqsubseteq \Gamma(\ell)=h i g h$ and $h i g h ; \Gamma \vdash^{\text {sc }} c$. Recall $L . w b=n i l$ and

Suppose that $\ell \in L$. Reduce $\mathcal{E}[\langle L, c\rangle]$ to $\mathcal{E}\left[\left\langle L\right.\right.$, fence; $\left(c_{0}\right.$; fence $\left.\left.)\right\rangle\right]$ and conclude by invoking the induction hypothesis.

Suppose instead that $\ell \notin L$ then reduce $\mathcal{E}[\langle L, c\rangle]$ to $\mathcal{E}\left[\left\langle L \cup\{\ell\}\right.\right.$, holding $\ell$ do $\left.\left.c_{0}\right\rangle\right]$ and use the induction hypothesis to build a quiet $^{\text {sc }}$ and FrontReapFree derivation of

$$
(G, \mathcal{E}[t]) \Longrightarrow^{\mathrm{sc}}\left(G \backslash\{\ell\}, \mathcal{E}\left[\left\langle L \cup\{\ell\}, \text { holding } \ell \text { do } c_{0}\right\rangle\right]\right) \Longrightarrow^{\mathrm{sc*}}\left(G^{\prime}, \mathcal{E}\left[\left\langle L^{\prime}, \text { skip }\right\rangle\right] \| P^{\prime}\right) .
$$

By the induction hypothesis we have that $\left.L^{\prime}\right|_{\Gamma, \text { high }}=\left.\left.(L \cup\{\ell\})\right|_{\Gamma, \text { high }} \backslash\left(\right.$ synlocks holding $\ell$ do $\left.c_{0}\right)\right|_{\Gamma, \text { high }}$. From wellStruct $t$ it follows that synlocks $\left.c_{0}\right|_{\Gamma, \text { high }}=\emptyset$ and so $\left.L^{\prime}\right|_{\Gamma, \text { high }}=\left.L\right|_{\Gamma, \text { high }}$ as required.

SC-HoLD: Here $c=$ holding $\ell$ do $c_{0}$ with high $\sqsubseteq \Gamma(\ell)=$ high. As $\ell \in$ synlocks $\left.c \subseteq L\right|_{\Gamma \text {, high }}$, we can define $\mathcal{E}_{0}=(\lambda \cup\{\ell\}, \mathcal{C}[$ holding $\ell$ do $[\cdot]])$ where $(\lambda, \mathcal{C})=\mathcal{E}$ and active $\mathcal{E}^{\prime}$. Inverting the typing relation shows $w t=\Gamma(\ell)=$ high and high; high; $\Gamma \vdash^{\mathrm{wb}}\left\langle L, c_{0}\right\rangle$. By the induction hypothesis we have a quiet ${ }^{\mathrm{sc}}$ and FrontReapFree trace showing $\left(G, \mathcal{E}_{0}\left[\left\langle L, c_{0}\right\rangle\right]\right) \Longrightarrow{ }^{\text {sc* }}\left(G^{\prime}, \mathcal{E}_{0}\left[\left\langle L^{\prime}\right.\right.\right.$, skip $\left.\left.\rangle\right]\right)$ where $L^{\prime} . w b=$ nil. Because wellStruct $t$, it is not the case that $\ell \notin$ synlocks $c_{0}$, so $\ell \in L^{\prime}$. Finish by extending this trace using EC-HOLDRELEASE.

SC-Fence, SC-Fork: Similar to, but simpler than SC-Sync. Note that in the SC-Fork case the spawned thread has an empty write buffer and holds no locks.

SC-SEQ, SC-IF, SC-WHILE: Immediate by the induction hypothesis.

Lemma 108. Suppose active $\mathcal{E}$ and wellStruct $t$ and high; $\Gamma \vdash^{\mathrm{sc}} t$. Also suppose $\left.t\right|_{\Gamma, \text { high }}=\emptyset$ and $\left.G\right|_{\Gamma, \text { high }}=$ Lock $\left.\right|_{\Gamma, \text { high }}$. Then $\mathcal{T}::(G, \mathcal{E}[t] \| \mathcal{o}) \Longrightarrow^{\text {sc* }}\left(G^{\prime}, \mathcal{E}\left[\left\langle L^{\prime}\right.\right.\right.$, skip $\left.\left.\rangle\right] \| P^{\prime}\right)$ where quiet ${ }_{\Gamma}^{\text {sc }} \mathcal{T}$ and FrontReapFree $\mathcal{T}$, and where $\left.L^{\prime}\right|_{\Gamma, \text { high }}=\left.P^{\prime}\right|_{\Gamma, \text { high }}=\emptyset$ and $\left.G^{\prime}\right|_{\Gamma, \text { high }}=\left.\mathbf{L o c k}\right|_{\Gamma, \text { high }}$. Also hasEmptyWBs $\left(\mathcal{E}\left[\left\langle L^{\prime}\right.\right.\right.$, skip $\left.\left.\rangle\right] \| P^{\prime}\right)$.

Proof. Immediate using Lemma 107.

Lemma 109 (SC commit step security). Suppose the following hold.

$$
\left(G_{1}, t_{1}\right) \longrightarrow^{\text {commit }}\left(G_{1}^{\prime}, t_{1}^{\prime} \| \text { o }\right) \quad G_{1} \sim_{\Gamma}^{\text {sc }} G_{2} \quad t_{1} \sim_{\Gamma}^{\text {sc }} t_{2}
$$

Then $\left(G_{2}, t_{2}\right) \Longrightarrow{ }^{\mathrm{sc} *}\left(G_{2}^{\prime}, t_{2}^{\prime}\right)$ where $\left(G_{1}^{\prime}, t_{1}^{\prime}\right) \sim_{\Gamma}^{\mathrm{sc}}\left(G_{2}^{\prime}, t_{2}^{\prime}\right)$ and both $t_{2}^{\prime}$.locks $=t_{2}$.locks and $G_{2}^{\prime}$. locks $=$ $G_{2}$. locks.

Proof. Suppose that the $\longrightarrow{ }^{\text {commit }}$ operation commits write $X:=i$. Consider the case where $\Gamma(X)=h i g h$. We can conclude immediately, taking $t_{2}^{\prime}=t_{2}$ and $G_{2}^{\prime}=G_{2}$.

Suppose instead that $\Gamma(X)=$ low. By the definition of $\sim^{\text {sc }}$ we have that $L_{2} . w b=W_{21}+(X:=i)+W_{22}$ where for each $(Y:=j) \in W_{21}$, it is the case $\Gamma(Y)=$ high. (Although dynamic sc execution traces will never have more than one write buffer entry, we do not require a premise of this form.) Let $n$ denote the number of writes in $W_{21}$ and define $t_{2}^{\prime}$ and $G_{2}^{\prime}$ by taking $n+1$ commit steps. 
Lemma 110 (SC Eval step security). Suppose the following hold.

$$
\begin{array}{rrrr}
\left(G_{1}, t_{1}\right) \longrightarrow^{\text {eval }}\left(G_{1}^{\prime}, P_{1}^{\prime}\right) & p c ; \Gamma \vdash^{\mathrm{sc}} t_{1} \quad \text { wellStruct } t_{1} \quad t_{1} \cdot w b=\text { nil } \\
G_{1} \sim_{\Gamma}^{\mathrm{sc}} G_{2} & t_{1} \sim_{\Gamma}^{\mathrm{sc}} t_{2} & \left.t_{2}\right|_{\Gamma, \text { high }}=\emptyset & \left.G_{2}\right|_{\Gamma, \text { high }}=\left.\mathbf{\text { Lock }}\right|_{\Gamma, \text { high }}
\end{array}
$$

Then there exist $G_{2}^{\prime}$ and $P_{2}^{\prime}$ such that $\left(G_{1}^{\prime}, P_{1}^{\prime}\right) \sim_{\Gamma}^{\mathrm{wb}}\left(G_{2}^{\prime}, P_{2}^{\prime}\right)$ and $\left(G, t_{2}\right) \Longrightarrow{ }^{\mathrm{sc} *}\left(G_{2}^{\prime}, P_{2}^{\prime}\right)$. Furthermore $\left.P_{2}^{\prime}\right|_{\Gamma, \text { high }}=\emptyset$ and $\left.G_{2}^{\prime}\right|_{\Gamma, \text { high }}=\left.\mathbf{L o c k}\right|_{\Gamma, \text { high }}$.

Proof. We strengthen the induction hypothesis as follows. Whenever $P_{1}^{\prime}=t_{1}^{\prime} \| P_{10}^{\prime}$ there exist $\mathcal{T}, t_{2}^{\prime}$ and $P_{20}^{\prime}$ where $\mathcal{T}::\left(G_{2}, t_{2}\right) \Longrightarrow^{\text {tso* }}\left(G_{2}^{\prime}, P_{2}^{\prime}\right)$ and FrontReapFree $\mathcal{T}$ and $P_{2}^{\prime}=t_{2}^{\prime} \| P_{20}^{\prime}$ and where both $t_{1}^{\prime} \sim_{\Gamma}^{\text {wb }} t_{2}^{\prime}$ and $P_{10}^{\prime} \sim_{\Gamma}^{\text {wb }} P_{20}^{\prime}$. Proceed with strong induction on quantity $\left(\right.$ size $\left(t_{1} . c m d\right)+$ size $\left.\left(t_{2} . c m d\right)\right)$. Invert $t_{1} \sim_{\Gamma}^{\text {wb }} t_{1}$ using Lemma 85 to get three cases. Subcases of (i) will occasionally be completed by "falling through" to (ii).

(i) $t_{1}=\left\langle L_{1}, c\right\rangle$ and $t_{2}=\left\langle L_{2}, c\right\rangle$ where $L_{1} \sim_{\Gamma}^{\text {wb }} L_{2}$. Continue by inverting the $\longrightarrow^{\text {commit }}$ derivation.

EC-Store: Here $c=X:=y$. If $\Gamma(y)=$ low, the definition of $\sim_{\Gamma}^{\mathrm{wb}}$ shows $L_{1}(y)=L_{2}(y)$ and we conclude using Lemma 72. Otherwise $\Gamma(y)=h i g h$, inverting typing rule wB-Store gives $\Gamma(X)=$ high, and the result follows from Lemma 73.

EC-LOAD: Here $c=x:=Y$. If $\Gamma(x)=$ high we conclude as updating $L_{1}$ and $L_{2}$ is not observable. If instead $\Gamma(x)=$ low then typing ensures $\Gamma(Y)=$ low and equivalences $L_{1} \sim_{\Gamma}^{\mathrm{wb}} L_{2}$ and $G_{1} \sim_{\Gamma}^{\mathrm{wb}} G_{2}$, ensures we're writing identical values to $x$.

EC-EvalExP: Follows from Lemmas 70 and 71.

EC-SyncACQuire: Here $c=$ sync $\ell$ do $c_{0}$ and $\ell \in G_{1}$. First suppose that $\Gamma(\ell)=$ high. Let $\mathcal{E}=\left(\left.L_{1}\right|_{\Gamma, \text { low }},[\cdot]\right)$ and note that $t_{1}=\mathcal{E}\left[\left\langle\left. L_{1} \backslash L_{1}\right|_{\Gamma, \text { low }}, c\right\rangle\right]$. Inverting typing rule SC-SYNC shows that high; $\Gamma \vdash^{\mathrm{sc}} c_{0}$; using SC-SYNC, and noting that $\left.L_{1} \backslash L_{1}\right|_{\Gamma, \text { low }}$ has both an empty write buffer and no low locks, lets us find high; $\Gamma \vdash^{\mathrm{sc}}\left\langle\left. L_{1} \backslash L_{1}\right|_{\Gamma, \text { low }}, c\right\rangle$. Continue by falling through to case (ii).

Now suppose $\Gamma(\ell)=$ low. Here we will use that, from inversion, $G_{1}^{\prime}=G_{1} \backslash\{\ell\}$ and $L_{1}^{\prime}=L_{1} \cup\{\ell\}$. By the definition of $\sim^{\mathrm{sc}}$ and fact $L_{1} . w b=n i l$, we see that each write $(X:=i)$ in $L_{2} . w b$ has $\Gamma(X)=$ high. We can construct a derivation showing $\left(G_{2}, t_{2}\right) \Longrightarrow^{\text {sc* }}\left(G_{20}^{\prime},\left\langle L_{20}^{\prime}, c\right\rangle\right)$ where $\left(G_{2}, t_{2}\right) \sim_{\Gamma}^{\mathrm{sc}}\left(G_{20}^{\prime},\left\langle L_{20}^{\prime}, c\right\rangle\right)$, using finitely many $\longrightarrow^{\text {commit }}$ steps, each committing high variables . (Again, the premises of this lemma are weak, in that we don't assume $t_{2}$ 's write buffer contains at most one elements.) Applying the definition of $\sim^{\text {sc }}$ gives $\ell \in G_{20}^{\prime}$ and $L_{20}^{\prime} . w b=$ nil. Taking an EC-SYNCACQUIRE step gives derivation $\left(G_{2}, t_{2}\right) \Longrightarrow^{\text {sc* }}\left(G_{20}^{\prime} \backslash\{\ell\},\left\langle L_{20}^{\prime} \cup\{\ell\}, c_{0}\right\rangle\right)$. We take this result state to be $\left(G_{2}^{\prime}, P_{2}^{\prime}\right)$ and observe that $\left.P_{2}^{\prime}\right|_{\Gamma, \text { high }}=\left.\left(L_{20}^{\prime} \cup\{\ell\}\right)\right|_{\Gamma, \text { high }}=\left.\left(L_{2} \cup\{\ell\}\right)\right|_{\Gamma, \text { high }}=\emptyset$. It suffices to show $G_{20}^{\prime} \backslash\{\ell\} \sim_{\Gamma}^{\text {wb }} G_{1}^{\prime} \backslash\{\ell\}$, which follows from Lemmas 65 and 68 , and $L_{20}^{\prime} \cup\{\ell\} \sim_{\Gamma}^{\text {wb }}$ $L_{1}^{\prime} \cup\{\ell\}$, which follows from Lemmas 65 and 69 .

EC-Fence, eC-Fork, EC-HoldRelease: Similar to the EC-SyncAcquire case.

EC-SyncReEnteR: Here $c=$ sync $\ell$ do $c_{0}$. If $\Gamma(\ell)=$ high then using an argument to similar to the EC-SYNACQUiRE case fall through to (ii). If $\Gamma(\ell)=$ low then by $\sim^{\text {wb }} t_{1}$ and $t_{2}$ transition in lockstep and the case is trivial.

EC-HoldStep: Here $c=$ holding $\ell$ do $c_{0}$.

Suppose that $\Gamma(\ell)=h i g h$. As in case EC-SYNCACQUIRE we fall through to case (ii).

Let $t_{10}=\left\langle L_{1}, c_{0}\right\rangle$ and $t_{20}=\left\langle L_{2}, c_{0}\right\rangle$ as well as $\mathcal{E}=(\{\ell\}$, holding $\ell$ do $[\cdot])$. Inverting the evaluation relation gives $\ell \in L_{1}$ and $P_{1}^{\prime}=\mathcal{E}\left[t_{10}^{\prime}\right] \| P_{10}^{\prime}$ and $\left(G_{1}, t_{10}\right) \longrightarrow^{\text {eval }}\left(G_{1}^{\prime}, t_{10}^{\prime}\right)$. Applying the induction hypothesis to this eval step, using Lemma 92 to establish $p c ; \Gamma \vdash^{\mathrm{sc}} t_{10}$. This yields, among other properties,

$$
\mathcal{T}::\left(G_{2}, t_{20}\right) \Longrightarrow^{\mathrm{sc} *}\left(G_{2}^{\prime}, t_{20}^{\prime}\right)
$$

where FrontReapFree $\mathcal{T}$. Take $P_{2}^{\prime}$ to be $\mathcal{E}\left[t_{20}^{\prime}\right] \| P_{20}^{\prime}$ and finish by applying Lemma 100. .

eC-SeqStruct: Similar to EC-HoldStep.

EC-SEQSKIP: Immediate. 
EC-IfTRue: Here $c=$ if $b$ do $c_{t}$ else $c_{f}$ where $L_{1}[b] \Downarrow$ true and both $P_{1}^{\prime}=\left\langle L_{1}, c_{t}\right\rangle$ and $G_{1}^{\prime}=G_{1}$.

Suppose it's not the case that $\Gamma \vdash b$ : low. Then inverting the typing relation shows both $h i g h ; \Gamma \vdash^{\mathrm{sc}} c_{t}$ and $h i g h ; \Gamma \vdash^{\mathrm{sc}} c_{f}$. Without loss of generality assume $L_{2}[b] \Downarrow$ false and let $\left(G_{2}^{\prime}, P_{2}^{\prime}\right)=$ $\left(G_{2}^{\prime},\left\langle L_{2}, c_{f}\right\rangle\right)$. It suffices to show that $\left\langle L_{1}, c_{t}\right\rangle \sim_{\Gamma}^{\text {sc }}\left\langle L_{2}, c_{f}\right\rangle$. By Lemma 101 it suffices to show high; $\Gamma \vdash^{\text {wb }} L_{1}$.wb, which follows from hypothesis $L_{1} . w b=$ nil.

Suppose instead that that $\Gamma \vdash b:$ low. Lemma 84 shows $L_{2}[b] \Downarrow$ true so $\left(G_{2}, t_{2}\right) \Longrightarrow^{\text {sc* }}\left(G_{2},\left\langle L_{2}, c_{t}\right\rangle\right) \sim_{\Gamma}^{\text {wb }}$ $\left(G_{1},\left\langle L_{1}, c_{t}\right\rangle\right)=\left(G_{1}^{\prime}, P_{1}^{\prime}\right)$. Observe that this trace may begin with finitely many high commits.

eC-IfFalse, eC-WhileTrue, eC-WhileFalse: Similar to, or simpler than, EC-IFTrue. EC-REAP Trivial. .

(ii) $t_{1}=\mathcal{E}\left[\left\langle L_{1}, c_{1}\right\rangle\right]$ where $c_{1} \neq$ skip and $h i g h ; \Gamma \vdash^{\text {sc }}\left\langle L_{1}, c_{1}\right\rangle$ for some $w t_{1}$. By transitivity (Lemma 97) it suffices to show $\left(G_{1}^{\prime}, P_{1}^{\prime}\right) \sim_{\Gamma}^{\text {sc }}\left(G_{1}, t_{1}\right)$. Conclude via Lemma 103.

(iii) $t_{1}=\mathcal{E}\left[\left\langle L_{1}\right.\right.$, skip $\left.\rangle\right]$ and $t_{2}=\mathcal{E}\left[\left\langle L_{2}, c_{2}\right\rangle\right]$. We know the following for some $w t_{0}$.

$$
c_{2} \neq \text { skip } \quad L_{1} \sim_{\Gamma}^{\mathrm{wb}} L_{2} \quad \text { active } \mathcal{E} \quad \text { high; } \Gamma \vdash^{\mathrm{sc}}\left\langle L_{1}, c_{1}\right\rangle \quad \text { high } ; \Gamma \vdash^{\mathrm{sc}}\left\langle L_{2}, c_{2}\right\rangle
$$

By Lemma 108, we have $\mathcal{T}::\left(G, \mathcal{E}\left[\left\langle L_{2}, c_{2}\right\rangle\right] \| \mathcal{o}\right) \Longrightarrow^{\text {sc* }}\left(G_{2}^{\prime}, \mathcal{E}\left[\left\langle L_{2}^{\prime}\right.\right.\right.$, skip $\left.\left.\rangle\right] \| P_{2}^{\prime}\right)$ where quiet $\mathcal{T}_{\Gamma} \mathcal{T}$, FrontReapFree $\mathcal{T}$, and $\left.L_{2}^{\prime}\right|_{\Gamma, \text { high }}=\left.P_{2}^{\prime}\right|_{\Gamma, \text { high }}=\emptyset$. Furthermore hasEmpty $W B s\left(\mathcal{E}\left[\left\langle L_{2}^{\prime}\right.\right.\right.$, skip $\left.\left.\rangle\right] \| P_{2}^{\prime}\right)$ so hasEmptyWBs $\left(P_{2}^{\prime}\right)$. By Lemmas 65, 97 and 106 we find:

$$
\begin{gathered}
\mathcal{E}\left[\left\langle L_{2}^{\prime}, \text { skip }\right\rangle\right] \sim_{\Gamma}^{\mathrm{sc}} \mathcal{E}\left[\left\langle L_{2}, c_{2}\right\rangle\right] \sim_{\Gamma}^{\mathrm{sc}} t_{1} \\
G_{2}^{\prime} \sim_{\Gamma}^{\mathrm{wb}} G_{2} \sim_{\Gamma}^{\mathrm{wb}} G_{1} \\
P_{2}^{\prime} \sim_{\Gamma}^{\mathrm{sc}} \mathcal{O}
\end{gathered}
$$

Because $c_{2} \neq$ skip it is the case that size $\left(\mathcal{E}\left[\left\langle L_{2}^{\prime}\right.\right.\right.$, skip $\left.\left.\rangle\right] . c m d\right)<$ size $\left(\mathcal{E}\left[\left\langle L_{2}, c_{2}\right\rangle\right] . c m d\right)$, so we can use the induction hypothesis to find $G_{2}^{\prime \prime}$ and $P_{2}^{\prime \prime}$ such that $\left(G_{1}^{\prime}, P_{1}^{\prime}\right) \sim_{\Gamma}^{\text {wb }}\left(G_{2}^{\prime \prime}, P_{2}^{\prime \prime}\right)$ and $\left(G_{2}^{\prime}, \mathcal{E}\left[\left\langle L_{2}^{\prime}\right.\right.\right.$, skip $\left.\left.\rangle\right]\right) \Longrightarrow{ }^{\text {tso* }}$ By Lemma 102, $\left(G_{1}^{\prime}, P_{1}^{\prime}\right) \sim_{\Gamma}^{\text {wb }}\left(G_{2}^{\prime \prime}, P_{2}^{\prime \prime} \| P_{2}^{\prime}\right)$. Thus it suffices to show $\left.\left(P_{2}^{\prime \prime} \| P_{2}^{\prime}\right)\right|_{\Gamma, \text { high }}=\emptyset$, which is immediate, and $\left(G_{2}, t_{2}\right) \Longrightarrow{ }^{\text {tso* }}\left(G_{2}^{\prime \prime}, P_{2}^{\prime \prime} \| P_{2}^{\prime}\right)$, which is a consequence of Lemma 4.

Theorem 4 (SC Security). Suppose $\left(G_{1}, P_{1}\right) \sim_{\Gamma}^{\text {sc }}\left(G_{2}, P_{2}\right)$ and $\overline{p c} ; \Gamma \vdash^{\text {sc }} P_{1}$ and wellStruct $P_{1}$. Suppose also that $\left(G_{1}, P_{1}\right) \Longrightarrow^{\text {sc }}\left(G_{1}^{\prime}, P_{1}^{\prime}\right)$. Furthermore $\left.P_{2}\right|_{\Gamma, \text { high }}=\emptyset$ and $\left.G_{2}\right|_{\Gamma, \text { high }}=\left.\mathbf{L o c k}\right|_{\Gamma, \text { high }}$. Then there exists $G_{2}^{\prime}, P_{2}^{\prime}$ such that $\left(G_{1}^{\prime}, P_{1}^{\prime}\right) \sim_{\Gamma}^{\mathrm{sc}}\left(G_{2}^{\prime}, P_{2}^{\prime}\right)$ and $\left(G_{2}, P_{2}\right) \Longrightarrow{ }^{\text {sc* }}\left(G_{2}^{\prime}, P_{2}^{\prime}\right)$, and both $\left.P_{2}^{\prime}\right|_{\Gamma, \text { high }}=\emptyset$ and $\left.G_{2}^{\prime}\right|_{\Gamma, \text { high }}=\left.\mathbf{L o c k}\right|_{\Gamma, \text { high }}$.

Proof. Inverting the tso-evaluation relation and appealing to Lemma 98 gives

$$
\begin{aligned}
& P_{1}=P_{11}\left\|t_{1}\right\| P_{12} \\
& P_{2}=P_{21}\left\|P_{2}^{*}\right\| P_{22} \\
& P_{1}^{\prime}=P_{11}\left\|Q_{1}^{\prime}\right\| P_{12}
\end{aligned}
$$

where $P_{2}^{*}$ contains at most one thread (i.e., $P_{2}^{*} \in\left\{\mathfrak{o}, t_{2} \| \mathfrak{o}\right\}$ for some $t_{2}$ ) and the following hold:

$$
\begin{gathered}
\left(G, t_{1}\right) \longrightarrow{ }^{o p}\left(G_{1}^{\prime}, Q_{1}^{\prime}\right) \\
P_{11} \sim_{\Gamma}^{\mathrm{sc}} P_{21} \\
t \| \mathcal{o} \sim_{\Gamma}^{\mathrm{sc}} P_{2}^{*} \\
P_{12} \sim_{\Gamma}^{\mathrm{sc}} P_{22}
\end{gathered}
$$

It suffices to show that there exists $G_{2}^{\prime}$ and $Q_{2}^{\prime}$ such that $\left(G_{1}^{\prime}, Q_{1}^{\prime}\right) \sim_{\Gamma}^{\mathrm{sc}}\left(G_{2}^{\prime}, Q_{2}^{\prime}\right)$ and $\left(G_{2}, P_{2}^{*}\right) \Longrightarrow$ sc* $\left(G_{2}^{\prime}, Q_{2}^{\prime}\right)$. (Observe that while we could rename threads in $Q_{2}^{\prime}$, we do not need to; thread names are only really relevant for the data-race freedom argument.) Inspecting the definition of $\sim_{\Gamma}^{\mathrm{sc}}$ shows there are only three ways in which to find $t \| \mathrm{o} \sim_{\Gamma}^{\mathrm{sc}} P_{2}^{*}$. Proceed by case analysis.

First suppose that that the equivalence arises from Definition 25, clause $2 \mathrm{~b}$. Here $h i g h ; \Gamma \vdash^{\mathrm{sc}} t_{1}$ and via Lemma $105,\left(G_{1}, t \| \mathrm{o}\right) \sim_{\Gamma}^{\text {sc }}\left(G_{1}^{\prime}, Q_{1}^{\prime}\right)$. Conclude using Lemma 97 , which states $\sim^{\text {sc }}$ is an equivalence relation, and taking $G_{2}$ and $P_{2}^{*}$ as existential witnesses $G_{2}^{\prime}$ and $Q_{2}^{\prime}$. 
Second suppose that that the equivalence arises from definition 25, clause 2c. Here $P_{2}^{*}=t_{2} \| \mathcal{o}$ where $h i g h ; \Gamma \vdash^{\mathrm{sc}} t_{2}$ and $t_{1} \sim_{\Gamma}^{\mathrm{wb}} \mathrm{o}$. From $t_{1} \sim_{\Gamma}^{\mathrm{wb}} \mathrm{o}$ it follows that high; $\Gamma \vdash^{\mathrm{sc}} t_{1}$. Again taking $G_{2}$ and $P_{2}^{*}$ to be witnesses $G_{2}^{\prime}$ and $Q_{2}^{\prime}$ conclude with the following equational reasoning:

$$
\begin{array}{rlll}
\left(G^{\prime}, Q_{1}^{\prime}\right) & \sim_{\Gamma}^{\mathrm{sc}} & \left(G_{1}, t_{1} \| \mathrm{o}\right) & \text { by Lemma } 105 \\
& \sim_{\Gamma}^{\mathrm{sc}} & \left(G_{1}, \mathcal{o}\right) & \\
& \sim_{\Gamma}^{\mathrm{sc}} & \left(G_{2}, \mathcal{o}\right) & \text { by assumption } \\
& \sim_{\Gamma}^{\mathrm{sc}} & \left(G_{2}, t_{2} \| \mathrm{o}\right) & \\
& = & \left(G_{2}, P_{2}^{*}\right) &
\end{array}
$$

Third suppose that that the equivalence arises from Definition 25, clause $2 \mathrm{~d}$. Here $P_{2}^{*}=t_{2} \|$ o for some $t_{2}$ with $t_{1} \sim_{\Gamma}^{\text {sc }} t_{2}$. Finitely many inversions of the typing relation show $p c ; \Gamma \vdash^{\text {sc }} t_{1}$ for some $p c$. Similarly wellStruct $t_{1}$ and $\left.t_{2}\right|_{\Gamma, \text { high }}=\emptyset$. Suppose the step is a commit (that is, op $=$ commit); then conclude via Lemmas 109. If the step is an eval inverting the $\Longrightarrow^{\text {sc }}$ relation shows $t_{1} . w b=n i l$, and we conclude using Lemma 110.

Corollary 8. Suppose $\left(G_{1}, P_{1}\right) \sim_{\Gamma}^{\mathrm{sc}}\left(G_{2}, P_{2}\right)$ and $\overline{p c} ; \Gamma \vdash^{\mathrm{sc}} P_{1}$ and wellStruct $P_{1}$. Suppose also that $\left(G_{1}, P_{1}\right) \Longrightarrow^{\text {sc* }}\left(G_{1}^{\prime}, P_{1}^{\prime}\right)$. Furthermore $\left.P_{2}\right|_{\Gamma, \text { high }}=\emptyset$ and $\left.G_{2}\right|_{\Gamma, \text { high }}=\left.\mathbf{L o c k}\right|_{\Gamma, \text { high }}$. Then there exist $G_{2}^{\prime}$ and $P_{2}^{\prime}$ such that $\left(G_{1}^{\prime}, P_{1}^{\prime}\right) \sim_{\Gamma}^{\mathrm{sc}}\left(G_{2}^{\prime}, P_{2}^{\prime}\right)$ and $\left(G_{2}, P_{2}\right) \Longrightarrow^{\mathrm{sc*}}\left(G_{2}^{\prime}, P_{2}^{\prime}\right)$ and $\left.P_{2}^{\prime}\right|_{\Gamma, \text { high }}=\emptyset$.

Proof. By finitely many application of Theorem 4 and Lemmas 5 and 96.

Corollary 9. Suppose $G_{1} \sim_{\Gamma}^{\text {sc }} G_{2}$ and $p c ; \Gamma \vdash$ sc $c$ and src $c$. Also assume $\left.G_{2}\right|_{\Gamma, \text { high }}=\left.\mathbf{L o c k}\right|_{\Gamma, \text { high }}$. If $\left(G_{1},\left\langle L_{\oslash}, c\right\rangle\right) \Longrightarrow{ }^{\mathrm{sc*}}\left(G_{1}^{\prime}, \mathrm{o}\right)$ then $\left(G_{2},\left\langle L_{\oslash}, c\right\rangle\right) \Longrightarrow^{\mathrm{sc} *}\left(G_{2}^{\prime}, \mathrm{o}\right)$ for some $G_{2}^{\prime}$ where $G_{1}^{\prime} \sim_{\Gamma}^{\text {wb }} G_{2}^{\prime}$.

Proof. The first corollary to Theorem 4 shows $\left(G_{2}, t\right)$ evaluates to a configuration related to $\left(G_{1}^{\prime}, \mathbf{o}\right)$, and preservation (Lemma 96) and Lemmas 5, 9, and 108, show this evaluates to pool $o$.

Corollary 10 (SC Simple possibilistic noninterference). Suppose $p c ; \Gamma \vdash^{\text {sc }} c$ and src $c$. Then $c$ is possibilistically noninterfering under sc and $\Gamma$.

\section{Relating the type systems}

Lemma 111. If $p c ; \Gamma \vdash^{\text {tso }} c$ and src $c$ then there exists $w t$ such that $p c ; l o w ; \Gamma \vdash^{\text {wb }} c \Rightarrow w t$.

Proof. By induction on the derivation of $p c ; \Gamma \vdash^{\text {tso }} c$.

TSO-LOAD, TSO-Store, TSO-Eval Follows immediately since WB-LOAD, WB-Store, and wb-Eval respectively have the same premises.

TSO-SYNC Then $p c=$ low and $c$ has the form sync $\ell$ do $c^{\prime}$ and $\Gamma(\ell) ; \Gamma \vdash$ tso $c^{\prime}$. Since $p c=$ low we have $p c \sqsubseteq \Gamma(\ell)$ and $p c \sqsubseteq l o w$. By induction there exists $w t^{\prime}$ such that $\Gamma(\ell) ; l o w ; \Gamma \vdash^{\mathrm{wb}} c^{\prime} \Rightarrow w t^{\prime}$ and by Lemma 57 (iii) there exists $w t^{\prime \prime}$ such that $\Gamma(\ell) ;$ high; $\Gamma \vdash^{\text {wb }} c^{\prime} \Rightarrow w t^{\prime \prime}$. Then the result follows by WB-SYNC.

TSO-Hold Then $c$ has the form holding $\ell$ do $c^{\prime}$ contradicting the premise $\operatorname{src} c$.

TSO-FEnCE Then $p c=$ low so $p c \sqsubseteq$ low and the result follows by WB-Fence.

TSO-Fork Then $p c=l o w$ and $c$ has the form fork $c^{\prime}$ and $p c^{\prime} ; \Gamma \vdash{ }^{\text {tso }} c^{\prime}$. Since $p c=l o w$ we have $p c \sqsubseteq l o w$. By induction there exists $w t^{\prime}$ such that $p c^{\prime} ; l o w ; \Gamma \vdash^{\text {wb }} c^{\prime} \Rightarrow w t^{\prime}$ and by Lemma 57 (iii) there exists $w t^{\prime \prime}$ such that low; high; $\Gamma \vdash^{\mathrm{wb}} c^{\prime} \Rightarrow w t^{\prime \prime}$. Then the result follows by WB-Fork.

TSO-SEQ Then $c$ has the form $c_{1} ; c_{2}$ and $p c ; \Gamma \vdash^{\text {tso }} c_{1}$ and $p c ; \Gamma \vdash^{\text {tso }} c_{2}$. By induction there exist $w t_{1}$ and $w t_{2}$ such that $p c ; l o w ; \Gamma \vdash^{\mathrm{wb}} c_{1} \Rightarrow w t_{1}$ and $p c ; l o w ; \Gamma \vdash^{\mathrm{wb}} c_{2} \Rightarrow w t_{2}$. By Lemma 57 (iii) there exists $w t_{2}^{\prime}$ such that $p c ; w t_{1} ; \Gamma \vdash^{\mathrm{wb}} c_{2} \Rightarrow w t_{2}$, and the result follows by WB-SEQ.

TSO-IF Then $c$ has the form if $b$ do $c_{1}$ else $c_{2}$ and $\Gamma \vdash b: \tau$ and $p c \sqcup \tau ; \Gamma \vdash^{\text {tso }} c_{1}$ and $p c \sqcup \tau ; \Gamma \vdash^{\text {tso }} c_{2}$. By induction there exist $w t_{1}$ and $w t_{2}$ such that $p c \sqcup \tau ;$ low; $\Gamma \vdash^{\text {wb }} c_{1} \Rightarrow w t_{1}$ and $p c \sqcup \tau ;$ low; $\Gamma \vdash^{\text {wb }} c_{2} \Rightarrow$ $w t_{2}$, and the result follows by WB-IF. 
TSO-While Then $p c=l o w$ and $c$ has the form while $b$ do $c^{\prime}$ and $\Gamma \vdash b: l o w$ and $p c^{\prime} ; \Gamma \vdash^{\text {tso }} c^{\prime}$. By induction there exists $w t^{\prime}$ such that $p c^{\prime}$; low; $\Gamma \vdash^{\mathrm{wb}} c^{\prime} \Rightarrow w t^{\prime}$, and the result follows by WB-WHILE.

TSO-SKIP Then $c$ has the form skip and the result follows by WB-SKIP.

Lemma 112. If $p c ; w t ; \Gamma \vdash^{\mathrm{wb}} c \Rightarrow$ then $p c ; \Gamma \vdash^{\mathrm{sc}} c$.

Proof. by induction on the structure of the $\vdash^{\text {wb }}$ judgment.

wB-LoAd, wb-Store, wb-Eval wb-Fence, wb-Skip: Immediate as the corresponding SC-* rules have the same or fewer premises.

WB-Sync: Here $c=$ sync $\ell$ do $c_{0}$ and $p c \sqsubseteq \Gamma(\ell)$ by the induction hypothesis $\Gamma(\ell) ; \Gamma \vdash^{\text {sc }} c_{0}$. The result follows from SC-SYNC.

WB-Hold: Similar to WB-SynC.

WB-FORK: Here $c=$ fork $c_{0}$ and induction gives $p c ; \Gamma \vdash^{\mathrm{sc}} c_{0}$. The result follows from sC-Fork.

WB-SEQ: Here $c=c_{1} ; c_{2}$ and the induction hypothesis gives $p c ; \Gamma \vdash^{\mathrm{sc}} c_{1}$ and $p c ; \Gamma \vdash^{\mathrm{sc}} c_{2}$. The result follows from SC-SEQ.

WB-IF: Here $c=$ if $b$ do $c_{1}$ else $c_{2}$ where $\Gamma \vdash b: \tau$ and the induction hypothesis gives $p c \sqcup \tau ; \Gamma \vdash \vdash^{\text {sc }} c_{1}$ and $p c \sqcup \tau ; \Gamma \vdash^{\mathrm{sc}} c_{2}$. Conclude with rule SC-IF.

WB-WhILE: Here $c=$ while $b$ do $c_{0}$. Inverting the typing relation shows $p c=$ low and $\Gamma \vdash b:$ low. Furthermore, for some $p c_{0}$ and $u t_{0}$, it is the case that $p c_{0} ; u t ; \Gamma \vdash^{\mathrm{wb}} c_{0} \Rightarrow u t_{0}$. By induction $p c_{0} ; \Gamma \vdash^{\mathrm{sc}}$ $c_{0}$. Conclude with rule SC-WHiLE.

\section{References}

Geoffrey Smith and Dennis Volpano. Secure information flow in a multi-threaded imperative language. In Proc. 25th ACM Symp. on Principles of Programming Languages (POPL), pages 355-364, San Diego, California, January 1998.

Jefrey A. Vaughan and Todd Millstein. Secure information flow for concurrent programs under total store order. In CSF '12, 2012. 